\title{
Pleiotropy and epistasis within and between signaling pathways defines the genetic architecture of fungal virulence
}

\author{
Cullen Roth ${ }^{1,2}$, Debra Murray ${ }^{1}$, Alexandria Scott ${ }^{1}, \mathrm{Ci} \mathrm{Fu}^{3}$, Anna F. Averette ${ }^{3}$, Sheng Sun ${ }^{3}$, \\ Joseph Heitman ${ }^{3}$, and Paul M. Magwene ${ }^{1^{*}}$
}

${ }^{1}$ Department of Biology, Duke University, Durham, North Carolina, United States of America

2 University Program in Genetics and Genomics, Duke University, Durham, North Carolina, United States of America

${ }^{3}$ Department of Molecular Genetics and Microbiology, Duke University Medical Center, Durham, North Carolina, United States of America

* Corresponding author

E-mail: paul.magwene@duke.edu (PM) 


\section{Abstract}

Cryptococcal disease is estimated to affect nearly a quarter of a million people annually. Envi- 2 ronmental isolates of Cryptococcus deneoformans, which make up 15 to $30 \%$ of clinical infections 3 in temperate climates such as Europe, vary in their pathogenicity, ranging from benign to hyper- 4 virulent. Key traits that contribute to virulence, such as the production of the pigment melanin, an 5 extracellular polysaccharide capsule, and the ability to grow at human body temperature have been 6 identified, yet little is known about the genetic basis of variation in such traits. Here we investigate 7 the genetic basis of melanization, capsule size, thermal tolerance, oxidative stress resistance, and antifungal drug sensitivity using quantitative trait locus (QTL) mapping in progeny derived from a cross between two divergent $C$. deneoformans strains. Using a "function-valued" QTL analysis 10 framework that exploits both time-series information and growth differences across multiple envi- ${ }_{11}$ ronments, we identified QTL for each of these virulence traits and drug susceptibility. For three 12 QTL we identified the underlying genes and nucleotide differences that govern variation in virulence $\quad 13$ traits. One of these genes, RIC8, which encodes a regulator of CAMP-PKA signaling, contributes 14 to variation in four virulence traits: melanization, capsule size, thermal tolerance, and resistance to 15 oxidative stress. Two major effect QTL for amphotericin B resistance map to the genes SSK1 and 16 SSK2, which encode key components of the HOG pathway, a fungal-specific signal transduction $\quad{ }_{17}$ network that orchestrates cellular responses to osmotic and other stresses. We also discovered 18 complex epistatic interactions within and between genes in the HOG and cAMP-PKA pathways that 19 regulate antifungal drug resistance and resistance to oxidative stress. Our findings advance the 20 understanding of virulence traits among diverse lineages of Cryptococcus, and highlight the role of ${ }_{21}$ genetic variation in key stress-responsive signaling pathways as a major contributor to phenotypic 22 variation.

\section{Author summary}

Different environmental isolates (strains) of the same microbial species can vary greatly in 25 their ability to cause disease, ranging from avirulent to hypervirulent. What makes some strains 26 deadly pathogens, while others are relatively benign? This study describes the characterization 27 
of key genetic differences that underlie variation in traits thought to promote virulence in Cryp- 28 tococcus deneoformans, a wide-spread opportunistic fungal pathogen. Using a combination of 29 quantitative genetic and molecular genetic approaches we dissected the genetic architecture of 30 virulence-related cellular traits (melanin production and the production of a polysaccharide cap- 31 sule), physiological responses to stress (tolerance of thermal, oxidative, and osmotic stress), and 32 sensitivity to multiple antifungal drugs. Strikingly we find that variation in most of these traits is gov- 33 erned by a small number of genetic differences that modify the function of two major cell signaling 34 networks, cyclic AMP-Protein Kinase A (cAMP-PKA) signaling and a fungal specific MAP-kinase 35 cascade called the high osmolarity glycerol (HOG) pathway. Similar to recent studies in a number 36 of other fungal species, our findings point to an outsize role for a small number of highly pleiotropic $\quad 37$ signaling pathways in potentiating phenotypic variation both within and between fungal species. 38

\section{Introduction}

Over the last two decades, fungal species have emerged as major threats and pathogens [1, 2], 40 affecting endangered plant and animal species [3-7], reducing crop yields [8, 9], and causing hu- 41 man illness [10-13]. The propensity of fungal pathogens to cause disease is a complex outcome 42 dependent on a variety of underlying physiological features that facilitate survival in stressful host ${ }_{43}$ niches, such as the ability to forage and acquire nutrients [14], to tolerate bombardment from reac- $\quad 44$ tive oxygen species [15], and to mount a successful defense against (or evade) the host immune 45 system $[16,17]$. Significant progress has been made with respect to understanding the cell and 46 molecular biology of fungal pathogenesis; numerous virulence-related traits and key genes and 47 pathways that regulate these traits have been identified for many fungal pathogens [18-29]. Sim- 48 ilarly, the availability of low-cost, high-throughput genome sequencing has greatly advanced the 49 understanding of genetic variation and population structure for many fungal pathogens [30-36]. 50 However, despite advances in both the molecular genetics of fungal pathogenesis and the ge- ${ }_{51}$ nomics of pathogenic species, for most fungal pathogens we have a limited understanding of the 52 genetic changes between isolates that contribute to differences in virulence traits [37, 38].

Basidiomycete fungi of the genus Cryptococcus are important human pathogens, estimated to 54 affect nearly a quarter of a million people worldwide annually [39]. The majority of cryptococcal 55 
infections occur in individuals with compromised or suppressed immune systems, such as those 56 combating AIDS/HIV or organ transplant recipients, however infections in seemingly healthy peo- $\quad 57$ ple have also been reported [40-44]. If untreated, cryptococcal meningitis is uniformly fatal, and 58 current estimates of mortality rates for individuals receiving treatment for cryptococcosis vary by 59 region, from $10-30 \%$ in North America to as high as $50-70 \%$ in parts of sub-Saharan Africa 60 $[39,45,46]$. Most cases of cryptococcosis are due to infections of Cryptococcus neoformans, but the sister species Cryptococcus deneoformans (formerly referred to as C. neoformans var. neofor- 62 mans serotype $\mathrm{D}$ ) is responsible for a significant number of clinical cases in temperate regions of the world, and mixed infections of both $C$. neoformans and $C$. deneoformans have been reported [47-50]. In addition to their clinical relevance, Cryptococcus species are attractive model organisms for studying traits associated with virulence due to their experimental tractability, including a well characterized sexual cycle featuring recombination and vegetative growth as haploid yeasts $[19,51,52]$, methods for transformation and genetic engineering, [53-56], and a large panel of gene deletion strains [21].

Key Cryptococcus virulence traits include melanization, resistance to oxidative stress, formation of an extracellular capsule, and thermal tolerance [57-63]. Cryptococcus species are opportunistic rather than obligate pathogens, and not all Cryptococcus species or strains exhibit the full complement of traits that are thought to be required for pathogenesis in animal hosts [64]. Furthermore, many of these traits, such as the production of melanin and the polysaccharide capsule, 74 are likely to impose a significant metabolic cost. Given this, there has been considerable interest 75 in the selective forces that have contributed to the origin and maintenance of virulence traits. The 76 "accidental pathogen hypothesis" suggests that these traits evolved due to interactions with micro- 77 bial predators and physiological stresses within non-pathogenic niches; thus virulence-associated 78 traits are likely to have dual roles in the natural environment and within animal hosts [1,65-67]. 79 For example, the biosynthesis of melanin, a hydrophobic high-molecular weight black or brown 80 pigmented polymer, buffers Cryptococcus cells from thermal stress and protects cells against so- $\quad{ }_{81}$ lar radiation $[68,69]$. Within the host niche, this pigment prevents damage from reactive oxygen 82 species $[28,57,70]$. The extracellular polysaccharide capsule is thought to protect cells from be- 83 ing phagocytosed by amoeboid protozoans, natural predators of Cryptococcus [71]. In the host 84 environment, the capsule protects cells from the host immune response, including phagocytosis 85 
by macrophages and oxidative stress, and shed capsule material has a variety of activities on host immune cells $[63,72,73]$. Thermal tolerance, which could be selected for by extreme seasonal temperatures, allows for the infection of mammalian and avian hosts with high body temperatures [74-77]. Cryptococcus species most often associated with human disease, C. neoformans, C. deneoformans, and C. gattii display the highest thermal tolerance [64].

Another important trait, resistance to antifungal drugs, is not considered a virulence trait per se, because it is not necessary for establishing an initial infection within a host. However, antifungal 92 resistance can lead to recurring disease and is thus a clinically relevant trait [62, 78, 79]. Ampho- 93 tericin $B$ is one of the few drugs effective in the treatment of cryptococcosis $[80,81]$, killing fungal 94 cells by binding to and sequestering ergosterol from the bilipid membrane $[82,83]$. While resis- 95 tance to amphotericin B is rare [84], a recent examination of clinical isolates observed an increase 96 in the amphotericin B minimum inhibitory concentrations compared to inhibitory concentrations 97 taken from the same region of study ten years earlier [85]. Globally, there are an increasing num- 98 ber of reports documenting rises in antifungal resistance $[86,87]$ and understanding the genetic 99 architecture of drug susceptibility is integral to combating this growing trend.

There is considerable variation in virulence-associated traits and antifungal drug resistance both within and between Cryptococcus species [88-97]. One of the most powerful approaches for 102 dissecting the genetic basis of phenotypic variation is quantitative trait locus (QTL) mapping [98, 103 99]. QTL mapping has been employed extensively in the model yeast Saccharomyces cerevisiae 104 [100-106] and has been used to explore the genetic basis of virulence-related traits for a number of 105 fungal plant pathogens [107-109]. However, there have been relatively few QTL studies in human 106 fungal pathogens - one in Aspergillus [110] and two previous QTL studies in Cryptococcus [111, 107 112]. In these important pathogens, QTL mapping has the potential to enhance our understanding 108 of the genetic basis of virulence.

A common, though not universal, characteristic of many microbial QTL mapping studies is the ${ }_{110}$ use of microbial growth as a proxy for physiological responses to different environmental conditions ${ }_{111}$ or stresses [105]. However, the use of growth as a trait presents some important challenges. For 112 example, two strains may exhibit drastically different lag times and exponential growth rates yet still ${ }_{113}$ reach the same final population density. Furthermore, classic mathematical models of microbial ${ }_{114}$ growth, such as the Gompertz equation or logistic growth models [113], often fail to capture the 115 
real world complexities of microbial growth [114]. Generally speaking, the population density of a 116 microbial culture is a complex function dependent upon both time and the magnitude of exposure ${ }_{117}$ to a stress or environment. Such data are often termed longitudinal or function valued and a body 118 of statistical methods have been developed for function-valued data in which the order and spacing 119 of data is retained [115-121]. Studies which use frameworks for the analysis of function-valued 120 data can be found across the fields of biology including ecology, developmental biology, and crop ${ }_{121}$ genetics [122-129]. With respect to QTL mapping, function-valued methods have been shown to 122 increase the ability to detect QTL $[122,124,130-136]$.

Here we describe the genetic architecture of six clinically important and complex phenotypic $\quad 124$ traits in C. deneoformans: melanization, capsule size, thermal tolerance, growth under oxidative 125 stress, and resistance to the antifungal drugs amphotericin B and fludioxonil. Based on a mapping 126 population derived from a cross between a laboratory strain (XL280; [137]) and an environmental ${ }_{127}$ isolate (431 $\alpha$; [138]), we employed genome-wide sequencing and function-valued QTL mapping 128 to identify genetic differences that underlie variation in each of the above traits. We discovered a 129 major QTL with highly pleiotropic effects on melanization, capsule size, high temperature growth, 130 and resistance to oxidative stress. We identified a likely causal variant for this shared QTL, a 131 premature stop codon in the gene RIC8, a component of the cyclic AMP-protein kinase A (cAMP- ${ }_{132}$ PKA) signaling pathway. Interestingly, allelic variation at RIC8 has antagonistic effects with regard 133 to virulence potential, increasing tolerance to high temperatures while decreasing melanization. 134 We also identified two QTL that underlie amphotericin B susceptibility, and mapped the likely causal $\quad 135$ variants to the genes SSK1 and SSK2, components of the high-osmolarity glycerol (HOG) pathway. ${ }_{136}$ Epistatic interactions within the HOG pathway, and between the HOG and CAMP-PKA pathways, 137 also contribute to variability in drug resistance, thermal tolerance, and oxidative stress resistance. ${ }_{138}$ This study highlights the importance of genetic variation in key signal transduction pathways that 139 regulate stress responses in Cryptococcus and other fungi, and illustrates the complex effects that 140 such variants may have with respect to virulence potential. 


\section{Results}

\section{A high resolution genetic mapping population}

We generated an $F_{1}$ mapping population by crossing the $C$. deneoformans strains XL280a ${ }_{144}$ and XL280 $\alpha$ SS $[92,137]$ with the environmental isolate, $431 \alpha[92,139,140]$ in $\alpha-\alpha$ unisexual and 145 a- $\alpha$ bisexual matings [92]. The haploid genomes of the parental strains and 101 segregants were 146 sequenced at approximately $64 \times$ coverage. Following filtering, 92,103 sites were identified that 147 differ between the parental strains, and genotypes at each of these variable sites were called for ${ }_{148}$ each segregant based on mapping to the $\mathrm{XL280} \alpha$ reference genome [137]. Variable sites were 149 collapsed into unique haploblocks based on genetic exchange events, resulting in 3,108 unique 150 haploblocks. The average size of the haploblocks was $5.4 \mathrm{~kb}$ (approximately $1 \mathrm{cM}$; [141]) with a 151 maximum and minimum size of 6.3 and $4.4 \mathrm{~kb}$, respectively (S1 Fig). This set of 101 segregants, $\quad 152$ parental strains, and their genotypic states at each of the 3,108 haploblocks served as the mapping 153 population for subsequent QTL analyses.

\section{QTL for melanization}

Melanization is an important phenotype related to virulence, and a previous study that uti- ${ }_{156}$ lized the same set of progeny used here observed significant variation in melanin production [92]. 157 Melanization was quantified from scanned images of colonies grown on L-DOPA plates by calcu- ${ }_{158}$ lating the mean grayscale intensity (the amount of light reflected off of a colony) of each segre- 159 gant. The parental strains differ in their production of melanin - the XL280a parental strain has 160 an opaque, beige appearance and the $431 \alpha$ parental strain grew as a dark brown colony (Fig 1A). ${ }_{161}$ There is significant variation in the production of melanin among the segregants, with most progeny 162 exhibiting melanization intermediate between the two parental phenotypes (Fig 1A). Less than 8\% ${ }_{163}$ of progeny exhibited transgressive melanin phenotypes that were more pigmented than the $431 \alpha \quad 164$ parental strain or lighter than the XL280a parental strain. QTL mapping of the melanin phenotype ${ }_{165}$ identified a single large peak on chromosome 14 (Fig 1B). Segregants with the XL280a genotype ${ }_{166}$ at this locus had lighter colonies (higher mean intensity), while segregants with the $431 \alpha$ allele ${ }_{167}$ produced darker colonies (lower mean intensity, Fig 1C and 1D). Based on the regression model ${ }_{168}$ 


\section{QTL for variation in capsule diameter}

The production of a polysaccharide capsule is another well-studied virulence trait in Crypto- ${ }^{171}$ coccus. India ink stained cells from each segregant were imaged using brightfield microscopy, 172 and cell body and capsule diameter were measured. There was a strong allometric relationship ${ }_{173}$ between capsule diameter and cell size (Fig 2A). To account for this "size effect" we regressed 174 capsule diameter on the combined cell and capsule diameter, and used the residuals from this 175 relationship as a measure of size-standardized capsule size (Fig 2B). A similar model, comparing 176 cell diameter to the cell and capsule diameter was also calculated (S2 Fig).

QTL mapping of the standardized capsule size identified a single significant peak on chromo- ${ }_{178}$ some 14 (Fig 2C). Heritablity at this locus was estimated to be $17 \%$. At the peak of this QTL, 179 segregants with the XL280a genotype had larger (positive) capsule diameter residuals compared 180 to sibling strains with the $431 \alpha$ allele (Fig 2B).

\section{Negative transgressive segregation in temperature and amphotericin B tolerance}

Microbial stress responses are dependent on both the intensity of exposure and time since 183 exposure. In order to capture both aspects of such responses to thermal stress and antifungal ${ }_{184}$ drugs we employed an automated phenotyping framework to measure microbial growth over time 185 across multiple environmental conditions. For each of the segregants and parental strains, growth ${ }_{186}$ in liquid media was measured on an absorbance microplate reader for a total of eleven experimen- ${ }_{187}$ tal conditions consisting of combinations of temperature $\left(30,37\right.$, and $\left.39^{\circ} \mathrm{C}\right)$ and amphotericin $\mathrm{B} \quad{ }_{188}$ (concentrations of $0,0.075,0.125$, and $0.175 \mu \mathrm{g} / \mathrm{ml}$ ). These conditions were chosen to maximize ${ }_{189}$ the phenotypic variation within the mapping population. In each experimental condition, the opti- 190 cal density $\left(\mathrm{OD}_{595 \mathrm{~nm}}\right)$ was measured at 15-minute intervals for 72 hours. Each set of time series 191 measurements was treated as a growth curve and four replicate growth curves were measured per 192 segregant. After normalization and base-lining, total growth was estimated as the area under each 193 growth curve. Fig 3 represents the median growth curve across replicates for each segregant at 194 each combination of temperature and amphotericin B concentration. 
A

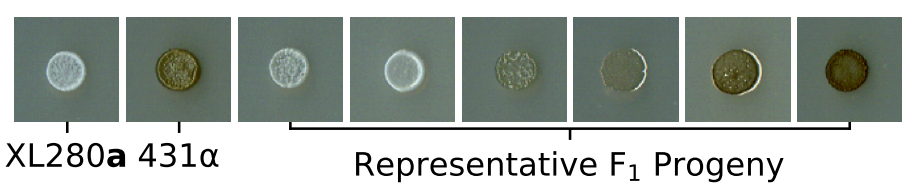

B
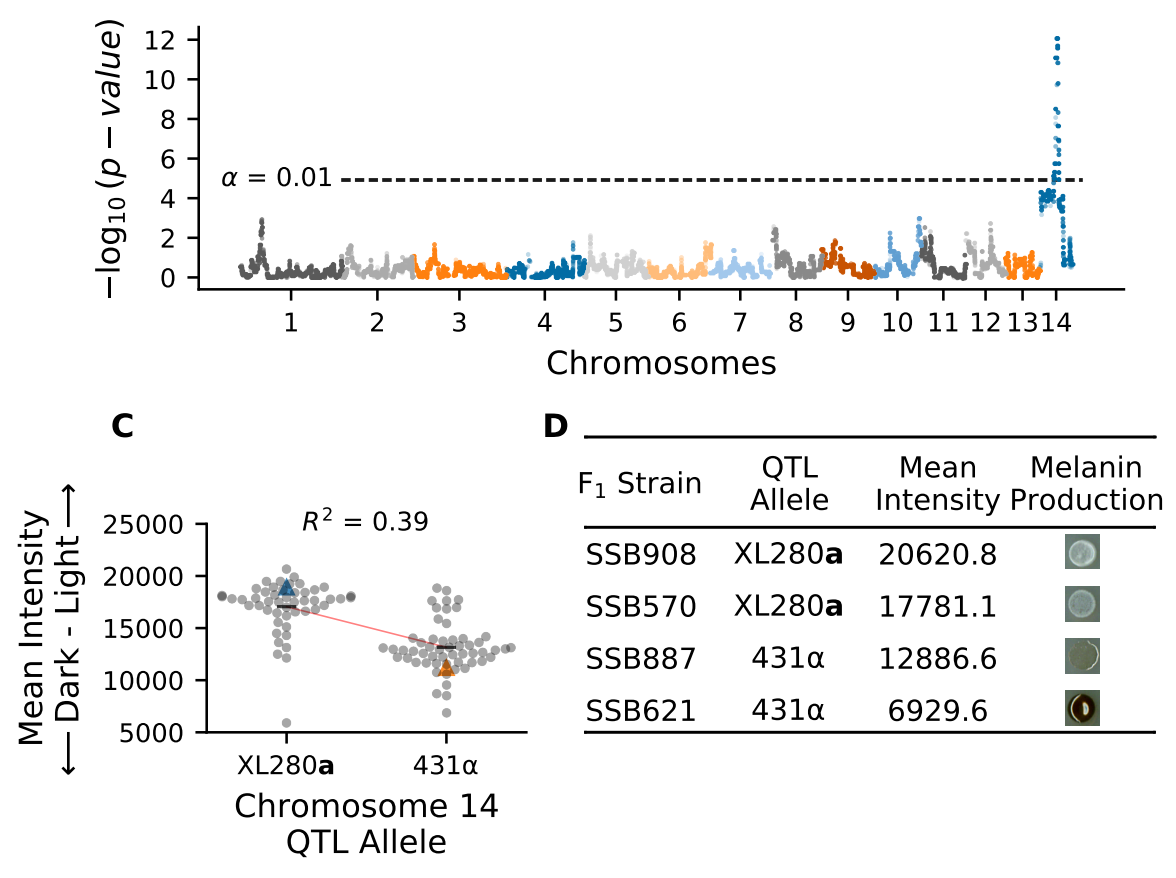

Fig 1. QTL analysis of variation in melanin production. A) Melanization phenotypes of parental strains - XL280a and $431 \alpha$ - and examplar phenotypes of their segregants. B) Manhattan plot of the association between genotype and melanin production. The x-axis represents chromosomal locations of haploblocks and the y-axis represents the strength of association between genotype and variation in melanization. The significance threshold (dashed horizontal line) was determined via permutation. C) Mean grayscale intensity (y-axis; arbitrary units) of segregants (gray dots) grown on L-DOPA plates as a function of genotype (x-axis) at the QTL peak on chromosome 14. Blue and orange triangles mark the parental phenotypes while black horizontal lines denote the phenotypic means by allele. The red line represents a regression model relating phenotype to genotype; this regression model explains $39 \%$ of the variation in melanin production. D) QTL allele, mean intensity, and melanin production of example $F_{1}$ strains. 

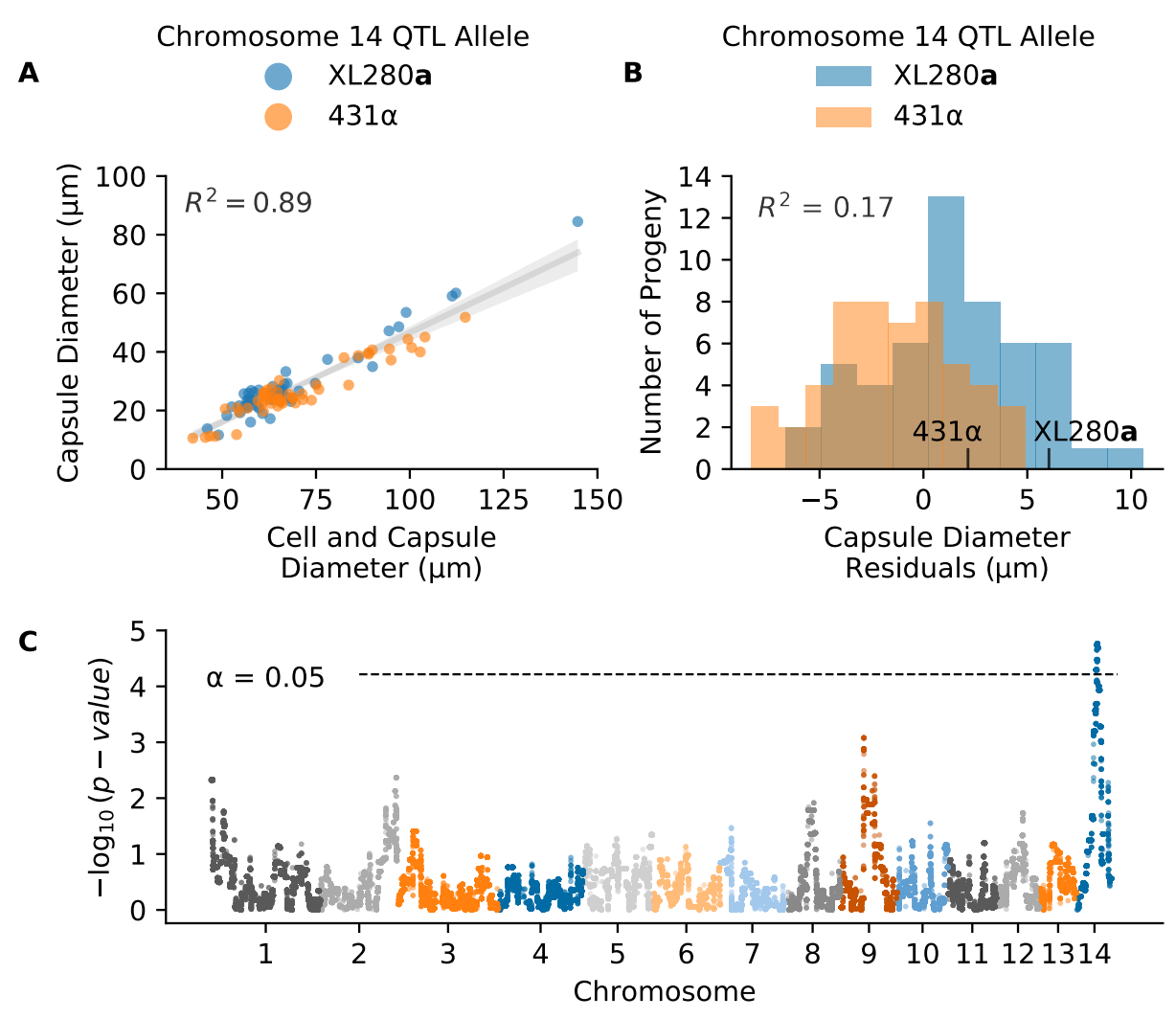

Fig 2. QTL analysis of variation in capsule diameter residuals. A) Measurements of the average cell and capsule diameters ( $\mathrm{x}$-axis) and the calculated capsule diameter ( $\mathrm{y}$-axis) per segregant colored by the chromosome 14 QTL allele in C. B) Histogram of capsule diameter residuals calculated from the linear regression model in A, separated by chromosome 14 QTL allele. C) Manhattan plot of the association between genotype and capsule diameter residuals. The $x$-axis represents chromosomal locations of haploblocks and the $y$-axis represents the strength of association between genotype and variation in capsule diameter residuals.

There was significant variation in the growth trajectories across the eleven temperature by 196 amphotericin $\mathrm{B}$ conditions. At the permissive conditions of $30^{\circ} \mathrm{C}$ and no amphotericin $\mathrm{B}$, most of ${ }_{197}$ the segregants growth curves fell near or between the parental growth curves. Conversely, at 198 $37^{\circ} \mathrm{C}$ without amphotericin B, the parental strain $431 \alpha$ outgrew the other parental strain, XL280a, 199 as well as all of the segregants. In this high temperature condition $35 \%$ of segregants outgrew 200 


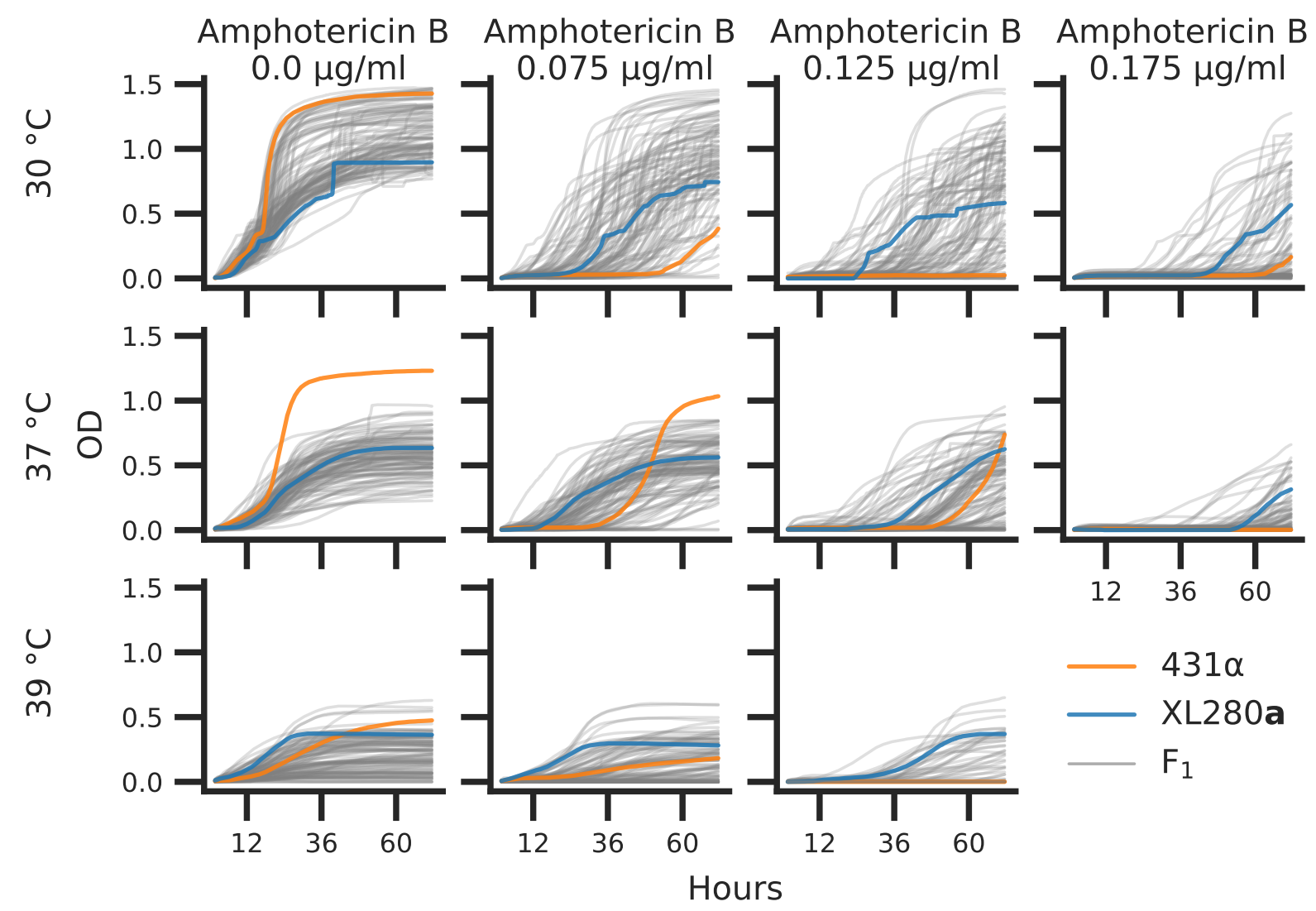

Fig 3. C. deneoformans growth curves. Parental strains and progeny were assayed for growth across combinations of temperatures (rows) $30^{\circ}, 37^{\circ}, 39^{\circ} \mathrm{C}$ and concentrations of amphotericin $\mathrm{B}$ (columns) at $0.0,0.075,0.125$, and $0.175 \mu \mathrm{g} / \mathrm{ml}$. Optical density $\left(\mathrm{OD}_{595 \mathrm{~nm}}, \mathrm{y}\right.$-axis) was measured every 15 minutes for 72 hours (x-axis). The median optical density across replicates is shown for the parental strains, XL280a (blue curve) and $431 \alpha$ (orange curve), and the $F_{1}$ segregants (grey curves).

the XL280a parental strain. In most other combinations of temperature and amphotericin B stress, 201 $F_{1}$ progeny displayed negative transgressive segregation, with less total growth compared to the 202 parental strains.

At $30^{\circ} \mathrm{C}$, across amphotericin B concentrations of $0.075,0.125$ and $0.175 \mu \mathrm{g} / \mathrm{ml}$, the $431 \alpha \quad 204$ progenitor strain grew poorly and across these experimental conditions only 33, 24, and 19\% of 205 segregants (respectively) outgrew the XL280a parental strain. Surprisingly, 431 $\alpha$, when exposed to 206 a combination of modest thermal stress $\left(37^{\circ} \mathrm{C}\right)$ and moderate amphotericin B concentrations $(0.075207$ and $0.125 \mu \mathrm{g} / \mathrm{ml}$ ), grows better than when exposed to drug stress alone. At $37^{\circ} \mathrm{C}$ in conditions 208 of $0.075,0.125$ and $0.175 \mu \mathrm{g} / \mathrm{ml}$ of amphotericin $\mathrm{B}, 44,24$, and $18 \%$ of segregants outgrow the 209 $\mathrm{XL280}$ a progenitor strain, respectively. At $39^{\circ} \mathrm{C}$ the parental strains had similar total growth with the 210 
$431 \alpha$ displaying a greater final OD. Across amphotericin B conditions at $39^{\circ} \mathrm{C}$, the $\mathrm{XL} 280$ a parent ${ }^{211}$ outgrew the $431 \alpha$ strain, and only a modest number of offspring $(\sim 7 \%)$ outgrew either parental 212 strain. Taken as a whole, these data revealed a temporally dynamic and varying response to 213 temperature and antifungal stress.

\section{Dynamic QTL underlying temperature stress and resistance to amphotericin B}

A common approach to identify QTL associated with variation in microbial growth is to map the ${ }_{216}$ maximum growth rate or the population density at a specific time point and regress this value across ${ }_{217}$ variable genetic loci. This approach however fails to capture genotype-phenotype associations 218 that change across time. Time varying traits are often referred to as function-valued [120]. Here, a 219 function-valued, marker-regression approach was employed to quantify the relationship between 220 genotype and growth phenotypes at each variable haploblock across the 72-hour time courses of ${ }_{221}$ each temperature and amphotericin B combination.

Temporally dependent QTL underlying variation across each of the eleven experimental com- ${ }^{223}$ binations of temperature and concentrations of amphotericin B were identified with a temporal ${ }_{224}$ regression model. Following model fitting, the $-\log _{10} p$-values (effect of a potential QTL) were ${ }_{225}$ calculated across time points (S3 Fig), and significance thresholds were estimated by permutation ${ }_{226}$ tests [142]. For nine of the eleven conditions, between one and three QTL (on different chromo- ${ }_{227}$ somes) were identified across the time course (S4 Fig). Across the combinations of temperature 228 and amphotericin B stress, taking the maximum association at each variable site across the 72- ${ }^{229}$ hour time course (Fig 4), a total of thirteen QTL above the thresholds of significance were identified 230 across the eleven temperature and amphotericin B conditions (S5 Fig). Nearly all of the QTL iden- ${ }^{231}$ tified showed temporally dependent behavior, with early time-series associations for some QTL 232 and later associations for others (Fig 5A). Taking the maximum association across combinations 233 of temperature and amphotericin B stress, and across time, four unique QTL on chromosomes 2, 234 11, 12, and 14 were identified (Fig 5B). Two of these QTL, on chromosomes 11 and 12 would not 235 have been detected using the traditional marker-regression framework based on final growth (S6 ${ }_{236}$ Fig). 
For a time point calculate AUC

$\mathrm{t}=70$ hours
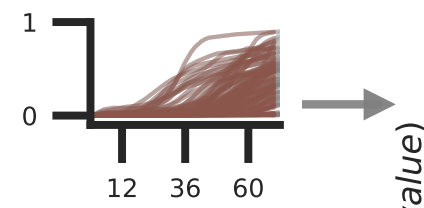

$t=48$ hours

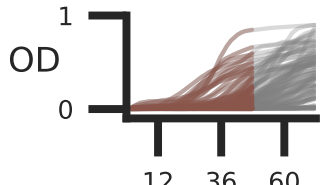

$\mathrm{t}=28$ hours

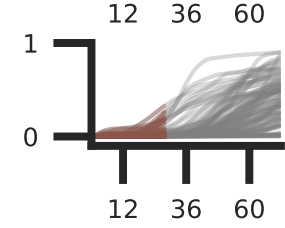

Hours

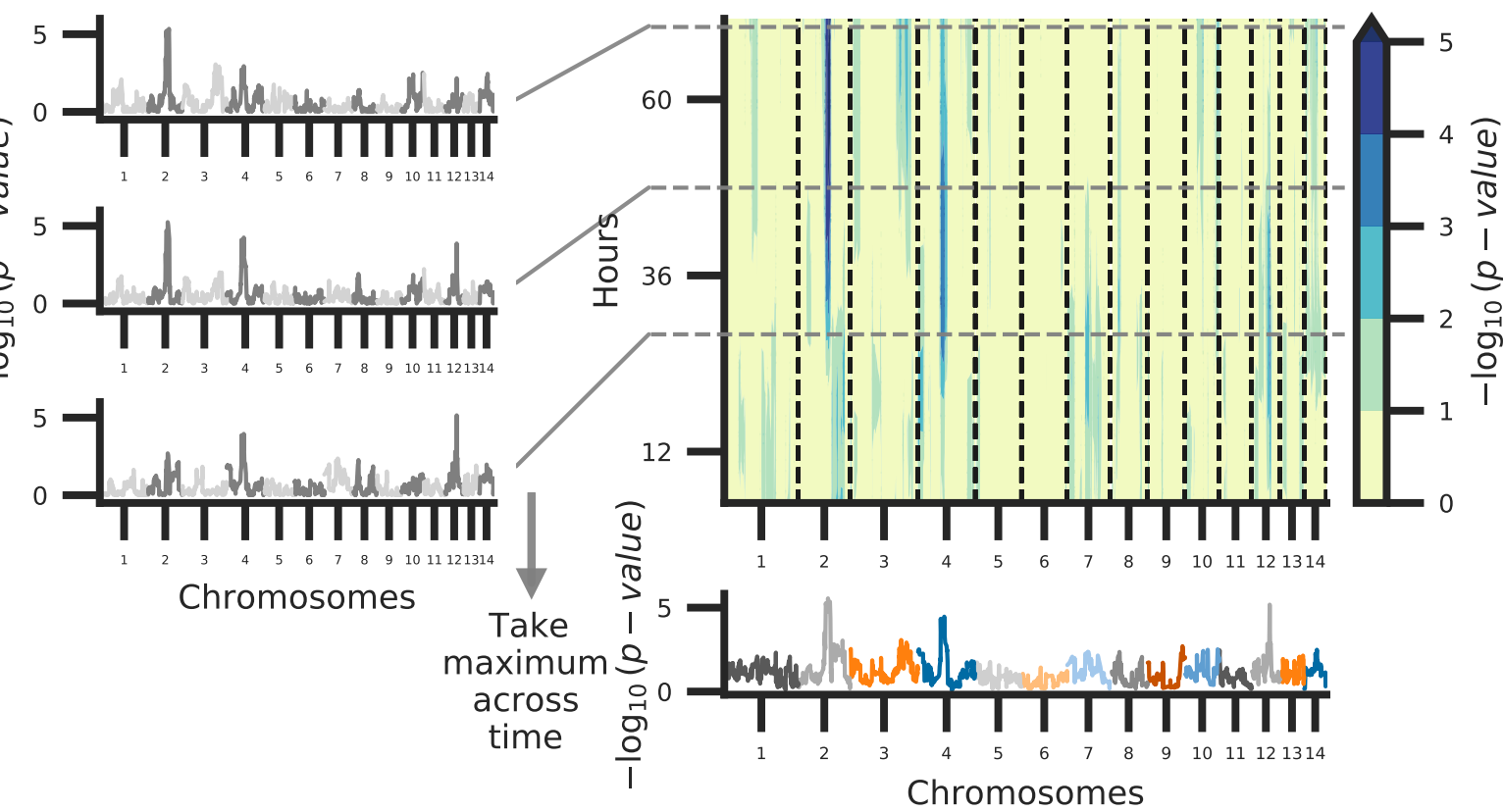

Fig 4. Schematic of temporal QTL mapping. Across experimental conditions, OD was sampled every 15 minutes for 72 hours. Across the 72-hour time course, the median (across replicates) area under the curve (AUC) is calculated per segregant and utilized for QTL mapping, regressing AUC across the 14 chromosomes represented by 3,108 haploblocks. This process is conducted per time point and examples of this analysis at 70,48 , and 28 hours from growth data collected at $37^{\circ} \mathrm{C}$ with $0.125 \mu \mathrm{g} / \mathrm{ml}$ of amphotericin B are depicted. The temporal trends in QTL may then be summarized by taking the maximum per haploblock across time. 
A
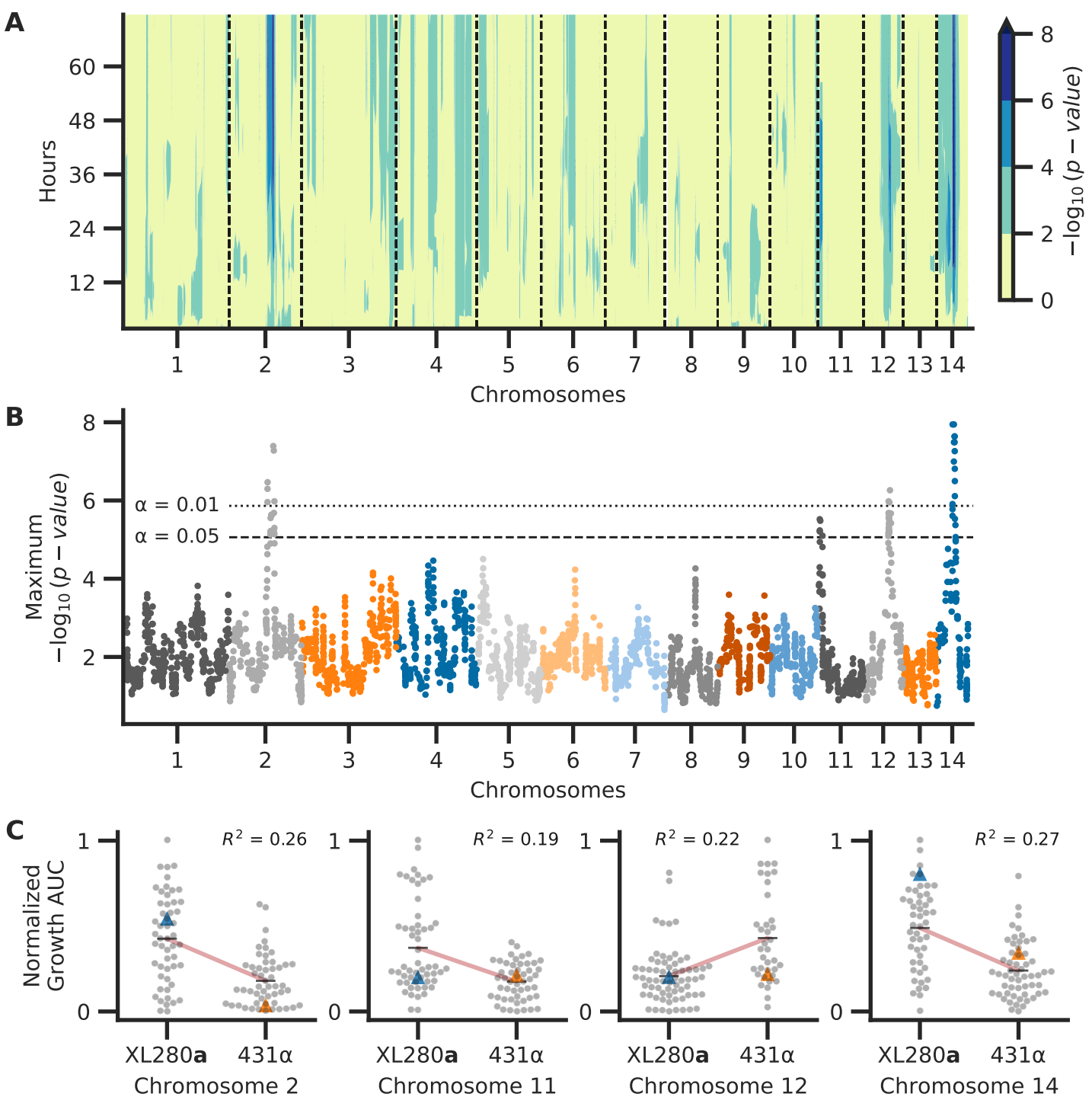

QTL Allele

Fig 5. Associations between genotype and phenotype. Across the experimental combinations of temperature and amphotericin B concentrations in Fig 3, median growth AUCs were regressed onto haplotypes for each sample time point in 72-hour time courses. A) Temporal analysis of association between genotype and phenotype, collapsed across conditions. Across the experimental conditions, the maximum association across time (y-axis) per chromosome (x-axis) is shown. $B$ ) QTL collapsed across conditions and time. The $x$-axis represents chromosomal positions of haploblocks; the $y$-axis represents the maximum - $\log _{10}(p$-value) for each haploblock across both time and conditions. The maximum significance thresholds (dotted and dashed horizontal lines) were determined via permutation. C) Normalized (max-min normalization) AUC of growth (y-axis) by parental allele (x-axis) at the QTL on chromosomes 2, 11, 12, and 14 (left to right, respectively). Blue and orange triangles represent the AUC values for parental strains, black horizontal lines denote phenotypic means by allele, and red lines indicate the best fit line from the regression used to detect QTL. The heritability of each QTL (annotated in black) is estimated by the coefficient of determination from the regression. 


\section{QTL for thermal tolerance}

A QTL on chromosome 14 was identified as having a significant association with growth across 239 the three temperature conditions $\left(30,37\right.$, and $\left.39^{\circ} \mathrm{C}\right)$ with no drug and at $39^{\circ} \mathrm{C}$ with amphotericin $\mathrm{B} \quad 240$ (S4 Fig). The chromosome $14 \mathrm{QTL}$ was strongest at $39^{\circ} \mathrm{C}$ with no amphotericin $\mathrm{B}$ (S5 Fig). This ${ }_{241}$ QTL was thus classified as a high temperature growth QTL. At this locus, segregants possess- 242 ing the XL280a haplotype exhibited greater thermal tolerance and outgrew siblings with the $431 \alpha \quad 243$ haplotype (Fig 5C). This pattern was surprising given that the $431 \alpha$ parental strain is the more 244 thermal tolerant of the parents at $37^{\circ} \mathrm{C}$. The maximum heritability, as estimated by the coefficient 245 of determination from the linear regression QTL model, was approximately 27\%. While this QTL 246 had broad effects across time (Fig 5A, S3 Fig) the maximum association between genotype and 247 phenotype was observed relatively early within the time course at 29 hours.

\section{A pleiotropic QTL governs melanization, capsule size, and thermal tolerance}

QTL for melanization, capsule diameter, and thermal tolerance were mapped to an overlapping is strongly correlated with the capsule size (Spearman $\rho=0.43, p$-value $<0.01$ ) and melaniza- ${ }_{253}$ tion phenotypes (Spearman $\rho=0.51, p$-value $<0.01$ ). However, capsule size and melanization 254 phenotypes displayed only a modest correlation (Spearman $\rho=0.19, p$-value $=0.059$ ). Segre- ${ }_{255}$ gants that displayed greater total growth at $39^{\circ} \mathrm{C}$ had larger capsule diameter or lighter colonies but 256 not necessarily both phenotypes (S7 Fig). Because the capsule diameter and melanization phe- ${ }^{257}$ notypes are strongly correlated with thermal tolerance and because the shared QTL co-localized 258 along chromosome 14, we treated the chromosome 14 locus as a pleiotropic QTL for subsequent 259 analyses.

\section{Multiple QTL for amphotericin B sensitivity}

The QTL on chromosome 2 reached or neared significance in five of the eleven combinations 262 of temperature and amphotericin B concentrations. This QTL was not detected as significant in ${ }_{263}$ any of the conditions lacking amphotericin B, and the maximum association between genotype and 264 
phenotype was observed at $0.125 \mu \mathrm{g} / \mathrm{ml}$ of amphotericin B (S5 Fig). Temporal analysis indicated 265 that during growth in the presence of $0.125 \mu \mathrm{g} / \mathrm{ml}$ amphotericin B, this QTL reached the threshold 266 of significance in the middle of the 72-hour time course (approximately 36 hours) across multiple ${ }_{267}$ temperature conditions and reached its maximum at approximately 65 hours at $30^{\circ} \mathrm{C}$ and 0.125268 $\mu \mathrm{g} / \mathrm{ml}$ amphotericin B (Fig 5A, S3 Fig). This locus was thus designated as an amphotericin B 269 sensitivity QTL. This QTL explains approximately $26 \%$ of the variance in growth at $\sim 65$ hours at 270 $30^{\circ} \mathrm{C}$ and $0.125 \mu \mathrm{g} / \mathrm{ml}$ amphotericin B. Segregants with the $431 \alpha$ haplotype at this QTL were more ${ }_{271}$ susceptible to the fungicidal effects of amphotericin B (Fig 5C). 272

A second amphotericin B QTL was identified on chromosome 11. This QTL was maximally ${ }^{273}$ associated with growth at $37^{\circ} \mathrm{C}$ with $0.175 \mu \mathrm{g} / \mathrm{ml}$ of amphotericin B (S5 Fig). This second QTL ${ }^{274}$ explained $19 \%$ of the phenotypic variation as estimated by the regression model. At this locus, 275 segregants with the XL280a haplotype outgrew their sibling progeny with the $431 \alpha$ haplotype (Fig 276 $5 \mathrm{C})$. The effect of this QTL was seen in the first two-thirds of the 72-hour time course, reaching a 277 maximum at $\sim 40$ hours and trailing off thereafter (S3 Fig).

The QTL identified on chromosome 12 surpassed the significance threshold in three conditions ${ }_{279}$ of high temperature $\left(37^{\circ}\right.$ and $\left.39^{\circ} \mathrm{C}\right)$ and high amphotericin $\mathrm{B}$ concentration $(0.125$ and $0.175 \mu \mathrm{g} / \mathrm{ml}, \quad 280$ S5 Fig). This QTL was designated as a drug associated QTL as it only appeared significant in ${ }_{281}$ conditions with amphotericin B concentrations larger than $0.125 \mu \mathrm{g} / \mathrm{ml}$. At this QTL, segregants 282 with the parental $431 \alpha$ allele outgrew progeny with the XL280a allele. Furthermore, of the QTL ${ }^{283}$ identified here, this was the only QTL that displayed a positive association with alleles from the $431 \alpha \quad 284$ background (Fig 5C). At the highest concentration of amphotericin B, this QTL was maximal near ${ }_{285}$ the middle of the time course ( $\sim 36$ hours) (Fig 5A, S3 Fig). The phenotypic heritability explained ${ }_{286}$ by this locus was estimated to be $\sim 22 \%$.

\section{Identifying candidate genes and nucleotide variants}

For the four QTL detected in temperature and amphotericin B experiments, the regions con- ${ }^{289}$ taining candidate genes were determined by taking the maximum association for each haploblock 290 across time, temperature, and amphotericin B concentration and calculating the left and right 291 boundaries of haploblocks above the maximum significance threshold (across conditions). The 292 
open reading frames of genes within these regions were determined by realigning gene sequences 293 from the JEC21 $\alpha$ reference annotation to the XL280 $\alpha$ reference. For all genes within the four can- 294 didate regions, we predicted potential changes in protein sequence due to the genetic variants 295 between the XL280a and 431 $\alpha$ parental strain (S9 Fig) and identified those with non-synonymous 296 changes (S3 Table). Orthologous genes in the C. neoformans background were identified for ${ }_{297}$ genes with non-synonymous changes between the parental strains. Where available, gene dele- 298 tion strains [21] were used in follow up temperature and amphotericin B growth assays (S10 - 299 S13 Figs). Candidate genes and causal genetic variants were further narrowed down by consult- 300 ing the previous literature, considering the severity of the non-synonymous genetic changes on 301 protein length and function, and comparing the growth curve profiles from temperature and am- 302 photericin B experiments on C. neoformans deletion strains. Using this approach, we identified 303 candidate quantitative trait genes (QTGs) and their associate quantitative trait nucleotides (QTNs) 304 for three of the four QTL identified above (discussed below). For the chromosome 11 QTL we were 305 unable to predict a candidate QTG as several significant non-synonmous changes are observed 306 in genes with unknown function and none of the phenotypes of $C$. neoformans deletion mutants 307 for this region were consistent with our QTL mapping results. Although our analyses focused on 308 coding variants within each QTL region, a summary of non-coding and synonymous variants is 309 included in S3 Table.

\section{RIC8 is a candidate QTG for the pleiotropic QTL on chromosome 14}

The pleiotropic chromosome 14 QTL contributing to variation in melanization, capsule diam- 312 eter, and high temperature growth spanned approximately $69 \mathrm{~kb}$ and was located between the 313 coordinates 354,000 to $423,000 \mathrm{bp}$. There are 29 genes within this QTL region, 17 of which are 314 estimated to have genetic variants that lead to non-synonymous changes between the parental 315 backgrounds (S3 Table). A single-nucleotide polymorphism (SNP) identified in the second to last 316 exon of the gene RIC8 (CNN01270) is predicted to cause a premature stop-gain in the XL280a 317 background when compared to the JEC21 $\alpha$ reference strain (Fig 6). Additional non-synonymous 318 changes in the RIC8 gene were identified in the $431 \alpha$ strain compared to both the XL280a parental 319 strain and the JEC2 $1 \alpha$ reference strain and include an in-frame codon deletion and a predicted 320 
shift in the stop codon (S14 Fig).

Ric8 is a guanine nucleotide exchange factor for $\mathrm{Gpa} 1$, the $\mathrm{G}_{\alpha}$ activator of the cAMP-PKA path- 322 way in Cryptococcus [144]. In C. neoformans, ric8 $\Delta$ strains have been previously demonstrated 323 to exhibit melanization and capsule defects [144]. We confirmed the melanization defect using a 324 ric8 $\Delta$ strain from the $C$. neoformans deletion collection (Fig 7A, S15 Fig). To test the effect of 325 ric8 mutations on thermal tolerance, growth of the ric $8 \Delta$ strain was profiled at $37^{\circ}$ and $39^{\circ} \mathrm{C}$. At ${ }_{326}$ these elevated temperatures, the ric $8 \Delta$ strain exhibited a slower initial growth rate than the wild- ${ }_{327}$ type control strain, but then reached a higher maximum density, with the result being higher total 328 growth (Fig 7B, S15 Fig).

Ric8 loss-of-function mutants are predicted to have lower levels of cAMP signaling [144]. Con- 330 sistent with the finding of Gong et al. [144], the addition of exogenous cAMP to L-DOPA plates 331 restored melanization in the C. neoformans ric8 $\Delta$ strain. The parental strain XL280a, bearing the 332 predicted ric8 loss-of-function allele, also exhibited increased melanization when grown on plates 333 with L-DOPA + cAMP (Fig 7A). The 431 $\alpha$ parent exhibited only modest changes in melanization in 334 the presence of CAMP, suggesting that CAMP-PKA signaling is already active in this background. 335 Because the melanization and thermal tolerance phenotypes of the ric $8 \Delta$ strain were consistent 336 with the effects predicted from the QTL mapping, as were the predicted effects of chemical ma- ${ }_{337}$ nipulation of the XL280a background, the RIC8 allele identified in the XL280a background (RIC8 ${ }_{338}$ XL280a ) was labeled as a likely QTN for melanization, high temperature growth, and capsule size. $\quad 339$

\section{SSK1 is a candidate QTG for amphotericin B sensitivity}

The QTL peak on chromosome 2 spanned approximately 154-kb and was located between 341 coordinates 847,000 and 1,001,000 bp. There are 43 genes within this peak, and 18 of these genes 342 were predicted to have non-synonymous changes between the parental strains (S3 Table). Of ${ }_{343}$ these 18 genes, SSK1 (CNB03090) exhibits the most dramatic difference between the two parental 344 strains. The $431 \alpha$ parental haplotype includes a single base-pair insertion within the second exon 345 that is predicted to cause a frame shift, leading to a premature stop-gain (Fig 6). Because this 346 stop-gain was predicted to truncate more than three-quarters of the Ssk1 protein sequence, the 347 $S S K 1^{431 \alpha}$ variant was categorized as a likely loss-of-function allele. 


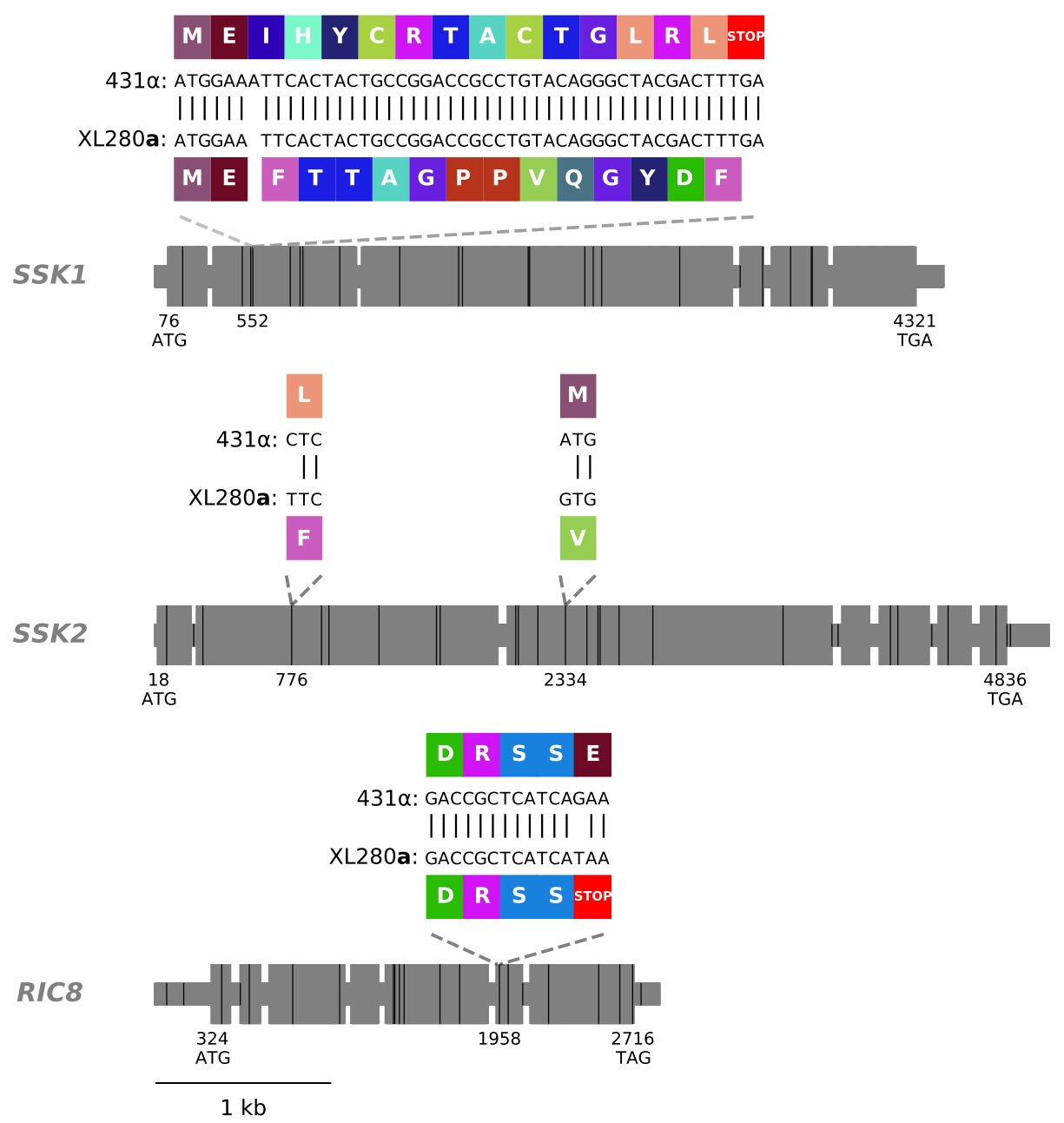

Fig 6. SSK1, SSK2, and RIC8 gene models. Exons are shown as large grey rectangles, while the introns, 5' UTR, and 3' UTR are shown as grey, horizontal lines. The positions of the predicted start and stop codons are annotated along the bottom of the gene bodies and the positions of genetic differences between $431 \alpha$ and XL280a are marked by black, vertical lines. Within the second exon of SSK1, an insertion of a single nucleotide, present in the $431 \alpha$ parental strain is predicted to cause a frame shift that leads to a downstream early stop-gain. Within the second and third exons of SSK2, two SNPs are annotated that lead to non-synonymous changes previously identified by Bahn et al. [143]. Within the second-to-last exon of RIC8, a SNP is present in the XL280a parental strain that is predicted to cause a premature stop. The local, predicted translations of the regions near these non-synonymous, genetic variants and associated amino acids are annotated in colored rectangles. 
A

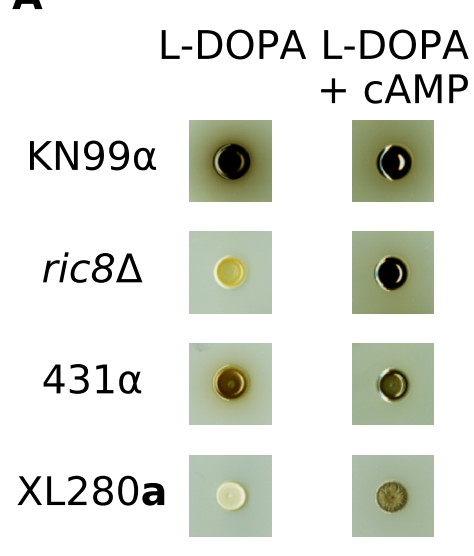

B
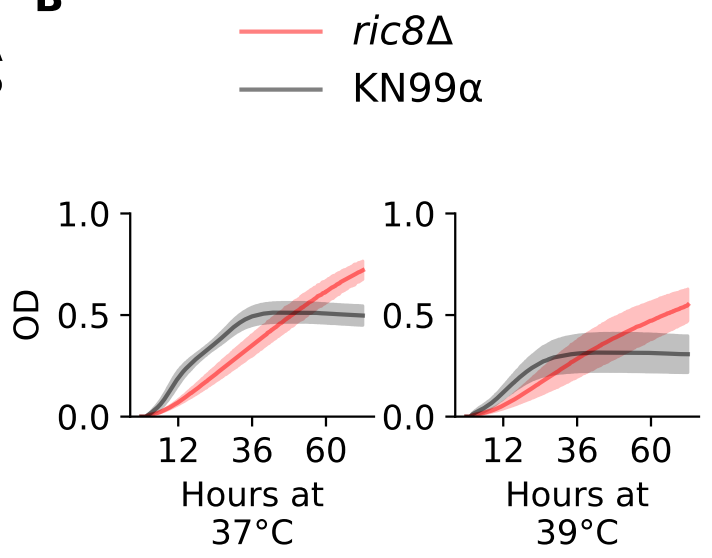

Fig 7. Melanin and high-temperature phenotypes of RIC8. A) The C. neoformans strain, $\mathrm{KN} 99 \alpha$, the ric $8 \Delta$ deletion strain, and $C$. deneoformans parental strains, $431 \alpha$ and XL280a were grown on plates with L-DOPA and L-DOPA + cAMP. Both the ric8 $\Delta$ strain and XL280a demonstrated large increases in the production of melanin when grown in the presence of exogenous cAMP. B) Growth in liquid culture $\left(\mathrm{OD}_{595}, \mathrm{y}\right.$-axis) of KN99 $\alpha$ (black) and the corresponding ric $8 \Delta$ strain (red) under conditions of heat stress $\left(37^{\circ} \mathrm{C}\right.$ and $\left.39^{\circ} \mathrm{C}\right)$ across 72 hours (x-axis).

To provide an independent test of the phenotypic effect of SSK1 loss-of-function mutations, we ${ }_{349}$ phenotyped the ssk1 $\Delta$ strain from the $C$. neoformans gene deletion collection [21]. The ssk1 350 strain in the $\mathrm{H} 99 \alpha$ C. neoformans strain background exhibited an amphotericin B sensitive pheno- 351 type, consistent with the phenotype of segregants bearing the SSK $1^{431 \alpha}$ predicted loss-of-function 352 allele (S16 Fig). Additional ssk1 $\Delta$ strains were constructed in the C. deneoformans, XL280a and 353 $431 \alpha$ parental backgrounds (S1 Table) and phenotyped for amphotericin B sensitivity (S16 Fig). In 354 the $431 \alpha$ strain background, the ssk1 $\Delta$ knockout strain exhibited an amphotericin B sensitive phe- 355 notype, as expected. However, relative to the wild type XL280a strain, none of the XL280a ssk1 356 strains exhibited an amphotericin B sensitive phenotype. We hypothesized this may be due to 357 additional undiscovered allelic variants in this background that also contribute to amphotericin B 358 resistance. 
A fine-mapping procedure was conducted to narrow down the QTL peak on chromosome 2. 361 Specifically, intergenic regions were identified that flank the QTL on chromosome 2 and within 362 these regions, NAT and NEO markers were transformed into the XL280a and $431 \alpha$ parental strains 363 (respectively). From this procedure, one and three transformants were generated in the XL280a 364 and $431 \alpha$ parental strain backgrounds, respectively (S1 Table). Three a- $\alpha$ bisexual crosses were 365 conducted using these marked parental strains, and a large pool of segregants was generated 366 using a random sporulation protocol. From this pool of segregants $173 N A T^{R} N E O^{R}$ segregants, 367 with recombination events within the QTL on chromosome 2 between the two flanking markers, 368 were selected.

Examining the allele frequencies of these progeny, a bias in the SSK1 allele was observed - 370 only $10 \%$ of the population possessed the SSK 1 allele from the $431 \alpha$ parental strain. This was dis- ${ }^{371}$ appointing given the limits on statistical power needed for additional QTL mapping. In this species, 372 centromeres are flanked by crossover hot- and cold-spots [141]. We hypothesized that the proxim- 373 ity of the SSK1 locus to the centromere on chromosome 2 led to a repression of recombination near 374 the left flanking NAT marker, leading to the deviation from the expected 50:50\% allele frequencies 375 (S17 Fig).

\section{SSK2 is also a candidate QTG for amphotericin B sensitivity}

The chromosome 12 QTL spans $\sim 62 \mathrm{~kb}$ and is centered between coordinates 554,000 and 378 $616,000 \mathrm{bp}$. There are 25 genes within this region, 15 of which are predicted to contain non- 379 synonymous changes between the parental strains. Two genes within this region contain a stop- 380 loss and stop-gain, but are hypothetical and of unknown function (S3 Table). Furthermore, deletion 381 strains of these unknown genes in the $\mathrm{H} 99 \alpha$ strain background did not display an amphotericin B 382 sensitive phenotype (S12 Fig).

Among the other candidate genes within this QTL is SSK2 (CNL05560), a MAP kinase of the 384 HOG pathway [143]. By comparing the SSK2 genotypes of the XL280a and $431 \alpha$ parent strains, $\quad 385$ three SNPs were identified that are predicted to cause non-synonymous amino acid differences 386 between the two parental backgrounds (S14 Fig). Two of these non-synonymous SNPs and their ${ }_{387}$ 
associated amino acid changes were previously identified by Bahn et al.[143] and shown to underlie 388

differences in high temperature growth, fludioxonil sensitivity, and osmotic stress responses of 389 C. deneoformans strains (Fig 6).

\section{QTL mapping of HOG-related phenotypes}

Our initial studies of amphotericin B susceptibility implicated two key genes - SSK1 and SSK2 392 - in the HOG pathway, a signaling network that plays a central role in the regulation of cellular 393 responses to osmostress in fungi. Consequently, we predicted that segregants in this study might 394 show variation for additional HOG pathway related phenotypes attributable to one or both of these 395 loci. Thus, we undertook additional analyses of HOG-related phenotypes including resistance to 396 salt stress, resistance to the antifungal drug fludioxonil, and oxidative stress tolerance

\section{QTL for osmotic stress response}

The segregants from this cross were assayed for variation of growth in response to osmotic 399 stress. High resolution images of colonies grown with $1 \mathrm{M} \mathrm{NaCl}$ were made using transmissive 400 imaging (S18 Fig) and the mean grayscale intensity of each colony was used as a proxy for pop- ${ }_{401}$ ulation density. The two parental strains did not vary greatly in their response to salt stress but 402 there was significant growth variation among the segregants (S18 Fig).

A salt tolerance QTL, explaining $\sim 18 \%$ of the phenotypic variance, was identified on chromo- ${ }^{404}$ some 10 (S18 Fig). Segregants with the XL280a allele at the peak of this QTL outgrew sibling ${ }_{405}$ segregants with the $431 \alpha$ allele (S18 Fig). While there are 17 genes within this QTL, none were ${ }_{406}$ identifiable as obvious candidate genes for follow up experimentation (S3 Table).

Fludioxonil is an agricultural antifungal drug whose mode of action is thought to be hyper- ${ }_{409}$ activation of the HOG pathway, leading to physiological effects such as glycerol accumulation 410 and increased turgor pressure [145]. Resistance to fludioxonil has been shown to occur primarily 411 through mutations that ameliorate or decrease HOG signaling. While resistance to fludioxonil is ${ }_{412}$ rare in most fungal species due to the negative pleiotropic consequences of HOG pathway loss- ${ }_{413}$ 
of-function mutations [145], Cryptococcus is unusual in that many strains of both C. deneoformans ${ }_{414}$ and C. neoformans exhibit resistance to this drug. Bahn et al.[143] demonstrated that variation in 415 sensitivity to fludioxonil among Cryptococcus lineages correlates with Hog1 phosphorylation levels ${ }_{416}$ which are in turn correlated with two different allelic states observed at SSK2. The allelic states ${ }_{417}$ identified by Bahn et al. are the same SSK2 alleles identified as segregating in the cross consid- ${ }_{418}$ ered here, with the SSK2 ${ }^{X 280 \mathrm{a}}$ allele predicted to correlate with resistance to fludioxonil and the ${ }_{419}$ SSK2 ${ }^{431 \alpha}$ variant predicted to be sensitive.

Surprisingly, when exposed to fludioxonil $(100 \mu \mathrm{g} / \mathrm{ml})$ the two parental strains both exhibited 421 resistance. We reasoned that the resistance seen in the $431 \alpha$ parental strain was due to an epistatic 422 interaction involving the SSK1 loss-of-function allele identified in this background. Following this ${ }_{423}$ logic, we predicted that recombinant segregants with the SSK1 ${ }^{X L 280 a} S S K 2{ }^{431}{ }^{2}$ genotype would ${ }_{424}$ exhibit sensitivity to fludioxonil. Consistent with this prediction, 14 of the 20 segregants with the ${ }_{425}$ SSK1 ${ }^{\times 280 a}$ SSK2 ${ }^{431 \alpha}$ genotype were fludioxonil sensitive. Segregants with any of the other of ${ }_{426}$ three possible allele combinations at these two loci were fludioxonil resistant (Fig 8).

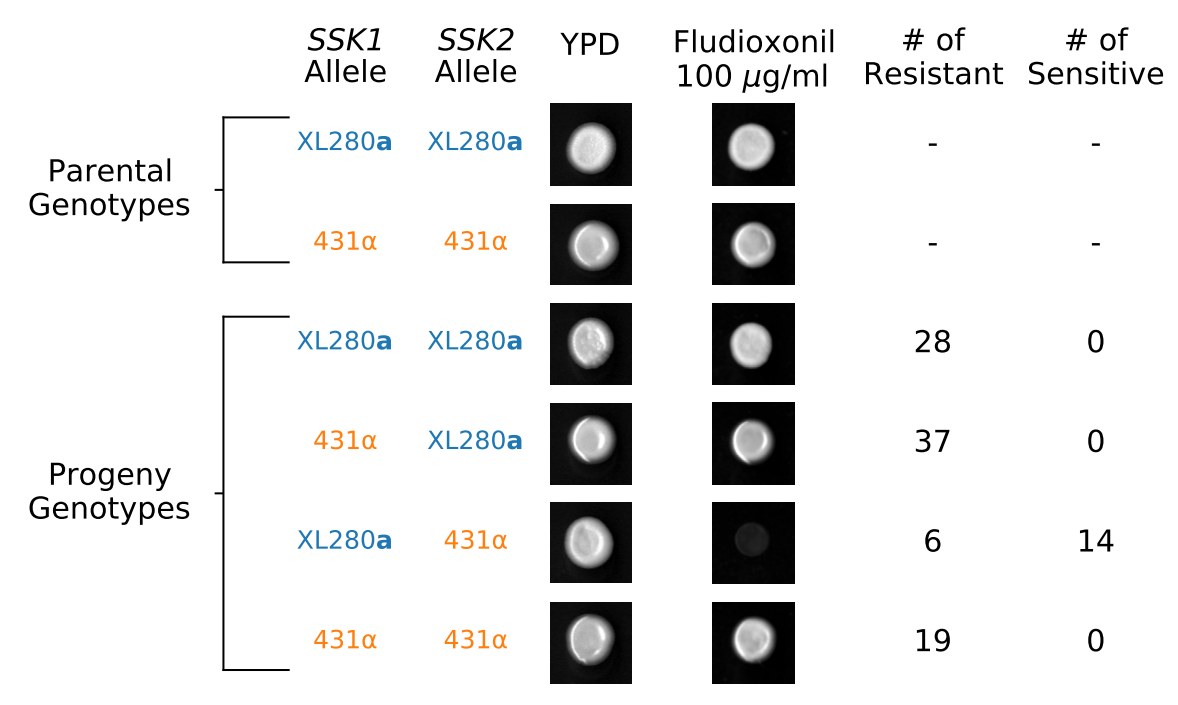

Fig 8. SSK1 and SSK2 govern fludioxonil sensitivity. The relationship between SSK1 and SSK2 genotypes and fludioxonil sensitivity. Only progeny with the SSK $1^{X L 280 a} S S K 2^{431 \alpha}$ genotype are sensitive to fludioxonil. Resistant SSK1 1 L280a $S S K 2^{431 \alpha}$ segregants suggest additional higherorder epistatic interaction involving SSK1 and SSK2.

To provide further evidence for an epistatic interaction between SSK1 and SSK2 we assayed ${ }_{428}$ the additional set of fine-mapped offspring from a- $\alpha$ bisexual crosses between the XL280a strain ${ }_{429}^{42}$ 
and the three $431 \alpha$ strains (S1 Table) for fludioxonil resistance. In this larger set of progeny, only ${ }_{430}$ those segregants that were the genetic mosaics of the XL280a and $431 \alpha$ strains, possessing the ${ }_{431}$ $S S K 1^{\text {XL280a }}$ SSK2 ${ }^{431 \alpha}$ genotype, displayed sensitivity to fludioxonil (S19 Fig). These data sup- ${ }_{432}$ ported our hypothesis that the $S S K 1^{431} \alpha$ allele observed in the $431 \alpha$ parental strain is indeed a ${ }_{433}$ naturally occurring loss-of-function mutation and in this isolate the $S S K 1^{431}{ }^{\alpha}$ has an epistatic effect ${ }_{434}$ with SSK2 ${ }^{431 \alpha}$, rescuing an otherwise fludioxonil-sensitive SSK2 phenotype. Our findings also ${ }_{435}$ point to even higher order genetic interactions - a small number of segregants among those with ${ }_{436}$ the mosaic SSK $1^{X L 280 a}$ SSK2 ${ }^{431} \alpha$ genotype were resistant, indicating the presence of additional ${ }_{437}$ loci that interact epistatically with SSK1 and SSK2 to mediate fludioxonil resistance.

Of the multiple traits examined in this study, fludioxonil sensitivity was the only phenotype to 439 exhibit distinct distributions between segregants derived from $\alpha-\alpha$ unisexual versus a- $\alpha$ bisexual ${ }_{440}$ matings. In the primary mapping population, the majority of fludioxonil sensitive progeny were de- ${ }_{441}$ rived from the unisexual cross. This is due to an allelic bias at the SSK2 locus, wherein bisexually ${ }_{442}$ derived segregants preferentially inherit the SSK2 ${ }^{X L 280 a}$ allele. However, in the fine-mapped off- ${ }_{443}$ spring, all of which are derived from a- $\alpha$ bisexual matings, there is a bias in favor of the SSK2 ${ }_{444}$ $431 \alpha$ allele. Despite these opposing allelic biases, for both unisexually and bisexually derived off- ${ }_{445}$ spring, the only segregants exhibiting fludioxonil sensitivity are those with the allelic combination ${ }_{446}$ SSK1 XL280a $S S K 2^{431 \alpha .}$

\section{SSK2 and RIC8 are QTGs underlying oxidative stress tolerance}

Resistance to oxidative stress is another virulence related trait in Cryptococcus, that is associ- ${ }_{449}$ ated with HOG signaling [143]. Segregants were grown on media containing $5 \mathrm{mM}$ of $\mathrm{H}_{2} \mathrm{O}_{2}$, and 450 colony growth was quantified from high-resolution images by two independent observers using or- ${ }_{451}$ dinal scoring (Fig 9A). There was significant variation in response to $\mathrm{H}_{2} \mathrm{O}_{2}$ across the segregants. ${ }_{452}$ The XL280a parental strain displayed higher tolerance of $\mathrm{H}_{2} \mathrm{O}_{2}$ (on average) than the $431 \propto$ parental ${ }_{453}$ strain, and a portion of the progeny (less than $25 \%$ ) displayed no growth and complete sensitivity ${ }_{454}$ to $\mathrm{H}_{2} \mathrm{O}_{2}$ (Fig 9B). 
A
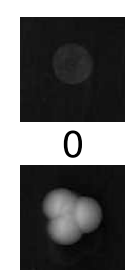

3

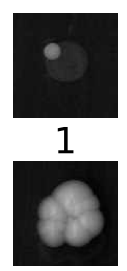

4

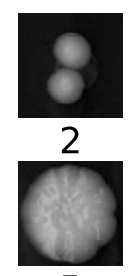

5

$\mathrm{H}_{2} \mathrm{O}_{2}$ Phenotypes \& Scores

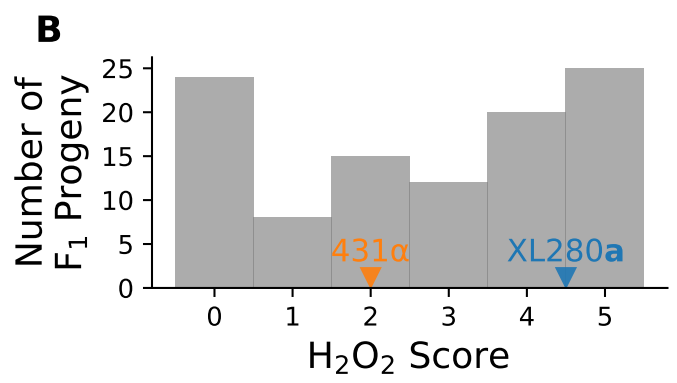

C
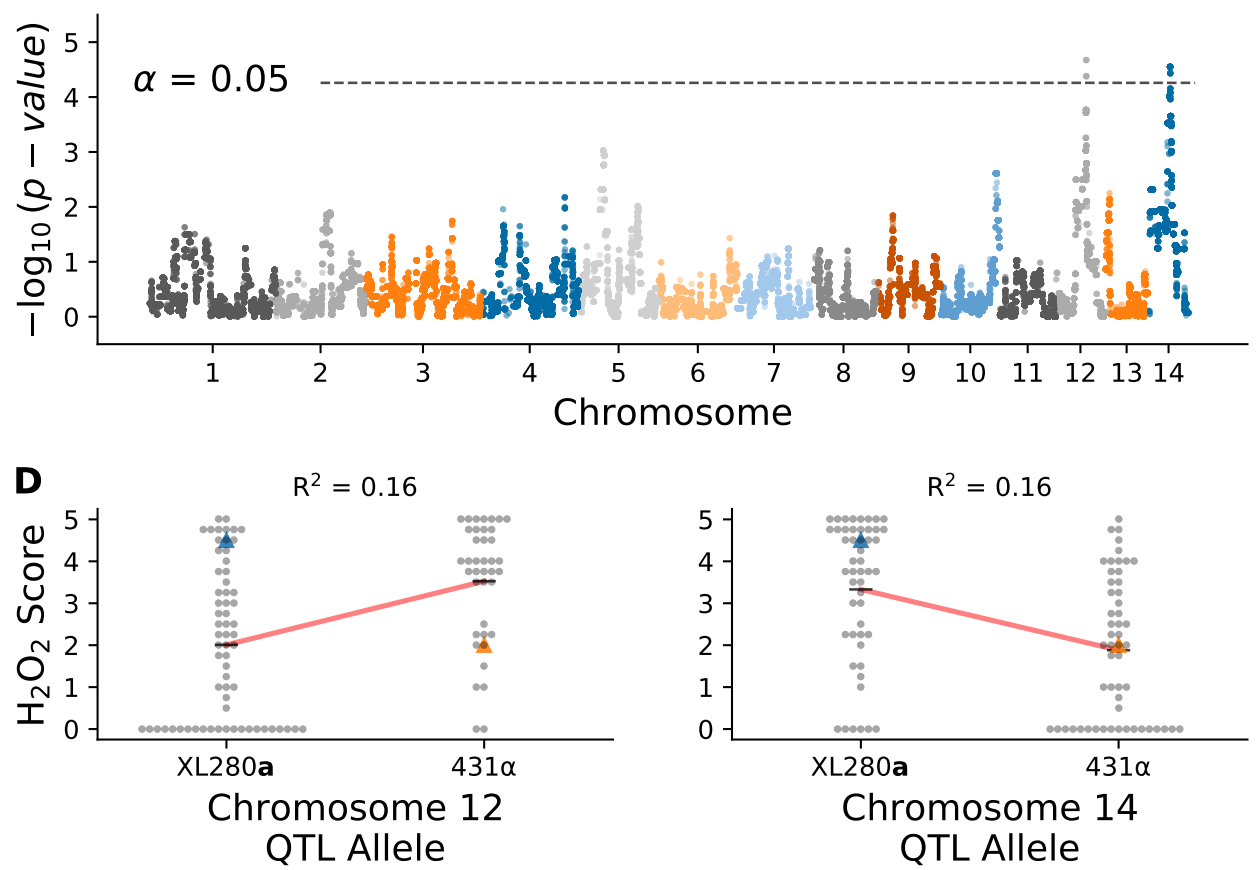

Fig 9. Variation in hydrogen peroxide sensitivity. QTL analysis of variation in response to hydrogen peroxide. A) Example growth phenotypes and scores of $C$. deneoformans segregants grown on media with hydrogen peroxide. B) Histogram of hydrogen peroxide growth scores. Growth scores of parental strains are marked by vertical lines. C) Manhattan plot of the association between genotype and growth in response to hydrogen peroxide. The x-axis represents chromosomal locations of haploblocks and the $y$-axis represents the strength in association between genotype and variation in growth score. D) Hydrogen peroxide growth scores ( $y$-axis) as a function of allele at the peak of chromosome 12 (left) and chromosome 14 (right) QTL. Blue and orange triangles mark the parental phenotypes, black horizontal lines denote the average phenotypes of segregants by allele, and red lines are regression models relating the genotype to phenotype. The heritablity at each locus is estimated from these models and annotated in black. 
Two QTL associated with hydrogen peroxide growth were detected (Fig 9C), on chromosomes 456 12 and chromosome 14, each with modest heritablity ( 16\% at each QTL); (Fig 9D). These two ${ }_{457}^{45}$ QTL overlapped with the previously identified QTL governing amphotericin B sensitivity on chro- ${ }_{458}$ mosome 12 and the pleiotropic QTL underlying variation in melanization, capsule size, and thermal ${ }_{459}$ tolerance on chromosome 14. Joint analysis of the $\mathrm{H}_{2} \mathrm{O}_{2}$ growth scores and growth at $37^{\circ} \mathrm{C}$ with ${ }_{460}$ $0.125 \mu \mathrm{g} / \mathrm{ml}$ of amphotericin B revealed a strong correlation between these phenotypes (Spear- ${ }_{461}$ man $\rho=0.5, p$-value $<7^{-8}$ ). Segregants with the $431 \alpha$ allele at the chromosome 12 QTL peak ${ }_{462}$ display greater tolerance to $\mathrm{H}_{2} \mathrm{O}_{2}$ and resistance to amphotericin B (S20 Fig). Similarly, for the ${ }_{463}$ virulence-related phenotypes associated with the chromosome $14 \mathrm{QTL}$, the $\mathrm{H}_{2} \mathrm{O}_{2}$ phenotype was ${ }_{464}$ strongly correlated with thermal tolerance at $39^{\circ} \mathrm{C}$ (Spearman $\rho=0.53, p$-value $<9^{-9}$ ) and nega- ${ }_{465}$ tively correlated with melanization (Spearman $\rho=-0.59, p$-value $<5^{-11}$ ). There was no significant 466 correlation between $\mathrm{H}_{2} \mathrm{O}_{2}$ growth and the capsule phenotype (Spearman $\rho=0.15, p$-value $=0.13$ ). ${ }_{467}$ Given the location of $\mathrm{H}_{2} \mathrm{O}_{2}$ QTLs, and the general trends of phenotypic correlations observed, we ${ }_{468}$ predict that SSK2 (chromosome 12) and RIC8 (chromosome 14) are the underlying QTGs for this ${ }_{469}$ trait.

At the predicted QTN for SSK2 and RIC8 the marginal variance explained was approximately ${ }_{471}$ $13 \%$ and $14 \%$ (respectively). A linear model based solely on additive effects of these two loci 472 explains approximately $21 \%$ of phenotypic variance, while models that include an interaction term 473 between these loci explains $26.4 \%$ of the variance (ANOVA, $p$-value $<1.0^{-6}$ ). Segregants with 474 the SSK2 ${ }^{\mathrm{XL280a}} \mathrm{RIC} 8^{431 \alpha}$ genotype displayed the greatest average sensitivity to $\mathrm{H}_{2} \mathrm{O}_{2}$ while other ${ }_{475}$ allelic combinations of SSK2 and RIC8 exhibited similar average $\mathrm{H}_{2} \mathrm{O}_{2}$ resistance (S21 Fig). 476

Re-examination of thermal tolerance phenotypes (Fig 3) with respect to two-locus SSK2 RIC8 477 genotypes, suggests that epistasis between these two loci may also influence this trait. Across 478 the temperatures used in growth curve assays $\left(30,37,39^{\circ} \mathrm{C}\right)$, segregants bearing the SSK2 479 XL280a $R / C 8^{431 \alpha}$ genotype displayed the poorest overall growth (S21 Fig). This growth defect was ${ }_{480}$ most pronounced at $39^{\circ} \mathrm{C}\left(\right.$ ANOVA, $R^{2}=0.332, p$-value $\left.<9^{-9}\right)$. 
Since SSK2 was found to interact epistatically with both SSK1 (fludioxonil resistance) and RIC8 483 (oxidative stress resistance and thermal tolerance), we hypothesized that higher-order interactions ${ }_{484}$ involving all three loci might contribute to phenotypic variation in one or more of these traits. To 485 test for three-way epistasis, we employed an approach proposed by Hu et al.[146], which uses a 486 statistic called "information gain" (IG), which is based on information-theoretic mutual information 487 measures [147]. Hu et al.'s IG statistic provides a measure of synergistic interaction between three ${ }_{488}$ loci with respect to a phenotype of interest, after subtracting the information inherent in single locus ${ }_{489}$ effects and synergies between pairs of loci. Since the IG statistic requires discrete data, we lim- 490 ited our analysis of three-way epistasis to a transformed $\mathrm{H}_{2} \mathrm{O}_{2}$ resistance phenotype, classifying 491 each segregant as either sensitive, intermediate, or resistant. Applying the IG method to $\mathrm{H}_{2} \mathrm{O}_{2}{ }_{492}$ resistance, we find evidence for single locus effects $\left(I G(S S K 1)=4.6 \%\right.$, p-value $=0.005$; IG(SSK2) ${ }_{493}$ $=6.0 \%, p$-value $=0.002 ; \operatorname{IG}(R / C 8)=5.4 \%, p$-value $=0.006)$ as well as a three-way synergy be- ${ }_{494}$ tween SSK1, SSK2, and RIC8 (IG(SSK1,SSK2,RIC8) = 7.0\%, p-value = 0.004), but no significant ${ }_{495}$ pairwise synergies.

Fig $10 \mathrm{~A}$ illustrates the distributions of $\mathrm{H}_{2} \mathrm{O}_{2}$ growth scores for each of the eight possible geno- ${ }_{497}$ typic combinations of SSK1, SSK2, and RIC8. The mapping between the three-locus genotypes 498 and $\mathrm{H}_{2} \mathrm{O}_{2}$ resistance can be summarized as follows. Segregants with the two-locus genotypic 499 combination SSK2 ${ }^{X 280 a} R / C 8^{431 \alpha}$ exhibit the lowest average $\mathrm{H}_{2} \mathrm{O}_{2}$ resistance. Conversely, seg- 500 regants with the opposite genotypic combination $S S K 2^{431 \alpha} R / C 8^{X L 280 a}$ exhibit high average $\mathrm{H}_{2} \mathrm{O}_{2} \quad{ }_{501}$ resistance. The phenotype of segregants with the other two-locus combinations of SSK2 and RIC8 502 (i.e. SSK2 ${ }^{\mathrm{XL280a}} R / C 8^{\mathrm{XL280a}}$ and $S S K 2^{431 \alpha} R I C 8^{431 \alpha}$ ) depends on their genotypic state at SSK1- ${ }_{503}$ those with the SSK1 ${ }^{X 280 a}$ allele have high average resistance, while those with the SSK $1^{431 \alpha}$ allele ${ }_{504}$ exhibit intermediate average resistance. 
A

SSK1, SSK2, and RIC8 genotypes

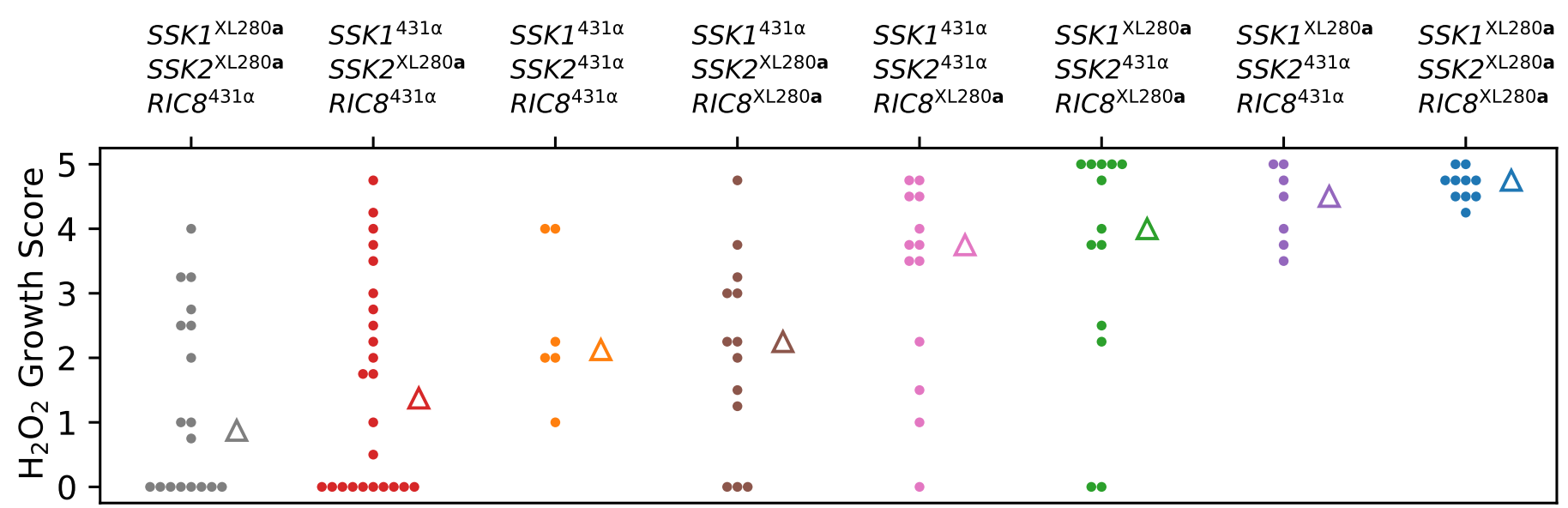

B

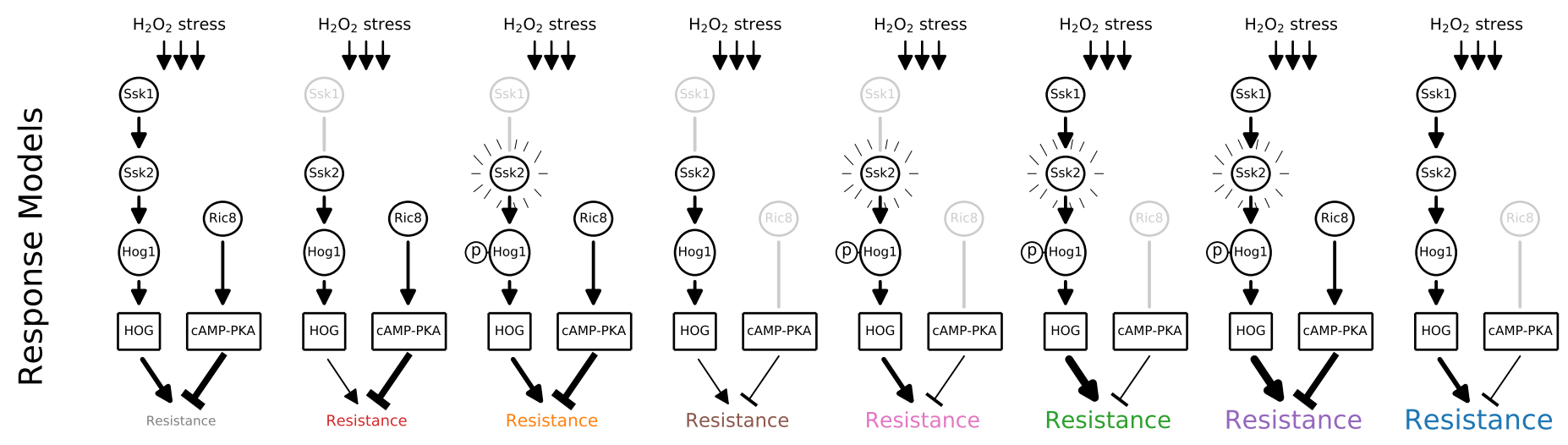

Fig 10. Three-way epistasis underlies $\mathrm{H}_{2} \mathrm{O}_{2}$ resistance. Three-way epistasis between $S S K 1$, SSK2, and RIC8 governs resistance to $\mathrm{H}_{2} \mathrm{O}_{2}$ stress. A) Distributions of growth scores under $\mathrm{H}_{2} \mathrm{O}_{2}$ stress (y-axis) for segregants with different three locus genotypes at $\mathrm{SSK} 1$, SSK2, and RIC8 (x-axis and color). Triangles mark the median scores per genotype. B) Proposed models for HOG and cAMP-PKA signaling for each genotypic combination in $\mathbf{A}$. The $S S K 1^{431 \alpha}$ and $R I C 8^{X L 280 a}$ alleles are predicted to be non-functional and associated with reduced signaling. The SSK2 ${ }^{431} \alpha$ allele has been previously associated with increased basal levels of Hog 1 phosphorylation. Proposed levels of HOG and cAMP-PKA signaling are denoted by edge thickness of expression and repression arrows. 


\section{The $R I C 8^{\mathrm{XL280a}}$ allele likely arose during laboratory passage}

Allelic variation at the RIC8 gene, which is predicted to affect cAMP-PKA signaling, was asso- ${ }_{507}$ ciated with phenotypic variation for a large number of virulence traits. Given its prevalence across 508 our mapping experiments, we sought to identify the ancestral source of the premature stop-gain 509 allele observed in the XL280 backgrounds. The strains XL280a and XL280 $\alpha$ SS are laboratory 510 generated strains [137], and analysis of ancestral, progenitor strains used in their construction 511 revealed that the RIC8 stop-gain allele is also found within the strain B3502 [148]. However, this 512 allele was not present in either progenitor strains used to construct B3502. We therefore concluded 513 that the premature stop-gain in the $R / C 8^{\mathrm{XL280}}$ allele is inherited from the strain $\mathrm{B} 3502$ and is a de 514 novo mutation generated during its construction. The findings of prior studies that have used the 515 B3502 background [e.g. 52, 70, 143, 149-151] should be evaluated in light of potential abrogation 516 of cAMP-PKA signaling.

\section{Discussion}

The work presented here is the highest resolution QTL mapping study to date in a human ${ }_{519}$ fungal pathogen $[79,110,111]$. We mapped QTL for multiple virulence-associated traits as well 520 as resistance to two widely used antifungal drugs. By exploiting the detailed SNP information that ${ }_{521}$ whole-genome sequence data provides, we subsequently identified specific genes (Quantitative 522 Trait Gene; QTG) and nucleotide variants (Quantitative Trait Nucleotide; QTN) that are likely to 523 underlie those QTL. Of particular note is the fact that the three QTG we identified are regulators of ${ }_{524}$ signaling pathways - the cAMP-PKA pathway and the HOG pathway - that are important for fungal ${ }_{525}$ adaptation to extracellular stresses $[152,153]$ and have been shown to be integral to virulence in 526 Cryptococcus $[24,154]$. Both of these pathways regulate multiple physiological and morphological $\quad 527$ traits in Cryptococcus as well as other fungi including S. cerevisiae [155], Candida albicans [26, 528 156], and Candida auris [157]. 


\section{Allelic variation in CAMP-PKA signaling has highly pleiotropic effects on virulence 530 traits

QTL for melanization, thermal tolerance, capsule size, and growth under oxidative stress $\left(\mathrm{H}_{2} \mathrm{O}_{2}\right) \quad 532$ all mapped to the same approximate region on chromosome 14. We identified a likely QTN for this 533 pleiotropic QTL - a SNP that leads to a premature stop-gain within the penultimate exon of the gene ${ }_{534}$ RIC8. This genetic variant is present in the XL280a parental strain. Ric8 is a guanine nucleotide 535 exchange factor for the $\mathrm{G}_{\alpha}$ protein Gpa1, which activates the cAMP-PKA signaling pathway in 536 C. neoformans $[144,158]$. Based on the location of this variant, we predict that the XL280a allele 537 results in a loss-of-function, perhaps partial, of $R / C 8$, and hence reduces signalling through the 538 cAMP-PKA pathway.

In our mapping population the $R / C 8^{X L 280 a}$ allele was associated with decreased melanization 540 but increased thermal tolerance, capsule size, and $\mathrm{H}_{2} \mathrm{O}_{2}$ resistance. The associations we ob- ${ }_{541}$ served between phenotypes and R/C8 genotypes in our $C$. deneoformans mapping population 542 show a mixture of agreement and disagreement with prior studies of cAMP-PKA signaling in Cryp- ${ }_{543}$ tococcus, most of which have been conducted in C. neoformans. For example, Gong et al. [144] 544 showed that in C. neoformans, ric8 $\Delta$ mutants exhibit a loss of melanization and reduced capsule ${ }_{545}$ size, and that both of these phenotypes could be rescued by cAMP supplementation. We found 546 that addition of exogenous cAMP to the growth medium increased melanization of the XL280a ${ }_{547}$ strain, consistent with the prediction that the $R / C 8^{\mathrm{XL280a}}$ is associated with reduced CAMP sig- 548 naling. However, contrary to that prior study, the predicted loss-of-function R/C8XL280a allele was ${ }_{549}$ associated with increased relative capsule size.

Gong et al. [144] did not examine thermal tolerance in their study, but we assayed growth of a 551 C. neoformans ric8 $\Delta$ strain at high temperatures, and found that this strain exhibited slower initial 552 growth rates relative to the wild-type background, but came to a higher overall population density. 553 This parallels the thermal tolerance phenotype we observed for the XL280a background. A similar ${ }_{554}$ inverse relationship between thermal tolerance and cAMP-PKA signaling has been observed in S. 555 cerevisiae; Li et al. [159] found that hyperactivation of CAMP-PKA signaling reduces resistance to 556 acute heat stress while PKA inhibition increased resistance. 
is negatively correlated with thermal tolerance and $\mathrm{H}_{2} \mathrm{O}_{2}$ resistance; b) capsule size is positively 559 correlated with thermal tolerance but weakly correlated with melanization and $\mathrm{H}_{2} \mathrm{O}_{2}$ resistance; and 560 c) $\mathrm{H}_{2} \mathrm{O}_{2}$ resistance is positively correlated with thermal tolerance. While melanization is thought 561 to be protective against reactive oxygen species [160], Jacobson et al. [70] observed no signifi- 562 cant protection against $\mathrm{H}_{2} \mathrm{O}_{2}$ in $\mathrm{C}$. deneoformans strains; thus observing a negative relationship ${ }_{563}$ between these two phenotypes is not unexpected. However, the inverse relationship between 564 melanization and thermal tolerance is somewhat surprising, as prior studies have demonstrated a 565 positive relationship between the production of melanin and the ability to grow at high temperatures 566 [68].

A likely explanation for the mix of similar and dissimilar correlates with RIC8 genotypes rela- ${ }_{568}$ tive to earlier work, is divergence in CAMP-PKA signaling between C. neoformans and C. dene- 569 oformans strains. Hicks et al. and Hicks and Heitman [161, 162] showed that mutations of the 570 PKA catalytic subunits, PKA1 and PKA2, have distinctly different effects on melanization, capsule 571 formation, and mating in C. neoformans, C. deneoformans and the more distantly related species 572 C. gattii. Genetic variation that affects CAMP-PKA signaling in particular is an increasingly common 573 theme in studies of fungal quantitative genetics and experimental evolution [163-166]. A recent 574 comparative study of cAMP-PKA signaling in S. cerevisiae and related yeasts hypothesized that 575 this pathway is likely to be a hotspot for functional variation and evolutionary adaptation [167]. We 576 predict that segregating genetic variation in CAMP-PKA signaling may be particularly relevant for 577 natural variation in virulence-related traits not only in Cryptococcus but in other pathogenic fungi 578 as well.

\section{HOG pathway variants moderate resistance to the antifungal drug amphotericin B}

Major QTL for amphotericin B sensitivity were found on chromosomes 2 and 12 . We identified ${ }_{581}$ candidate QTG that underlie these loci - SSK1 on chromosome 2 and SSK2 on chromosome 12582 - both of which are components of the HOG signalling pathway. The HOG pathway is known to 583 regulate the production of ergosterol, the target of amphotericin B [168]. 584

At the chromosome 2 locus we discovered a single nucleotide insertion in the $431 \alpha$ parental 585 strain background that leads to an early stop-gain in the gene SSK1, the response regulator of the 586 
HOG pathway. Given the location of the identified variant, it is likely that this results in a complete 587 loss of function for the gene. C. neoformans ssk1 $\Delta$ mutant strains exhibit increased sensitivity to 588 amphotericin B compared to wild-type strains [148, 168], a pattern we observed in segregants with 589 the SSK $1^{431 \alpha}$ allele. Interestingly, the ssk1 $\Delta$ strains in the XL280a background we generated did 590 not display an amphotericin B sensitive phenotype. We hypothesize that this was due to additional 591 genetic variants in this background that also contribute to amphotericin B resistance. 592

In C. neoformans, but not C. deneoformans strains, Ssk1 has also been shown to govern ${ }^{593}$ capsule elaboration and melanization [169]. Consistent with this earlier study, allelic variation at 594 SSK1 is not associated with variation in either capsule or melanin phenotypes in our mapping 595 population, suggesting that HOG signaling has diverged between Cryptococcus species. 596

The likely causal variants for amphotericin B sensitivity that mapped to chromosome 12 are al- $\quad 597$ leles of the gene SSK2, the MAPKKK of the HOG pathway. The variant sites within SSK2 observed 598 here have been previously described by Bahn et al. [143]. In their study, Bahn et al. [143] showed 599 that the allele present in the $431 \alpha$ parental strain $\left(S S K 2^{431 \alpha}\right.$ ) is associated with increased basal 600 levels of Hog1 phosphorylation and in a follow-up study, Ko et al. [168] demonstrated that C. de- ${ }_{601}$ neoformans strains with this allele are more resistant to higher concentrations of amphotericin B. 602 Across C. neoformans and C. deneoformans strains, the amount of pre-phosphorylated Hog1 is ${ }_{603}$ known to vary [169], and Bahn et al. [143] hypothesized that this allowed some strains to rapidly 604 respond to extracellular stresses. Interestingly in our study, SSK2 ${ }^{431 \alpha}$ was the only allele from the ${ }_{605}$ $431 \alpha$ parental strain associated with increased fitness.

\section{Epistatic interactions within and between signaling pathways}

Ssk1 and Ssk2 are both members of the HOG pathway, and Ssk1 physically interacts with and ${ }_{608}$ regulates Ssk2's kinase activity [24, 153, 170]. This naturally led us to explore genetic interactions 609 between the allelic states we observed for SSK1 and SSK2. Additionally, crosstalk between HOG 610 and cAMP-PKA signaling pathways has been documented in both S. cerevisiae and C. neoformans 611 [169, 171-174], thus motivating an exploration of genetic interactions between R/C8 and HOG- 612 pathway alleles.

We found that an epistatic interaction between SSK1 and SSK2 affected sensitivity to fludiox- 
onil, an agricultural antifungal drug. Fludioxonil's mode of action is thought to be hyperactivation of 615 the HOG pathway [175] and resistance to fludioxonil occurs primarily through HOG pathway loss- 616 of-function mutations $[145,176]$. Bahn et al. [143] showed that strains with the SSK2 ${ }^{431}{ }^{\alpha}$ genotype ${ }_{617}$ were sensitive to fludioxonil, presumably due to hyperactive HOG signaling associated with pre- ${ }_{618}$ phosphorylated Hog1. Surprisingly, the parental strain $431 \alpha$, which bears the predicted sensitive ${ }_{619}$ SSK2 allele, was fludioxonil resistant. Analysis of offspring from our cross revealed that the unex- ${ }_{620}$ pected resistance in $431 \alpha$ is mediated by allelic variation at SSK1 - segregants with the sensitive ${ }_{621}$ $S S K 2^{431 \alpha}$ allele as well as the loss-of-function SSK1 ${ }^{431 \alpha}$ allele exhibit fludioxonil resistance, while ${ }_{622}$ the pairing of the sensitive SSK2 ${ }^{431} \alpha$ allele with the functional SSK1 $\times$ L280a allele results in sensitiv- ${ }_{623}$ ity to fludioxonil. All other allelic combinations at these two loci are fludioxonil resistant. This thus ${ }_{624}$ represents a compelling example of within-pathway epistasis. 625

Our analysis of hydrogen peroxide resistance suggests complex cross-pathway epistasis be- ${ }_{626}$ tween the HOG and CAMP-PKA signaling networks. We propose that the three-way epistatic in- ${ }_{627}$ teraction between SSK1, SSK2, and RIC8 can be rationalized in terms of the relative balance ${ }_{628}$ between HOG signaling and CAMP-PKA signaling (Fig 10B). Segregants with the genotype SSK2 ${ }_{629}$ $\mathrm{XL280a} R / C 8^{431 \alpha}$ exhibit the greatest average sensitivity to hydrogen peroxide stress. This geno- ${ }_{630}$ typic combination is predicted to have weak or intermediate levels of HOG signaling but normal ${ }_{631}$ CAMP-PKA signaling. Conversely, segregants with the genotype SSK2 ${ }^{431 \alpha}$ RIC $8^{\mathrm{XL} 280 \mathrm{a}}$ are pre- ${ }_{632}$ dicted to have intermediate or high HOG signaling activity (associated with pre-phosphorylation ${ }_{633}$ of Hog1), but weak cAMP-PKA signaling (due to Ric8 loss-of-function alleles), resulting in $\mathrm{H}_{2} \mathrm{O}_{2} \quad 634$ resistance. The two genotype combinations are thus consistent with the observed marginal ef- 635 fects of SSK2 and RIC8. The phenotypes of the other genotypic combinations of SSK2 and RIC8 ${ }^{636}$ require consideration of SSK1 allelic state. For example, intermediate resistance phenotypes are ${ }_{637}$ associated with either intermediate HOG signaling (SSK1 ${ }^{431} \alpha$ SSK2 $\left.{ }^{431} \alpha\right)$ combined with normal ${ }_{638}$ cAMP-signaling $\left(R I C 8^{431 \alpha}\right)$ or weak HOG signaling $\left(S S K 1^{431 \alpha} S S K 2\right.$ XL280a $)$ coupled with weak ${ }_{639}$ CAMP-signaling $\left(R / C 8^{X L 280 a}\right)$.

There is significant experimental evidence consistent with a model of opposing effects of HOG ${ }_{641}$ and cAMP-PKA signaling for a variety of stress responsive phenotypes in both Cryptococcus and ${ }_{642}$ other fungi. For example, Maeng et al. [177] report that $C$. neoformans ras1 $\Delta$ mutants show ${ }_{643}$ increased resistance to $\mathrm{H}_{2} \mathrm{O}_{2}$ stress but decreased resistance to diamide, while hog1 $\Delta$ mutants ${ }_{644}$ 
are $\mathrm{H}_{2} \mathrm{O}_{2}$ sensitive and diamide resistant. C. neoformans hog1 $\Delta$ mutants exhibit an increase ${ }_{645}$ in capsule size [169], another stress responsive phenotype, while cAMP related mutations such 646 as gpa1 $\Delta$, pka1 $\Delta, \operatorname{cac} 1 \Delta$, and ric8 $\Delta$ have reduced or absent capsule [144, 178-180]. Gutin et ${ }_{647}$ al. [173] report evidence of crosstalk between cAMP-PKA and HOG signaling with respect to the ${ }_{648}$ general stress response in yeast, with cAMP-PKA activity associated with the repression of key ${ }_{649}$ stress responsive genes while HOG activity is associated with their activation.

A critical feature of our study is the inclusion of temporal data and the application of function- ${ }^{652}$ valued analytical approaches for key growth traits. While there are both experimental [181] and 653 statistical $[134,135]$ challenges associated with the collection of high-resolution time series growth 654 phenotypes and functional valued QTL mapping, such data are much more information rich than 655 conventional end-point growth or estimates of maximum growth rates. For example, several of the ${ }^{656}$ QTL associations we identified are temporally variable and would likely not have been detected 657 without the inclusion of a time axis in our analyses.

The importance of temporal information for microbial growth phenotypes is not limited to QTL ${ }_{659}$ mapping, but is equally relevant to genetic approaches based on mutational analysis. For ex- 660 ample, we found that when exposed to heat stress $C$. neoformans ric $8 \Delta$ mutants exhibit slower ${ }_{661}$ maximum growth rates compared to an isogenic wild-type strain, but reach a higher final popula- ${ }_{662}$ tion density (Fig 7B). The determination of whether the mutant grows better or worse than wild-type ${ }_{663}$ thus depends critically on when during growth this question is posed and whether the investigator ${ }_{664}$ considers maximal growth rate or final population density to be a better reflection of thermal toler- ${ }_{665}$ ance. Only by including the time axis and using a function valued approach can we appreciate the ${ }_{666}$ complexity of growth patterns inherent in this comparison.

While not explored in this report, we hypothesize that the relative timing of QTL or mutational $\quad{ }_{668}$ associations with microbial growth phenotypes may reflect key transitions in physiological state ${ }_{669}$ within microbial populations. Temporal QTL/mutational information could thus be used to inform the 670 construction of models relating the dynamical behavior of gene networks to distinct physiological ${ }_{671}$ states. 


\section{Comparison to previous QTL studies in Cryptococcus and other fungi}

There are commonalities between the results presented here and a previous QTL mapping ${ }_{674}$ study of C. deneoformans. For example, Lin et al. [111] examined variation in thermal tolerance 675 and melanization within C. deneoformans progeny and identified a pleiotropic QTL on chromosome 676 7 that contributes towards both of these aforementioned traits. This QTL was narrowed down to 677 allelic differences in MAC1, a copper homeostasis transcription factor. Similar to this past study, 678 we also identified pleiotropic QTGs contributing to more than one virulence-related trait examined 679 here (including thermal tolerance and melanization), namely the genes RIC8 and SSK2. While our ${ }_{680}$ study did not share any of the previously implicated QTGs [79, 111], across several studies - not ${ }_{681}$ just those using QTL mapping strategies - observing pleiotropic effects of genes and pathways ${ }_{682}$ connected to virulence and virulence-associated traits seems to be a unifying phenomena [108, 683 182-187].

\section{The genetic complexity of virulence phenotypes}

While the effects of each of the QTL we identified are relatively large, explaining on average ${ }_{686}$ $24 \%$ of the phenotypic variance in amphotericin B susceptibility at SSK1 and SSK2, $33 \%$ in ${ }_{687}$ thermal tolerance between SSK2 and $R / C 8,17 \%$ in capsule size, and $39 \%$ in melanization at $R / C 8, \quad{ }_{688}$ and $25 \%$ of variation in resistance to $\mathrm{H}_{2} \mathrm{O}_{2}$ between SSK2 and $R / C 8$, there are still large portions ${ }_{689}$ of unexplained phenotypic variation. Coupled with the observation of transgressive phenotypes 690 in several of our experiments, these data may suggest the presence of many unidentified QTL ${ }_{691}$ and undiscovered epistatic interactions. Our analysis also focused primarily on the analysis of 692 non-synonymous coding variants, though non-coding variation (e.g. [188, 189]) and synonymous ${ }_{693}$ variation (e.g. [190]) have both been shown to be important for the genetic architecture of complex 694 traits in fungi and as well as other eukaryotes. Additional functional analyses, larger mapping 695 populations and higher order models that test for multiple [191] and interacting loci [192] may help 696 to detect these elusive QTL in future studies. 


\section{Implications for the study of fungal virulence}

Virulence is a complex outcome, an emergent property, that is determined by the combined effects of numerous morphological, physiological, metabolic, and molecular features of pathogens 700 and their hosts [1]. The QTL and associated candidate genes and variants we have identified em- 701 phasize the genetic and functional complexity of virulence traits. For example, the R/C $8^{\mathrm{XL} 280 \mathrm{a}}$ allele 702 we identified is associated with decreased melanization but increased thermal tolerance, oxidative 703 stress resistance, and capsule size. Despite low levels of melanin, and the loss of a key activa- 704 tor of the cAMP-PKA pathway, the strain XL280 is still virulent in an inhalation infection model of 705 murine cryptococcosis $[137,193]$. One must therefore exercise caution when trying to predict the 706 likely effects of natural variation on virulence potential. This is likely to apply equally to engineered 707 genetic manipulations or the effects of drugs that target particular pathways.

Our findings in the present study may also have implications for clinical treatment of crypto- 709 coccal disease. Outright resistance to antifungals such as amphotericin B is rare in Cryptococcus 710 species [84, 194], but variance in the minimum inhibitory concentrations of antifungals, includ- 711 ing amphotericin B, have been observed within species. Such variation could lead to recurring 712 instances of disease within patients $[49,78,195,196]$. A recent survey of antifungal susceptibil- 713 ity in clinical isolates of $C$. neoformans saw increases across a ten-year period in the minimum 714 inhibitory concentration for both fluconazole and amphotericin B [85]. The QTN (at SSK1 and 715 SSK2) identified in the environmental strain $431 \alpha$ are implicated in sensitivity and increased re- 716 sistance (respectively) to amphotericin B and provide concrete examples of the types of natural 717 genetic variants that are present within Cryptococcus that might underlie differences in response 718 to clinical treatment, depending on the particular lineage(s) that a patient is infected with. Simi- 719 larly, in the case of infections by multiple Cryptococcus strains [48], naturally occurring alleles that 720 decrease sensitivity to antifungals are likely targets for selection.

Current global trends point to both a warming planet and an increase rate of resistance to anti- 722 fungal drugs [1, 197]. Studies like the one presented here, that focus on standing genetic variation 723 within species, may help to predict the complex and evolving landscape of fungal virulence, pro- 724 viding insights into both lineages and genetic variants that are likely to be favored or disfavored as 725 environments and clinical treatment change. 


\section{Materials and methods}

\section{Parental strains, laboratory crosses, and isolation of $F_{1}$ progeny}

As described in Sun et al. [92] the parental strains 431 $\alpha, \mathrm{XL280} \alpha \mathrm{SS}$, and XL280a were used in $\quad 729$ $\alpha-\alpha$ unisexual and $\mathbf{a}-\alpha$ bisexual crosses (S1 Table). The parental strain $431 \alpha$ is a natural C. dene- ${ }_{730}$ oformans isolate with the MAT $\alpha$ allele $[92,138]$. The parental strain XL280 $\alpha S$ is an XL280 strain 731 with an inserted NAT resistance marker in the URA5 gene [141] and is congenic to the parental 732 strain XL280a with the exceptions of the URA5 gene, NAT resistance marker, the MAT locus, and 733 a partial duplication of the left arm of chromosome $10[92,137,141]$. Due to the insertion of the 734 $N A T$ in the URA5 gene of the $\mathrm{XL280} \alpha \mathrm{SS}$ strain, a wild type $\mathrm{XL280} \alpha$ strain was used in phenotyping 735 experiments. Because the strains XL280 $\alpha$ and XL280a are congenic with the exception of the MAT ${ }_{736}$ locus, throughout the manuscript only the XL280a strain is referred to when referencing the XL280 737 background.

As described in [92], both a- $\alpha$ bisexual (XL280a $\times 431 \alpha)$ and $\alpha-\alpha$ unisexual (XL280 $\alpha S S \times \quad{ }_{739}$ 431 $\alpha$ ) matings were carried out and progeny were isolated, yielding 261 and 156 progeny respec- 740 tively. Parental strains and segregants were maintained in $35 \%$ glycerol frozen stocks at $-80^{\circ} \mathrm{C}$ and 741 subcultured from freezer stock to YPD media for experimentation. Between segregants derived 742 unisexually verses bisexual, no significant effect was observed in any of the phenotypes examined 743 here - except for fludioxonil sensitivity (see results) - and their phenotypic values were pooled into 744 a single mapping population for use in QTL mapping.

\section{Sequencing, aligning, and variant calling}

In total, 127 segregants, which included 63 from the $\alpha-\alpha$ unisexual, 61 from the a- $\alpha$ bisexual ${ }_{747}$ crosses, and the 3 parental strains, XL280a, XL280 $\alpha$ SS, and $431 \alpha$, were sequenced as previously 748 described [92, 141]. Raw reads were aligned to an XL280 $\propto$ C. deneoformans reference genome 749 [137] using BWA (v0.7.12-r1039, [198]). Variant calling was carried out using SAMtools (v0.1.19- 750 96b5f2294a, [199]) and FreeBayes (v1.2.0, [200]) resulting in 449,197 bi-allelic single nucleotide 751 polymorphisms (SNPs) and 1,500 bi-allelic insertions and deletions (INDELs) between the parental 752 strains, segregating within the $F_{1}$ segregants. 


\section{Segregant filtering and marker creation}

Each of the 127 segregants were filtered to remove those which exhibited aneuploidy, clonality, 755 or lack of recombination as described in Roth et al. [141]. After applying these filtering criteria, 104756 segregants - composed of 55 progeny from $\alpha-\alpha$ unisexual crosses, 46 progeny from a- $\alpha$ bisexual 757 crosses, and the three progenitor strains - were retained for further analysis.

The 449,197 bi-allelic variants sites were filtered on call rate, read depth, allelic read depth 759 ratio, minor allele frequency, and quality scores. Across the 104 segregants, SNP and INDEL 760 sites were required to have $100 \%$ call rate, greater than $10 \times$ coverage in read depth, an allelic 761 read depth ratio of $80 \%$ (for example, if a SNP site has 10 reads mapping over it, 8 of the 10 reads 762 must support the existence of the SNP), a minor allele frequency of $20 \%$, and a $\log _{10}$ quality score, 763 normalized by read depth, of greater than or equal to 0.75 . A maximum $\log _{10}$ read depth of 4.1 was 764 set to filter out SNPs in regions with repetitive elements. Finally, bi-allelic SNP and INDEL sites 765 within $5 \mathrm{~kb}$ of centromeres and of the ends of the chromosomes were removed as these regions 766 are difficult to sequence, resulting in 92,103 genetic variants. These 92,103 genetic variants were 767 then grouped into haploblocks ("haplotype blocks") based on variants in perfect linkage, in order 768 to reduce the number of markers for analysis in genotype-phenotype association tests [201]. This 769 was done for each chromosome such that every haploblock had at least one segregant with a 770 genotype change between contiguous haploblocks, resulting in 3,108 sites across the segregants. 771 The average size of haploblocks was $5.4 \mathrm{~kb}$ with a minimum size of $4.4 \mathrm{~kb}$ and maximum size of 772 $6.3 \mathrm{~kb}$.

\section{Quantitative growth assays}

Quantitative growth assays were measured using absorbance microplate readers (Tecan Sun- 775 rise). Initially, segregants were arrayed in U-bottom 96 well plates containing $100 \mu$ l of liquid YPD, 776 incubated for two days at $30^{\circ} \mathrm{C}$, and after the addition of glycerol, preserved as frozen stocks at 777 $-80^{\circ} \mathrm{C}$. Plates were stored as frozen stocks and used to start each assay. After two days of growth 778 on YPD solid agar plates, segregants were pinned into $150 \mu \mathrm{l}$ liquid YPD and grown on a plate 779 shaker for two days at $30^{\circ} \mathrm{C}$. Subsequently for each segregant, $1 \times 10^{5}$ cells were transferred into 780 $150 \mu \mathrm{l}$ BD Difco yeast nitrogen base (YNB) buffered to pH 7.0 with 0.165 M MOPS (morpholine- 781 
propanesulfonic acid) buffer (Sigma-Aldrich). For drug treatments, amphotericin B (Sigma-Aldrich) 782 was added to YNB from a stock solution of $100 \mu \mathrm{g} / \mathrm{ml}$ for final drug concentrations of $0.075,0.125,783$ and $0.175 \mu \mathrm{g} / \mathrm{ml}$. Cells were grown in microplate readers for three days at either $30^{\circ}, 37^{\circ}$, or $39^{\circ} \mathrm{C}{ }^{784}$ with no drug and assayed for all possible combinations of temperature by amphotericin B concen- 785 tration except for $39^{\circ} \mathrm{C}$ and $0.175 \mu \mathrm{g} / \mathrm{ml}$ because no strains grew at this combination of temperature 786 and drug stress. Assays were replicated four times. To monitor growth, optical density measure- 787 ments were made at a wavelength of $595 \mathrm{~nm}\left(\mathrm{OD}_{595}\right)$ every 15 minutes. To prevent fogging in the 788 machines, plate lids were pre-treated with a solution of $0.05 \%$ Triton X-100 in $20 \% \mathrm{EtOH}$.

\section{Plate based assays}

For plate based assays using solid agar media, segregants were pinned from liquid YPD media 79 to the appropriate, freshly prepared assay media. To minimize edge effects, no segregants were 792 arrayed in the outer rows and columns of the plates. Instead, a control strain was grown in these 793 positions, either $\mathrm{H} 99 \alpha$ or JEC21 $\alpha$.

To assess melanin production, segregants were grown on chemically defined minimal medium 795 containing L-DOPA (7.6 mM L-asparagine monohydrate, 5.6 mM glucose, 10 mM MgSO4, 0.5 mM 796 3,4-dihydroxy-L-phenylalanine, $0.3 \mathrm{mM}$ thiamine- $\mathrm{HCl}$, and $20 \mathrm{nM}$ biotin) and incubated at $30^{\circ} \mathrm{C}$ for ${ }_{797}$ three days in the dark. After three days plates were scanned on an Epson Expression $10000 \mathrm{XL} 798$ Flatbed Scanner in reflective mode (scanned from below) at $300 \mathrm{dpi}$. The grayscale intensity of 799 each colony, as measured using ImageJ, was used as a proxy for melanization, and the mean 800 across three replicates of these values was utilized in statistical association tests. The average 801 Spearman rank correlation coefficient between replicates values was approximately 0.98 .

To assay for hydrogen peroxide $\left(\mathrm{H}_{2} \mathrm{O}_{2}\right)$ sensitivity, segregants were pinned onto YPD plates ${ }^{803}$ supplemented with $5 \mathrm{mM} \mathrm{H}_{2} \mathrm{O}_{2}$ and incubated at $30^{\circ} \mathrm{C}$. After five days, plates were scanned in 804 the same manner as above. Across four replicates, colony growth was scored manually by two 805 individuals on a scale from 0 to 5. The median score per segregant was used for data analysis. 806 Between replicates, the average Spearman rank correlation coefficient was 0.86 . 807

To examine sensitivity to fludioxonil, segregants were similarly pinned onto YPD plates supple- 808 mented with $100 \mu \mathrm{g} / \mathrm{ml}$ fludioxonil and incubated at $30^{\circ} \mathrm{C}$ for five days. Scanned images of colonies 809 
were scored by two observers on a binary scale (growth or no growth) and this value was used in 810 analysis. This assay was replicated in an additional, larger set of segregants generated using the 811 same parental backgrounds (see below).

To assess the response to osmotic stress in this mapping population, colonies were pinned to 813 YP medium containing $1.0 \mathrm{M} \mathrm{NaCl}$, incubated for three days, and then scanned in transmissive 814 mode. The average grayscale density across three replicates of each colony was used as the 815 growth phenotype. Across replicates the average Spearman rank coefficient was 0.80 .

\section{Capsule induction and imaging}

Overnight cell cultures were resuspended in $9.0 \mathrm{ml} \mathrm{CO}$-independent medium (Gibco) and 818 incubated for three days at $37^{\circ} \mathrm{C}$ with shaking at $150 \mathrm{rpm}$. After incubation, cells were washed and 819 then stained with India ink [202]. Each strain was imaged at least three times on an EVOS M5000 820 Cell Imaging System using a 40X objective. Using ImageJ, total area of the capsule plus cell and cell body only were measured and used to quantify capsule size for approximately 30 cells per segregant. The mean of these values was used in QTL mapping.

\section{Growth curve base-lining and parameter estimation}

For each growth experiment, a blank optical density was calculated from the average optical $\quad 825$ density of wells containing no cells, and this value was subtracted from each well on a per plate ba- 826 sis. The first two time points, representing the first fifteen minutes of data collection, were dropped ${ }_{827}$ from analysis. The next five time points were used to baseline the data by calculating the average 828 optical density of these points (the first 1.5 hours) and subtracting this from the remaining sampled 829 time points. These five baseline points were then set to zero. After blank correction and baselin- 830 ing, individual growth curves were filtered using a median filter [203] with a moving, symmetric 831 window of 25 time points, padding the beginning and end of the time courses with zeros or the final 832 $\mathrm{OD}$, respectively. After base-lining and median filtering, we estimated the area under the growth 833 curve at each time point $n$ as $A U C_{n}=\Delta t \sum_{t=0}^{t=n} O D_{595}$ with $\Delta t=.25$ hours (or 15 minutes). For ${ }_{834}$ each segregant, the median of the $A U C_{n}$ across replicates with respect to time was used in QTL 835 mapping across the experimental conditions. One replicate at the condition of $30^{\circ} \mathrm{C}$ and $0 \mu \mathrm{g} / \mathrm{ml} \quad 836$ 
of amphotericin B was dropped from analysis for 60 of the segregants due to poor initial growth ${ }_{837}$ seen in pre-culture plate. At $30^{\circ} \mathrm{C}$, in the absence of drug, the average Spearman rank correlation 838 coefficient between replicates was 0.82 .

\section{QTL mapping}

For each plate based assay and the 3,108 haploblock test sites, a marker regression frame work ${ }_{841}$ was used to associate genotypes to phenotypes. The genotype of each haploblock was coded as 842 zero if inherited from the XL280a (or XL280 $\alpha$ SS) parental strain, and one if from the parental $431 \alpha \quad{ }_{843}$ strain. The model used in statistical association tests can be summarized as $y=\mu+\beta \mathbf{I}_{c}+\epsilon{ }_{844}$ where $\epsilon$ is the error term, $\mu$ is the average phenotype (i.e. mean intensity, growth score, capsule 845 or cell size), $\mathbf{I}_{c}$ is an indicator variable for genotype, and $\beta$ is the coefficient depicting the effect of ${ }_{846}$ having the genotype of XL280a (or XL280 $\alpha$ SS) or genotype of $431 \alpha$ at the given haploblock. An ${ }_{847}$ estimation of this effect, $\beta$, is given by $\widehat{\beta}=\left(X^{\prime} X\right)^{-1} X^{\prime} y$ [204]. The $-\log _{10}$ ( $p$-value) for each unique ${ }_{848}$ haploblock, for a given experiment and phenotype was used as the measure of association. The 849 $95 \%$ confidence intervals for melanization, capsule size, and thermal tolerance were calculated as 850 described in Visscher et al. [205], sampling a 1,000 times with replacement and taking the mean 851 location per maximum haploblock.

For experiments that generated colony growth curves, a function-valued, marker-regression 853 approach was employed to quantify the relationship between genotype and growth phenotypes for 854 each variable haploblock across the 72-hour time course. For these experiments, the area under 855 the growth curve $(A U C)$ was calculated at 15-minute intervals and used as the growth phenotype. 856 As described above, the usual marker-regression model is $y=\mu+\beta \mathbf{I}_{c}+\epsilon$, with $y=A U C$. Across 857 time, the $A U C_{t}$ can be calculated for a given time point within the 72-hour time course and treated 858 as separate phenotypes (Fig 4). This marker-regression model may then be extended for the 859 functional phenotype dependent on time, $y(t)$, where, $y(t)=\mu(t)+\beta(t) \mathbf{I}_{c}+\epsilon(t)$. An estimate of 860 the QTL effect across time is then given by $\widehat{\beta}=\left(X^{\prime} X\right)^{-1} X^{\prime} Y$ where $Y$ is a matrix of segregant ${ }_{861}$ phenotypes with columns that represent the multiple time points [134, 206]. 


\section{Permutation tests}

Permutation tests, as described in Churchill and Doerge [142], were conducted to establish 864 significance thresholds for the $-\log _{10}(\mathrm{p}$-value) from QTL mapping. The number of permutations 865 for all phenotypes analyzed here was 10,000. The same set of random reassignments of genotype 866 to phenotype were used for the eleven temperature by amphotericin B experimental conditions ${ }_{867}$ to conserve correlation structure between the experimental conditions. Each growth curve was 86 treated as a single phenotypic measure during permutations to preserve autocorrelation across 869 time points, and the significance thresholds for the maximum and mean associations between 870 genotype and phenotype (with respect to time) were calculated [134]. The $95^{\text {th }}$ and $99^{\text {th }}$ percentile 871 of the permuted distribution of genotype-phenotype associations was used to estimate thresholds 872 for significance.

\section{Analyzing three-way epistasis}

We used an information-theoretic approach proposed by Hu et al. [146] to evaluate models of 875 three-way genetic epistasis for the association of hydrogen peroxide resistance with genotypic vari- 876 ation at SSK1, SSK2, and RIC8. Hu et al.'s method is based on a measure of the relative amount 877 of "information gain" (IG) attributable to synergistic epistatic interactions, quantified in terms of 878 standard information theoretic measures of mutual information. To apply this approach we dis- 879 cretized each segregant's $\mathrm{H}_{2} \mathrm{O}_{2}$ response as sensitive, intermediate, or resistant based on the 880 observed growth scores, and estimated Hu et al.'s IG statistic using mutual information functions 881 implemented in the Python package scikit-learn [207]. We used permutation tests to simulate null 882 distributions and estimate $p$-values for the IG statistic. For single locus effects, we used permuta- ${ }_{883}$ tions which randomized the relationship between phenotype and genotype at each of the three loci, $\quad 884$ while maintaining the genotypic covariance between loci. For second- and third-order effects we 885 permuted genotypes of samples within each phenotypic class, preserving the independent main 886 effects while randomizing any non-linear interactions, as recommended in Hu et al. [146]. A thou- ${ }_{887}$ sand permutations were used to simulate the distributions of both main and higher order effects. $\quad 888$ We report normalized information gain, expressed as a percentage of the Shannon entropy of the ${ }_{889}$ phenotypic distribution. 
To predict the effects of genetic variants identified between the XL280a (or XL280 $\alpha$ SS) and 892 $431 \alpha$ parental strains, annotated gene features were derived from the C. deneoformans reference 893 strain, JEC21 $\alpha$ [208]. These sequences were then aligned via the blast-like alignment tool (BLAT, 894 [209]) to the $X L 280 \alpha$ reference genome [137]. Per gene, alignments were filtered for sequence 895 identity of $95 \%$ and at most two mismatches between the JEC21 $\alpha$ and XL280 $\alpha$ genomes. There 896 are a total of 5,210 annotated features in the JEC21 $\alpha$ genome annotation, of which 4,800 mapped ${ }_{897}$ perfectly and uniquely to the XL280 $\alpha$ genome. After mapping orthologous genes, the effects of ${ }_{898}$ genetic differences between the XL280a (or XL280 $\alpha$ ) and the $431 \alpha$ backgrounds were imputed ${ }_{899}$ with respect to the predicted exonic and intronic regions.

\section{Gene disruption}

TRACE (Transient CRISPR-Cas9 Coupled with Electroporation [56]) was used to genetically 902 disrupt SSK1 in the parental strains. Deletion constructs were assembled with two-step PCR using 903 homologous arms one $\mathrm{kb}$ in length and a NAT marker, a dominant drug-resistance marker con- 904 ferring resistance to nourseothricin. Single-guide RNAs were designed with Eukaryotic Pathogen 905 CRISPR guide RNA/DNA Design Tool (http://grna. ctegd.uga.edu/) using default parameters, 906 and the gRNA scaffold was amplified from pDD162. To generate complete gRNAs, a one-step 907 overhang PCR was used to amplify the construct from sgRNA, the scaffold, and the U6 promoter 908 (JEC21 $\alpha$ ), following TRACE protocols. Cas9 was amplified from pXL1-Cas9-HygB.

Parental strains were transformed with the amplified constructs following the protocol for elec- 910 troporation in Fan and Lin [56], except competent yeast cells were washed and resuspended in 911 $1 \mathrm{M}$ sorbitol before transformation. Electroporated cells recovered for two hours in YPD before 912 being plated onto YPD supplemented with nourseothricin (YPD+NAT) selective media. After re- 913 streaking, transformants were screened with internal SSK1 primers. Colonies that were capable 914 of growing on YPD+NAT selective media were subsequently screened with external primers for 915 product size, primers that spanned across the gene boundaries, and primers for detecting the 916 presence of Cas9. One ssk 1 transformant for $431 \alpha$ and three ssk1 transformants for XL280a were 917 identified. The inserted deletion construct for each of these transformants was sequenced in full 918 
for confirmation. Primers are listed in S2 Table. To assess gene disruption effects, growth curves 919 for the ssk1 strains were measured at $30^{\circ} \mathrm{C}$ in $0.125 \mu \mathrm{g} / \mathrm{ml}$ amphotericin $\mathrm{B}$, the condition with the 920 largest strength in association at the chromosome 2 QTL which contains the gene SSK1.

\section{Additional fine-mapped crosses, segregant isolation, and sequencing}

To further investigate the identified QTL on chromosome 2 (approximately 150-kb wide), fine- ${ }_{923}$ mapping techniques were applied to generate additional progeny. Parental strains were trans- 924 formed as described previously [56] using the NAT and NEO selectable markers inserted at inter- ${ }_{925}$ genic regions flanking the QTL. The chosen intergenic regions were between genes CNB02680 926 and CNB02690 at approximately 797,055 - 797,281 kb on the left and between genes CNB03490 927 and CNB03500 at approximately 1,047,138 - 1,047,346 kb on the right of the chromosome 2 QTL 928 for the NAT and NEO markers respectively. Transformants were screened as described previously 929 [56] and one strain transformed from the XL280 strain background, with NAT cassette and three 930 strains transformed from the $431 \alpha$ strain background, each with the NEO cassette, were identified (S1 Table). Southern blot probing for the selectable markers was used to determine that only one copy was inserted in the genome for each transformant.

To generate recombinant progeny from the transformed parental strains, spores from mass matings were purified through Percoll gradient centrifugation [22, 210]. Purified, recombinant 935 spores were selected for by growing progeny on YPD+NAT+NEO, and $N A T^{R} N E O^{R}$ segregants 936 were verified as recombinant by colony PCR. In total, 192 progeny were sequenced on a No- 937 vaSeq 6000 (SP) flow cell (150 bp PE). Sequencing data were analyzed in the same manner as 938 described previously. Segregants were filtered to remove clones and progeny with diploid or ane- 939 uploid genomes and 173 segregants were retained for analysis.

\section{Data availability and software}

Raw sequence reads generated from samples utilized in this study are available on NCBl's se- ${ }_{942}$ quence read archive under BioProject identification number PRJNA420966, with individual acces- 943 sion numbers SRR6352893 - SRR6352999, SRR10810110 - SRR10810130, and SRR10861770 944 - SRR10861961. The generated variant call file from the aligned sequenced reads along with 945 
the software developed for both analysis and figure generation are publicly available on GitHub: 946 https://github.com/magwenelab/crypto-QTL-paper.

\section{Acknowledgements}

This work was supported by NIH grant RO1 Al133654 and the GCB Summer Scholars program 949 in Genome Sciences and Medicine (NIH R25) awarded to AS. We would also like to thank our 950 collaborators for their comments on the manuscript: Magwene lab members Thomas Sauters and 951 Lydia Hendrick, members of the Heitman lab, Shelby Priest and Dr. Marcia David Palma (Ph.D) 952 along with Dr. David Tobin (Ph.D) and Tobin lab member, Jared Brewer.

\section{Author contributions}

PM, JH, SS, CF, DM, and CR designed experiments. SS, AA, and CF provided strains and 955 materials. CF, AS, and DM conducted experiments and generated data. PM and CR analyzed 956 the data and CR created figures. PM, DM, and CR wrote the manuscript. JH, SS, AA, and CF 957 provided edits to the manuscript.

\section{Conflicts of interest}

\section{References}

[1] Casadevall A, Fang FC, Pirofski LA. Microbial virulence as an emergent property: con- 962 sequences and opportunities. PLoS Pathogens. 2011;7(7):e1002136. doi:10.1371/jour- 963 nal.ppat. 1002136.

[2] Fisher MC, Henk DA, Briggs CJ, Brownstein JS, Madoff LC, McCraw SL, et al. Emerging 965 fungal threats to animal, plant and ecosystem health. Nature. 2012;484(7393):186-194.

[3] Kim K, Harvell CD. The rise and fall of a six-year coral-fungal epizootic. the American 967 Naturalist. 2004;164(S5):S52-S63.

[4] Gargas A, Trest M, Christensen M, Volk TJ, Blehert DS. Geomyces destructans sp. nov. 969 associated with bat white-nose syndrome. Mycotaxon. 2009;108(1):147-154. 
[5] Fisher MC, Garner TW, Walker SF. Global emergence of Batrachochytrium dendrobatidis 971 and amphibian chytridiomycosis in space, time, and host. Annual review of microbiology. 2009;63:291-310.

[6] Frick WF, Pollock JF, Hicks AC, Langwig KE, Reynolds DS, Turner GG, et al. An emerg- 974 ing disease causes regional population collapse of a common North American bat species. Science. 2010;329(5992):679-682.

[7] Cameron SA, Lozier JD, Strange JP, Koch JB, Cordes N, Solter LF, et al. Patterns of 977 widespread decline in North American bumble bees. Proceedings of the National Academy 978 of Sciences. 2011;108(2):662-667.

[8] Figueroa M, Hammond-Kosack KE, Solomon PS. A review of wheat diseases-a field perspective. Molecular plant pathology. 2018;19(6):1523-1536.

[9] Dietzel K, Valle D, Fierer N, U'Ren JM, Barberán A. Geographical distribution of fungal plant 982 pathogens in dust across the United States. Frontiers in Ecology and Evolution. 2019;7:304.

[10] Byrnes III EJ, Li W, Lewit Y, Ma H, Voelz K, Ren P, et al. Emergence and pathogenicity of highly virulent Cryptococcus gattii genotypes in the northwest United States. PLoS pathogens. 2010;6(4).

[11] Byrnes III EJ, Bartlett KH, Perfect JR, Heitman J. Cryptococcus gattii: an emerging fungal ${ }_{987}$ pathogen infecting humans and animals. Microbes and infection. 2011;13(11):895-907. 988

[12] Byrnes III EJ, Marr KA. The outbreak of Cryptococcus gattii in western North America: 989 epidemiology and clinical issues. Current infectious disease reports. 2011;13(3):256-261. 990

[13] Brown GD, Denning DW, Gow NAR, Levitz SM, Netea MG, White TC. Hidden killers: human 991 fungal infections. Science Translational Medicine. 2012;4(165):165rv13. doi:10.1126/sci- 992 transImed.3004404.

[14] Crawford A, Wilson D. Essential metals at the host-pathogen interface: nutritional im- 994 munity and micronutrient assimilation by human fungal pathogens. FEMS yeast research. 995 2015;15(7).

[15] Komalapriya C, Kaloriti D, Tillmann AT, Yin Z, Herrero-de Dios C, Jacobsen MD, et al. Inte- 997 grative model of oxidative stress adaptation in the fungal pathogen Candida albicans. PLoS One. 2015;10(9).

[16] Jimenez-Lopez C, Lorenz MC. Fungal immune evasion in a model host-pathogen interac- 1000 tion: Candida albicans versus macrophages. PLoS pathogens. 2013;9(11).

[17] Hernández-Chávez MJ, Pérez-García LA, Niño-Vega GA, Mora-Montes HM. Fungal strate- 1002 gies to evade the host immune recognition. Journal of Fungi. 2017;3(4):51.

[18] Navarro-García F, Sánchez M, Nombela C, Pla J. Virulence genes in the pathogenic yeast ${ }_{1004}$ Candida albicans. FEMS microbiology reviews. 2001;25(2):245-268.

[19] Hull CM, Heitman J. Genetics of Cryptococcus neoformans. Annual Review of Genetics. 1006 
[20] Casadevall A. Cards of virulence and the global virulome for humans. Microbe Magazine. 1008 2006;1(8):359.

[21] Liu OW, Chun CD, Chow ED, Chen C, Madhani HD, Noble SM. Systematic genetic 1010 analysis of virulence in the human fungal pathogen Cryptococcus neoformans. Cell. ${ }_{1011}$ 2008;135(1):174-188.

[22] Botts MR, Giles SS, Gates MA, Kozel TR, Hull CM. Isolation and characterization of Cryp- 1013 tococcus neoformans spores reveal a critical role for capsule biosynthesis genes in spore 1014 biogenesis. Eukaryotic Cell. 2009;8(4):595-605.

[23] Saputo S, Chabrier-Rosello Y, Luca FC, Kumar A, Krysan DJ. The RAM network in 1016 pathogenic fungi. Eukaryotic cell. 2012;11(6):708-717.

[24] Bahn YS, Jung KW. Stress signaling pathways for the pathogenicity of Cryptococcus. Eu- 1018 karyotic Cell. 2013;12(12):1564-1577.

[25] O'Meara TR, Xu W, Selvig KM, O'Meara MJ, Mitchell AP, Alspaugh JA. The Cryptococcus 1020 neoformans Rim101 transcription factor directly regulates genes required for adaptation to 1021 the host. Molecular and Cellular Biology. 2014;34(4):673-684.

[26] Brown AJ, Budge S, Kaloriti D, Tillmann A, Jacobsen MD, Yin Z, et al. Stress adaptation in 1023 a pathogenic fungus. Journal of Experimental Biology. 2014;217(1):144-155.

[27] Bultman KM, Kowalski CH, Cramer RA. Aspergillus fumigatus virulence through the lens of 1025 transcription factors. Medical mycology. 2016; p. myw120.

[28] Zaragoza O. Basic principles of the virulence of Cryptococcus. Virulence. 1027 2019;10(1):490-501.

[29] de Castro PA, Colabardini AC, Manfiolli AO, Chiaratto J, Silva LP, Mattos EC, et al. ${ }_{1029}$ Aspergillus fumigatus calcium-responsive transcription factors regulate cell wall architec- 1030 ture promoting stress tolerance, virulence and caspofungin resistance. PLoS Genetics. 1031 2019;15(12).

[30] Hu G, Liu I, Sham A, Stajich JE, Dietrich FS, Kronstad JW. Comparative hybridization reveals 1033 extensive genome variation in the AIDS-associated pathogen Cryptococcus neoformans. ${ }^{1034}$ Genome biology. 2008;9(2):R41.

[31] Fedorova ND, Khaldi N, Joardar VS, Maiti R, Amedeo P, Anderson MJ, et al. Genomic is- 1036 lands in the pathogenic filamentous fungus Aspergillus fumigatus. PLoS genetics. 2008;4(4). 1037

[32] Butler G, Rasmussen MD, Lin MF, Santos MA, Sakthikumar S, Munro CA, et al. Evo- 1038 lution of pathogenicity and sexual reproduction in eight Candida genomes. Nature. ${ }_{1039}$ 2009;459(7247):657-662.

[33] Ngamskulrungroj P, Gilgado F, Faganello J, Litvintseva AP, Leal AL, Tsui KM, et al. Genetic ${ }_{1041}$ diversity of the Cryptococcus species complex suggests that Cryptococcus gattii deserves 1042 
to have varieties. PloS one. 2009;4(6).

[34] D'Souza C, Kronstad J, Taylor G, Warren R, Yuen M, Hu G, et al. Genome varia- 1044 tion in Cryptococcus gattii, an emerging pathogen of immunocompetent hosts. mBio. 1045 2011;2(1):e00342-10.

[35] Hirakawa MP, Martinez DA, Sakthikumar S, Anderson MZ, Berlin A, Gujja S, et al. Ge- 1047 netic and phenotypic intra-species variation in Candida albicans. Genome research. 1048 2015;25(3):413-425.

[36] Mixão V, Perez-Hansen A, Saus E, Boekhout T, Lass-Flörl C, Gabaldón T. Whole-genome 1050 sequencing of the opportunistic yeast pathogen Candida inconspicua uncovers its hybrid ${ }_{1051}$ origin. Frontiers in genetics. 2019;10:383.

[37] Ormerod KL, Morrow CA, Chow EW, Lee IR, Arras SD, Schirra HJ, et al. Comparative ge- 1053 nomics of serial isolates of Cryptococcus neoformans reveals gene associated with carbon ${ }_{1054}$ utilization and virulence. G3: Genes, Genomes, Genetics. 2013;3(4):675-686.

[38] Janbon G, Ormerod KL, Paulet D, Byrnes III EJ, Yadav V, Chatterjee G, et al. Analysis 1056 of the genome and transcriptome of Cryptococcus neoformans var. grubii reveals com- ${ }_{1057}$ plex RNA expression and microevolution leading to virulence attenuation. PLoS Genetics. ${ }_{1058}$ 2014;10(4):e1004261.

[39] Rajasingham R, Smith RM, Park BJ, Jarvis JN, Govender NP, Chiller TM, et al. Global 1060 burden of disease of HIV-associated cryptococcal meningitis: an updated analysis. The ${ }_{1061}$ Lancet Infectious Diseases. 2017;17(8):873-81.

[40] Singh N, Alexander B, Gupta K. Characteristics and outcome of Cryptococcus neoformans 1063 infection of the central nervous system in organ transplant recipients: a prospective, multi- ${ }_{1064}$ center study. 42nd ICAAC, 392. San Diego, CA. 2002;.

[41] Litvintseva AP, Thakur R, Reller LB, Mitchell TG. Prevalence of clinical isolates of Crypto- 1066 coccus gattii serotype $\mathrm{C}$ among patients with AIDS in Sub-Saharan Africa. The Journal of ${ }_{1067}$ infectious diseases. 2005;192(5):888-892.

[42] Fox DL, Müller NL. Pulmonary cryptococcosis in immunocompetent patients: CT findings in 1069 12 patients. American Journal of Roentgenology. 2005;185(3):622-626.

[43] Park BJ, Wannemuehler KA, Marston BJ, Govender N, Pappas PG, Chiller TM. Estimation 1071 of the current global burden of cryptococcal meningitis among persons living with HIV/AIDS. 1072 AIDS. 2009;23(4):525-30.

[44] Fisher JF, Valencia-Rey PA, Davis WB. Pulmonary cryptococcosis in the immunocompe- 1074 tent patient-many questions, some answers. Open Forum Infectious Diseases. 2016;3(3). 1075 doi:10.1093/ofid/ofw167.

[45] Williamson PR, Jarvis JN, Panackal AA, Fisher MC, Molloy SF, Loyse A, et al. Cryptococcal 1077 meningitis: epidemiology, immunology, diagnosis and therapy. Nature Reviews Neurology. 1078 2017;13(1):13. 
[46] Mourad A, Perfect JR. The war on cryptococcosis: a review of the antifungal arsenal. 1080 Memórias do Instituto Oswaldo Cruz. 2018;113(7).

[47] Xu J, Vilgalys R, Mitchell TG. Multiple gene genealogies reveal recent dispersion and hy- 1082 bridization in the human pathogenic fungus Cryptococcus neoformans. Molecular Ecology. 1083 2000;9(10):1471-1481. doi:10.1046/j.1365-294x.2000.01021.x.

[48] Desnos-Ollivier M, Patel S, Spaulding AR, Charlier C, Garcia-Hermoso D, Nielsen K, et al. 1085 Mixed infections and In Vivo evolution in the human fungal pathogen Cryptococcus neofor- ${ }_{1086}$ mans. mBio. 2010;1(1):e00091-10. doi:10.1128/mBio.00091-10.

[49] Hagen F, Illnait-Zaragozí MT, Meis JF, Chew WHM, Curfs-Breuker I, Mouton JW, et al. 1088 Extensive genetic diversity within the Dutch clinical Cryptococcus neoformans population. 1089 Journal of Clinical Microbiology. 2012;50(6):1918-26.

[50] Hagen F, Khayhan K, Theelen B, Kolecka A, Polacheck I, Sionov E, et al. Recognition of 1091 seven species in the Cryptococcus gattii/Cryptococcus neoformans species complex. Fun- 1092 gal Genetics \& Biology. 2015;78:16-48.

[51] Kwon-Chung KJ. A new species of Filobasidiella, the sexual state of Cryptococcus neofor- 1094 mans B and C serotypes. Mycologia. 1976;68(4):943-46.

[52] Marra RE, Huang JC, Fung E, Nielsen K, Heitman J, Vilgalys R, et al. A genetic linkage map 1096 of Cryptococcus neoformans variety neoformans serotype D (Filobasidiella neoformans). 1097 Genetics. 2004;167(2):619-31.

[53] Toffaletti DL, Rude TH, Johnston SA, Durack D, Perfect J. Gene transfer in Cryp- 1099 tococcus neoformans by use of biolistic delivery of DNA. Journal of bacteriology. 1100 1993;175(5):1405-1411.

[54] Goins CL, Gerik KJ, Lodge JK. Improvements to gene deletion in the fungal pathogen Cryp- 1102 tococcus neoformans: absence of Ku proteins increases homologous recombination, and ${ }_{1103}$ co-transformation of independent DNA molecules allows rapid complementation of deletion ${ }_{1104}$ phenotypes. Fungal genetics \& Biology. 2006;43(8):531-544.

[55] Wang Y, Wei D, Zhu X, Pan J, Zhang P, Huo L, et al. A 'suicide' CRISPR-Cas9 system 1106 to promote gene deletion and restoration by electroporation in Cryptococcus neoformans. 1107 Scientific Reports. 2016;6:31145.

[56] Fan Y, Lin X. Multiple applications of a transient CRISPR-Cas9 coupled with electro- ${ }_{1109}$ poration (TRACE) system in the Cryptococcus neoformans species complex. Genetics. 1110 2018;208(4):1357-1372.

[57] Kwon-Chung K, Rhodes J. Encapsulation and melanin formation as indicators of virulence 1112 in Cryptococcus neoformans. Infection \& Immunity. 1986;51(1):218-223.

[58] Mednick AJ, Nosanchuk JD, Casadevall A. Melanization of Cryptococcus neoformans af- 1114 fects lung inflammatory responses during cryptococcal infection. Infection \& Immunity. 1115 2005;73(4):2012-2019. 
[59] Rodrigues ML, Nakayasu ES, Oliveira DL, Nimrichter L, Nosanchuk JD, Almeida IC, et al. ${ }_{1117}$ Extracellular vesicles produced by Cryptococcus neoformans contain protein components 1118 associated with virulence. Eukaryotic Cell. 2008;7(1):58-67.

[60] Ma H, May RC. Virulence in Cryptococcus species. Advances in Applied Microbiology. 1120 2009;67:131-190.

[61] O'Meara TR, Alspaugh JA. The Cryptococcus neoformans capsule: a sword and a shield. ${ }_{1122}$ Clinical microbiology reviews. 2012;25(3):387-408.

[62] Coelho C, Bocca AL, Casadevall A. The tools for virulence of Cryptococcus neoformans. ${ }_{1124}$ Advances in Applied Microbiology. 2014;87:1-41.

[63] Alspaugh JA. Virulence mechanisms and Cryptococcus neoformans pathogenesis. Fungal 1126 Genetics \& Biology. 2015;78:55-58.

[64] Findley K, Rodriguez-Carres M, Metin B, Kroiss J, Fonseca A, Vilgalys R, et al. Phylogeny 1128 and phenotypic characterization of pathogenic Cryptococcus species and closely related 1129 saprobic taxa in the Tremellales. Eukaryotic Cell. 2009;8(3):353-61. doi:10.1128/EC.00373- ${ }_{1130}$ 08.

[65] Casadevall A, Perfect JR. Cryptococcus neoformans. vol. 595. Citeseer; 1998.

[66] Casadevall A, Rosas AL, Nosanchuk JD. Melanin and virulence in Cryptococcus neofor- ${ }_{1133}$ mans. Current Opinion in Microbiology. 2000;3(4):354-358.

[67] Casadevall A, Pirofski La. Accidental virulence, cryptic pathogenesis, martians, lost hosts, 1135 and the pathogenicity of environmental microbes. Eukaryotic cell. 2007;6(12):2169-2174. $\quad 1136$

[68] Rosas AL, Casadevall A. Melanization affects susceptibility of Cryptococcus neoformans ${ }_{1137}$ to heat and cold. FEMS Microbiology Letters. 1997;153(2):265-272. doi:10.1111/j.1574- ${ }_{1138}$ 6968.1997.tb12584.x.

[69] Dadachova E, Bryan RA, Huang X, Moadel T, Schweitzer AD, Aisen P, et al. Ionizing radi- 1140 ation changes the electronic properties of melanin and enhances the growth of melanized ${ }_{1141}$ fungi. PloS one. 2007;2(5).

[70] Jacobson ES, Tinnell S. Antioxidant function of fungal melanin. Journal of bacteriology. ${ }_{1143}$ 1993;175(21):7102-7104.

[71] Alvarez M, Casadevall A. Phagosome extrusion and host-cell survival after Cryptococcus 1145 neoformans phagocytosis by macrophages. Current Biology. 2006;16(21):2161-2165. 1146

[72] Steenbergen J, Shuman H, Casadevall A. Cryptococcus neoformans interactions 1147 with amoebae suggest an explanation for its virulence and intracellular pathogenic ${ }_{1148}$ strategy in macrophages. Proceedings of the National Academy of Sciences. 1149 2001;98(26):15245-15250.

[73] Zaragoza O, Chrisman CJ, Castelli MV, Frases S, Cuenca-Estrella M, Rodríguez-Tudela 1151 $\mathrm{JL}$, et al. Capsule enlargement in Cryptococcus neoformans confers resistance to ox- 1152 
idative stress suggesting a mechanism for intracellular survival. Cellular microbiology. ${ }_{1153}$ 2008;10(10):2043-2057.

[74] Murphy A, Kavanagh K. Emergence of Saccharomyces cerevisiae as a human pathogen: ${ }_{1155}$ implications for biotechnology. Enzyme and Microbial Technology. 1999;25(7):551-557. 1156

[75] Bhabhra R, Miley MD, Mylonakis E, Boettner D, Fortwendel J, Panepinto JC, et al. Disruption ${ }_{1157}$ of the Aspergillus fumigatus gene encoding nucleolar protein CgrA impairs thermotolerant ${ }_{1158}$ growth and reduces virulence. Infection \& Immunity. 2004;72(8):4731-4740.

[76] Perfect JR. Cryptococcus neoformans: the yeast that likes it hot. FEMS Yeast Res. 1160 2006;6(4):463-8. doi:10.1111/j.1567-1364.2006.00051.x.

[77] Strope PK, Skelly DA, Kozmin SG, Mahadevan G, Stone EA, Magwene PM, et al. The 1162 100-genomes strains, an S. cerevisiae resource that illuminates its natural phenotypic and ${ }_{1163}$ genotypic variation and emergence as an opportunistic pathogen. Genome Research. ${ }_{1164}$ 2015;25(5):762-774.

[78] Singer L, Meyer W, Firacative C, Thompson G, Samitz E, Sykes J. Antifungal drug suscep- 1166 tibility and phylogenetic diversity among Cryptococcus isolates from dogs and cats in North ${ }_{1167}$ America. Journal of Clinical Microbiology. 2014;52(6):2061-70.

[79] Vogan AA, Khankhet J, Samarasinghe H, Xu J. Identification of QTLs Associated with ${ }_{1169}$ Virulence Related Traits and Drug Resistance in Cryptococcus neoformans. G3: Genes, 1170 Genomes, Genetics. 2016;6(9):2745-59.

[80] Larsen RA, Bauer M, Thomas AM, Graybill JR. Amphotericin B and fluconazole, a potent ${ }_{1172}$ combination therapy for cryptococcal meningitis. Antimicrobial Agents and Chemotherapy. 1173 2004;48(3):985-991. doi:10.1128/AAC.48.3.985-991.2004.

[81] Perfect JR, Cox GM. Drug resistance in Cryptococcus neoformans. Drug Resistance Up- 1175 dates. 1999;2(4):259-269.

[82] Gray KC, Palacios DS, Dailey I, Endo MM, Uno BE, Wilcock BC, et al. Amphotericin primarily ${ }_{1177}$ kills yeast by simply binding ergosterol. Proceedings of the National Academy of Sciences. 1178 2012;109(7):2234-2239.

[83] Anderson TM, Clay MC, Cioffi AG, Diaz KA, Hisao GS, Tuttle MD, et al. Amphotericin 1180 forms an extramembranous and fungicidal sterol sponge. Nature Chemical Biology. 1181 2014;10(5):400.

[84] Kelly SL, Lamb DC, Taylor M, Corran AJ, Baldwin BC, Powderly WG. Resistance to ampho- ${ }_{1183}$ tericin B associated with defective sterol $\Delta^{8 \rightarrow 7}$ isomerase in a Cryptococcus neoformans ${ }_{1184}$ strain from an AIDS patient. FEMS microbiology letters. 1994;122(1-2):39-42.

[85] Smith KD, Achan B, Hullsiek KH, McDonald TR, Okagaki LH, Alhadab AA, et al. Increased ${ }_{1186}$ antifungal drug resistance in clinical isolates of Cryptococcus neoformans in Uganda. Antimi- ${ }_{1187}$ crobial Agents and Chemotherapy. 2015;59(12):7197-7204. doi:10.1128/AAC.01299-15. 1188

[86] Chesdachai S, Rajasingham R, Nicol MR, Meya DB, Bongomin F, Abassi M, et al. Minimum ${ }_{1189}$ 
inhibitory concentration distribution of fluconazole against Cryptococcus species and the 1190 fluconazole exposure prediction model. In: Open forum infectious diseases. vol. 6. Oxford ${ }_{1191}$ University Press US; 2019. p. 369.

[87] Naicker SD, Mpembe RS, Maphanga TG, Zulu TG, Desanto D, Wadula J, et al. Decreasing 1193 fluconazole susceptibility of clinical South African Cryptococcus neoformans isolates over a ${ }_{1194}$ decade. PLoS neglected tropical diseases. 2020;14(3):e0008137.

[88] Barchiesi F, Cogliati M, Esposto MC, Spreghini E, Schimizzi AM, Wickes BL, 1196 et al. Comparative analysis of pathogenicity of Cryptococcus neoformans serotypes 1197 $A, D$ and $A D$ in murine cryptococcosis. Journal of Infection. 2005;51(1):10-16. 1198 doi:https://doi.org/10.1016/j.jinf.2004.07.013.

[89] Litvintseva AP, Mitchell TG. Most environmental isolates of Cryptococcus neoformans var. 1200 grubii (serotype A) are not lethal for mice. Infection \& Immunity. 2009;77(8):3188-3195.

[90] Mukaremera L, McDonald TR, Nielsen JN, Molenaar CJ, Akampurira A, Schutz C, et al. The ${ }_{1202}$ mouse inhalation model of Cryptococcus neoformans infection recapitulates strain virulence 1203 in humans and shows that closely related strains can possess differential virulence. Infection 1204 and immunity. 2019;87(5).

[91] Martinez LR, Garcia-Rivera J, Casadevall A. Cryptococcus neoformans var. neofor- 1206 mans (Serotype D) Strains Are More Susceptible to Heat than C. neoformans var. 1207 grubii (Serotype A) Strains. Journal of Clinical Microbiology. 2001;39(9):3365-3367. 1208 doi:10.1128/JCM.39.9.3365-3367.2001.

[92] Sun S, Billmyre RB, Mieczkowski PA, Heitman J. Unisexual reproduction drives meiotic ${ }_{1210}$ recombination and phenotypic and karyotypic plasticity in Cryptococcus neoformans. PLoS ${ }_{1211}$ Genetics. 2014;10(12):e1004849.

[93] Small JM, Mitchell TG. Strain variation in antiphagocytic activity of capsular ${ }_{1213}$ polysaccharides from Cryptococcus neoformans serotype A. Infection and immunity. 1214 1989;57(12):3751-3756.

[94] Barcellos VA, Martins L, Fontes AC, Reuwsaat JC, Squizani ED, de Sousa Araújo GR, et al. ${ }_{1216}$ Genotypic and phenotypic diversity of Cryptococcus gattii VGII clinical isolates and its impact ${ }_{1217}$ on virulence. Frontiers in microbiology. 2018;9:132.

[95] Samarasinghe H, Aceituno-Caicedo D, Cogliati M, Kwon-Chung KJ, Rickerts V, Velegraki ${ }_{1219}$ A, et al. Genetic factors and genotype-Environment interactions contribute to variation in 1220 melanin production in the fungal pathogen Cryptococcus neoformans. Scientific Reports. 1221 2018;8(1):9824.

[96] Hagen F, Hare Jensen R, Meis JF, Arendrup MC. Molecular epidemiology and in vitro an- ${ }_{1223}$ tifungal susceptibility testing of 108 clinical Cryptococcus neoformans sensu lato and Cryp- ${ }_{1224}$ tococcus gattii sensu lato isolates from Denmark. Mycoses. 2016;59(9):576-584. 1225

[97] Matos T, Meis JF, Hagen F, et al. Molecular characterization and antifungal susceptibil- ${ }_{1226}$ ity testing of sequentially obtained clinical Cryptococcus deneoformans and Cryptococcus 1227 neoformans isolates from Ljubljana, Slovenia. Mycopathologia. 2018;183(2):371-380. $\quad 1228$ 
[98] Lander ES, Botstein D. Mapping mendelian factors underlying quantitative traits using RFLP ${ }_{1229}$ linkage maps. Genetics. 1989;121(1):185-199.

[99] Lynch M, Walsh B. Genetics and Analysis of Quantitative Traits. Sinauer Associates, Inc. ${ }_{1231}$ 1998;

[100] Magwene PM, Willis JH, Kelly JK. The statistics of bulk segregant analysis using next gen- ${ }_{1233}$ eration sequencing. PLoS computational biology. 2011;7(11):e1002255.

[101] Lorenz K, Cohen BA. Small-and large-effect quantitative trait locus interactions underlie ${ }_{1235}$ variation in yeast sporulation efficiency. Genetics. 2012;192(3):1123-1132. 1236

[102] Ehrenreich IM, Bloom J, Torabi N, Wang X, Jia Y, Kruglyak L. Genetic architecture ${ }_{1237}$ of highly complex chemical resistance traits across four yeast strains. PLoS genetics. ${ }^{1238}$ 2012;8(3):e1002570.

[103] Granek JA, Murray D, Kayrkçi Ö, Magwene PM. The genetic architecture of biofilm formation 1240 in a clinical isolate of Saccharomyces cerevisiae. Genetics. 2013;193(2):587-600. 1241

[104] Bhatia A, Yadav A, Zhu C, Gagneur J, Radhakrishnan A, Steinmetz LM, et al. Yeast growth 1242 plasticity is regulated by environment-specific multi-QTL interactions. G3: Genes, Genomes, 1243 Genetics. 2014;4(5):769-777.

[105] Wilkening S, Lin G, Fritsch ES, Tekkedil MM, Anders S, Kuehn R, et al. An evaluation ${ }_{1245}$ of high-throughput approaches to QTL mapping in Saccharomyces cerevisiae. Genetics. 1246 2014;196(3):853-65. doi:10.1534/genetics.113.160291.

[106] Sirr A, Scott AC, Cromie GA, Ludlow CL, Ahyong V, Morgan TS, et al. Natural variation in 1248 SER1 and ENA6 underlie condition-specific growth defects in Saccharomyces cerevisiae. 1249 G3: Genes, Genomes, Genetics. 2018;8(1):239-251.

[107] Lendenmann MH, Croll D, Stewart EL, McDonald BA. Quantitative trait locus mapping of ${ }_{1251}$ melanization in the plant pathogenic fungus Zymoseptoria tritici. G3: Genes, Genomes, 1252 Genetics. 2014;4(12):2519-2533.

[108] Lendenmann MH, Croll D, McDonald BA. QTL mapping of fungicide sensitivity reveals novel ${ }_{1254}$ genes and pleiotropy with melanization in the pathogen Zymoseptoria tritici. Fungal Genetics 1255 and Biology. 2015;80:53-67.

[109] Lendenmann MH, Croll D, Palma-Guerrero J, Stewart EL, McDonald BA. QTL mapping of ${ }_{1257}$ temperature sensitivity reveals candidate genes for thermal adaptation and growth morphol- ${ }_{1258}$ ogy in the plant pathogenic fungus Zymoseptoria tritici. Heredity. 2016;116(4):384-394. 1259

[110] Christians JK, Cheema MS, Vergara IA, Watt CA, Pinto LJ, Chen N, et al. Quantitative trait 1260 locus (QTL) mapping reveals a role for unstudied genes in Aspergillus virulence. PLoS One. 1261 2011;6(4):e19325.

[111] Lin X, Huang JC, Mitchell TG, Heitman J. Virulence attributes and hyphal growth of C. neo- ${ }_{1263}$ formans are quantitative traits and the MAT $\alpha$ allele enhances filamentation. PLoS Genetics. ${ }_{1264}$ 2006;2(11):e187. doi:10.1371/journal.pgen.0020187. 
[112] Vogan AA, Khankhet J, Xu J. Evidence for mitotic recombination within the basidia of a 1266 hybrid cross of Cryptococcus neoformans. PLoS One. 2013;8(5):e62790.

[113] Tjørve KM, Tjørve E. The use of Gompertz models in growth analyses, and new 1268 Gompertz-model approach: An addition to the Unified-Richards family. PloS one. 1269 2017;12(6):e0178691.

[114] Tonner PD, Darnell CL, Engelhardt BE, Schmid AK. Detecting differential growth of microbial 1271 populations with Gaussian process regression. Genome research. 2017;27(2):320-333. 1272

[115] Kirkpatrick M, Heckman N. A quantitative genetic model for growth, shape, reac- ${ }_{1273}$ tion norms, and other infinite-dimensional characters. Journal of mathematical biology. 1274 1989;27(4):429-450.

[116] Kirkpatrick M, Lofsvold D, Bulmer M. Analysis of the inheritance, selection and evolution of ${ }_{1276}$ growth trajectories. Genetics. 1990;124(4):979-993.

[117] Kingsolver JG, Gomulkiewicz R, Carter PA. Variation, selection and evolution of function- 1278 valued traits. In: Microevolution rate, pattern, process. Springer; 2001. p. 87-104.

[118] Ragland G, Carter P. Genetic covariance structure of growth in the salamander Ambystoma 1280 macrodactylum. Heredity. 2004;92(6):569-578.

[119] Griswold CK, Gomulkiewicz R, Heckman N. Hypothesis testing in comparative and experi- 1282 mental studies of function-valued traits. Evolution. 2008;62(5):1229-1242.

[120] Stinchcombe JR, Kirkpatrick M, valued Traits Working Group F, et al. Genetics and evolution ${ }_{1284}$ of function-valued traits: understanding environmentally responsive phenotypes. Trends in ${ }_{1285}$ Ecology \& Evolution. 2012;27(11):637-647.

[121] Gomulkiewicz R, Kingsolver JG, Carter PA, Heckman N. Variation and evolution of function- 1287 valued traits. Annual Review of Ecology, Evolution, and Systematics. 2018;49:139-164. 1288

[122] Ma CX, Casella G, Wu R. Functional mapping of quantitative trait loci underlying the char- ${ }_{1289}$ acter process: a theoretical framework. Genetics. 2002;161(4):1751-1762.

[123] Wu R, Ma CX, Zhao W, Casella G. Functional mapping for quantitative trait loci governing 1291 growth rates: a parametric model. Physiological Genomics. 2003;14(3):241-249.

[124] Yang R, Tian Q, Xu S. Mapping quantitative trait loci for longitudinal traits in line crosses. ${ }_{1293}$ Genetics. 2006;173(4):2339-2356.

[125] Yap JS, Wang C, Wu R. A computational approach for functional mapping of quantitative ${ }_{1295}$ trait loci that regulate thermal performance curves. PLoS One. 2007;2(6).

[126] Li Z, Hallingbäck HR, Abrahamsson S, Fries A, Gull BA, Sillanpää MJ, et al. Functional multi- ${ }_{1297}$ locus QTL mapping of temporal trends in scots pine wood traits. G3: Genes, Genomes, 1298 Genetics. 2014;4(12):2365-2379.

[127] Li R, Jeong K, Davis JT, Kim S, Lee S, Michelmore RW, et al. Integrated QTL and eQTL map- 1300 
ping provides insights and candidate genes for fatty acid composition, flowering time, and 1301 growth traits in a $F_{2}$ population of a novel synthetic allopolyploid Brassica napus. Frontiers ${ }_{1302}$ in plant science. 2018;9:1632.

[128] Camargo AV, Mackay I, Mott R, Han J, Doonan JH, Askew K, et al. Functional mapping of ${ }_{1304}$ quantitative trait loci (QTLS) associated with plant performance in a wheat magic mapping ${ }_{1305}$ population. Frontiers in plant science. 2018;9:887.

[129] VanWallendael A, Bonnette J, Juenger TE, Fritschi FB, Fay PA, Mitchell RB, et al. Geo- 1307 graphic variation in the genetic basis of resistance to leaf rust in locally adapted ecotypes of ${ }_{1308}$ the biofuel crop switchgrass (Panicum virgatum). bioRxiv. 2019; p. 619148.

[130] Yang J, Wu R, Casella G. Nonparametric functional mapping of quantitative trait loci. Bio- 1310 metrics. 2009;65(1):30-39.

[131] Min L, Yang R, Wang X, Wang B. Bayesian analysis for genetic architecture of dynamic 1312 traits. Heredity. 2011;106(1):124-133.

[132] Xiong H, Goulding EH, Carlson EJ, Tecott LH, McCulloch CE, Sen Ś. A flexible estimating 1314 equations approach for mapping function-valued traits. Genetics. 2011;189(1):305-316.

[133] Li Z, Sillanpää MJ. A Bayesian nonparametric approach for mapping dynamic quantitative 1316 traits. Genetics. 2013;194(4):997-1016.

[134] Kwak IY, Moore CR, Spalding EP, Broman KW. A simple regression-based method 1318 to map quantitative trait loci underlying function-valued phenotypes. Genetics. 1319 2014;197(4):1409-1416.

[135] Kwak IY, Moore CR, Spalding EP, Broman KW. Mapping quantitative trait loci underlying 1321 function-valued traits using functional principal component analysis and multi-trait mapping. 1322 G3: Genes, Genomes, Genetics. 2016;6(1):79-86.

[136] Vanhatalo J, Li Z, Sillanpää MJ. A Gaussian process model and Bayesian variable selection ${ }_{1324}$ for mapping function-valued quantitative traits with incomplete phenotypic data. Bioinformat- 1325 ics. 2019;35(19):3684-3692.

[137] Zhai B, Zhu P, Foyle D, Upadhyay S, Idnurm A, Lin X. Congenic strains of the filamen- ${ }_{1327}$ tous form of Cryptococcus neoformans for studies of fungal morphogenesis and virulence. 1328 Infection \& Immunity. 2013;81(7):2626-2637.

[138] Sun S, Hsueh YP, Heitman J. Gene conversion occurs within the mating-type locus of Cryp- ${ }_{1330}$ tococcus neoformans during sexual reproduction. PLoS Genetics. 2012;8(7):e1002810.

[139] Bennett JE, Kwon-Chung KJ, Howard DH. Epidemiologic differences among serotypes 1332 of Cryptococcus neoformans. American Journal of Epidemiology. 1977;105(6):582-586. 1333 doi:10.1093/oxfordjournals.aje.a112423.

[140] Lin X, Litvintseva AP, Nielsen K, Patel S, Floyd A, Mitchell TG, et al. $\alpha$ AD $\alpha$ hybrids of ${ }_{1335}$ Cryptococcus neoformans: evidence of same-sex mating in nature and hybrid fitness. PLoS ${ }_{1336}$ Genetics. 2007;3(10):1975-90. 
[141] Roth C, Sun S, Billmyre RB, Heitman J, Magwene PM. A high-resolution map of meiotic ${ }_{1338}$ recombination in Cryptococcus deneoformans demonstrates decreased recombination in 1339 unisexual reproduction. Genetics. 2018;209(2):567-78.

[142] Churchill GA, Doerge RW. Empirical threshold values for quantitative trait mapping. Genet- ${ }_{1341}$ ics. 1994;138(3):963-71.

[143] Bahn YS, Geunes-Boyer S, Heitman J. Ssk2 mitogen-activated protein kinase kinase kinase ${ }_{1343}$ governs divergent patterns of the stress-activated Hog1 signaling pathway in Cryptococcus 1344 neoformans. Eukaryotic Cell. 2007;6(12):2278-2289.

[144] Gong J, Grodsky JD, Zhang Z, Wang P. A Ric8/synembryn homolog promotes Gpa1 and 1346 Gpa2 activation to respectively regulate cyclic AMP and pheromone signaling in Cryptococ- 1347 cus neoformans. Eukaryotic Cell. 2014;13(10):1290-1299. doi:10.1128/EC.00109-14.

[145] Kilani J, Fillinger S. Phenylpyrroles: 30 years, two molecules and (nearly) no resistance. 1349 Frontiers in Microbiology. 2016;7:2014.

[146] Hu T, Chen Y, Kiralis JW, Collins RL, Wejse C, Sirugo G, et al. An information-gain approach 1351 to detecting three-way epistatic interactions in genetic association studies. Journal of the ${ }_{1352}$ American Medical Informatics Association. 2013;20(4):630-636.

[147] Cover T, Thomas J. Elements of Information Theory. Wiley Interscience; 2006.

[148] Bahn YS, Kojima K, Cox GM, Heitman J. A unique fungal two-component system regu- 1355 lates stress responses, drug sensitivity, sexual development, and virulence of Cryptococcus 1356 neoformans. Molecular Biology of the Cell. 2006;17(7):3122-3135.

[149] Emery HS, Shelburne CP, Bowman JP, Fallon PG, Schulz CA, Jacobson ES. Genetic study 1358 of oxygen resistance and melanization in Cryptococcus neoformans. Infection and immunity. 1359 1994;62(12):5694-5697.

[150] Chaturvedi V, Flynn T, Niehaus WG, Wong B. Stress tolerance and pathogenic potential of ${ }_{1361}$ a mannitol mutant of Cryptococcus neoformans. Microbiology. 1996;142(4):937-943. 1362

[151] Jones TH, McClelland EE, McFeeters H, McFeeters RL. Novel antifungal activity for the 1363 lectin scytovirin: inhibition of Cryptococcus neoformans and Cryptococcus gattii. Frontiers 1364 in microbiology. 2017;8:755.

[152] Hohmann S, Krantz M, Nordlander B. Yeast osmoregulation. In: Methods in Enzymology. 1366 vol. 428. Elsevier; 2007. p. 29-45.

[153] Brewster JL, Gustin MC. Hog1: 20 years of discovery and impact. Sci Signal. 1368 2014;7(343):re7-re7.

[154] Caza M, Kronstad JW. The cAMP/protein kinase A pathway regulates virulence and adap- 1370 tation to host conditions in Cryptococcus neoformans. Frontiers in Cellular and Infection 1371 Microbiology. 2019;9:212. doi:10.3389/fcimb.2019.00212.

[155] Granek JA, Kayikci O, Magwene PM. Pleiotropic signaling pathways orchestrate yeast de- 1373 
velopment. Current Opinion in Microbiology. 2011;14(6):676-681.

[156] Lin CJ, Chen YL. Conserved and divergent functions of the cAMP/PKA signaling pathway 1375 in Candida albicans and Candida tropicalis. Journal of Fungi. 2018;4(2):68.

[157] Day AM, McNiff MM, da Silva Dantas A, Gow NA, Quinn J. Hog1 regulates stress 1377 tolerance and virulence in the emerging fungal pathogen Candida auris. mSphere. 1378 2018;3(5):e00506-18.

[158] Wright SJ, Inchausti R, Eaton CJ, Krystofova S, Borkovich KA. RIC8 is a guanine-nucleotide 1380 exchange factor for $\mathrm{G} \alpha$ subunits that regulates growth and development in Neurospora ${ }_{1381}$ crassa. Genetics. 2011;189(1):165-176.

[159] Li S, Giardina DM, Siegal ML. Control of nongenetic heterogeneity in growth rate and stress 1383 tolerance of Saccharomyces cerevisiae by cyclic AMP-regulated transcription factors. PLoS ${ }_{1384}$ genetics. 2018;14(11):e1007744.

[160] Wang Y, Casadevall A. Susceptibility of melanized and nonmelanized Cryptococ- 1386 cus neoformans to nitrogen-and oxygen-derived oxidants. Infection and Immunity. 1387 1994;62(7):3004-3007.

[161] Hicks JK, D’Souza CA, Cox GM, Heitman J. Cyclic AMP-dependent protein kinase catalytic ${ }_{1389}$ subunits have divergent roles in virulence factor production in two varieties of the fungal 1390 pathogen Cryptococcus neoformans. Eukaryot Cell. 2004;3(1):14-26.

[162] Hicks JK, Heitman J. Divergence of protein kinase A catalytic subunits in Cryptococcus 1392 neoformans and Cryptococcus gattii illustrates evolutionary reconfiguration of a signaling 1393 cascade. Eukaryot Cell. 2007;6(3):413-20.

[163] Halme A, Bumgarner S, Styles C, Fink GR. Genetic and epigenetic regulation of the FLO ${ }_{1395}$ gene family generates cell-surface variation in yeast. Cell. 2004;116(3):405-415.

[164] Roop JI, Brem RB. Rare variants in hypermutable genes underlie common morphology and 1397 growth traits in wild Saccharomyces paradoxus. Genetics. 2013;195(2):513-525.

[165] Taylor MB, Ehrenreich IM. Genetic interactions involving five or more genes contribute to a 1399 complex trait in yeast. PLoS genetics. 2014;10(5):e1004324.

[166] Kohn LM, Anderson JB. The underlying structure of adaptation under strong selection in 121401 experimental yeast populations. Eukaryotic cell. 2014;13(9):1200-1206.

[167] Kayikci O, Magwene PM. Divergent roles for CAMP-PKA signaling in the regulation of fila- 1403 mentous growth in Saccharomyces cerevisiae and Saccharomyces bayanus. G3: Genes, 1404 Genomes, Genetics. 2018;8(11):3529-3538.

[168] Ko YJ, Yu YM, Kim GB, Lee GW, Maeng PJ, Kim S, et al. Remodeling of global transcription 1406 patterns of Cryptococcus neoformans genes mediated by the stress-activated HOG signaling ${ }_{1407}$ pathways. Eukaryotic Cell. 2009;8(8):1197-1217. 
on differentiation and virulence of Cryptococcus neoformans. Molecular Biology of the Cell. 1410 2005;16(5):2285-2300.

[170] Hohmann S. Control of high osmolarity signalling in the yeast Saccharomyces cerevisiae. 1412 FEBS letters. 2009;583(24):4025-4029.

[171] Cramer KL, Gerrald QD, Nichols CB, Price MS, Alspaugh JA. Transcription factor Nrg1 me- 1414 diates capsule formation, stress response, and pathogenesis in Cryptococcus neoformans. 1415 Eukaryotic Cell. 2006;5(7):1147-1156.

[172] Haynes BC, Skowyra ML, Spencer SJ, Gish SR, Williams M, Held EP, et al. Toward ${ }_{1417}$ an integrated model of capsule regulation in Cryptococcus neoformans. PLoS Pathog. 1418 2011;7(12):e1002411.

[173] Gutin J, Sadeh A, Rahat A, Aharoni A, Friedman N. Condition-specific genetic interaction 1420 maps reveal crosstalk between the cAMP/PKA and the HOG MAPK pathways in the activa- ${ }_{1421}$ tion of the general stress response. Molecular systems biology. 2015;11(10):829.

[174] Banerjee D, Bloom AL, Panepinto JC. Opposing PKA and Hog1 signals control the post- ${ }_{1423}$ transcriptional response to glucose availability in Cryptococcus neoformans. Molecular mi- ${ }_{1424}$ crobiology. 2016;102(2):306-320.

[175] Lew RR. Turgor and net ion flux responses to activation of the osmotic MAP kinase cascade 1426 by fludioxonil in the filamentous fungus Neurospora crassa. Fungal Genetics and Biology. 1427 2010;47(8):721-726.

[176] Ochiai N, Fujimura M, Motoyama T, Ichiishi A, Usami R, Horikoshi K, et al. Characterization of ${ }_{1429}$ mutations in the two-component histidine kinase gene that confer fludioxonil resistance and 1430 osmotic sensitivity in the os-1 mutants of Neurospora crassa. Pest Management Science: ${ }_{1431}$ formerly Pesticide Science. 2001;57(5):437-442.

[177] Maeng S, Ko YJ, Kim GB, Jung KW, Floyd A, Heitman J, et al. Comparative transcriptome ${ }_{1433}$ analysis reveals novel roles of the Ras and cyclic AMP signaling pathways in environmental ${ }_{1434}$ stress response and antifungal drug sensitivity in Cryptococcus neoformans. Eukaryotic cell. ${ }_{1435}$ 2010;9(3):360-378.

[178] Alspaugh JA, Perfect JR, Heitman J. Cryptococcus neoformans mating and virulence are ${ }_{1437}$ regulated by the G-protein $\alpha$ subunit GPA1 and cAMP. Genes Dev. 1997;11(23):3206-17. 1438 doi:10.1101/gad.11.23.3206.

[179] Alspaugh JA, Pukkila-Worley R, Harashima T, Cavallo LM, Funnell D, Cox GM, et al. 1440 Adenylyl cyclase functions downstream of the $\mathrm{G} \alpha$ protein Gpa1 and controls mating and ${ }_{1441}$ pathogenicity of Cryptococcus neoformans. Eukaryotic cell. 2002;1(1):75-84.

[180] O'Meara TR, Norton D, Price MS, Hay C, Clements MF, Nichols CB, et al. Interaction of ${ }_{1443}$ Cryptococcus neoformans Rim101 and protein kinase A regulates capsule. PLoS Pathog. 1444 2010;6(2):e1000776. doi:10.1371/journal.ppat.1000776.

[181] Zackrisson M, Hallin J, Ottosson LG, Dahl P, Fernandez-Parada E, Ländström E, et al. ${ }_{1446}$ Scan-o-matic: High-Resolution Microbial Phenomics at a Massive Scale. G3 (Bethesda). ${ }_{1447}$ 
2016;6(9):3003-14. doi:10.1534/g3.116.032342.

[182] Shen G, Whittington A, Song K, Wang P. Pleiotropic function of intersectin homologue Cin1 1449 in Cryptococcus neoformans. Molecular microbiology. 2010;76(3):662-676.

[183] Fang W, Price MS, Toffaletti DL, Tenor J, Betancourt-Quiroz M, Price JL, et al. Pleiotropic ${ }_{1451}$ effects of deubiquitinating enzyme Ubp5 on growth and pathogenesis of Cryptococcus neo- ${ }_{1452}$ formans. PLoS One. 2012;7(6).

[184] Tefsen B, Grijpstra J, Ordonez S, Lammers M, van Die I, De Cock H. Deletion of the CAP10 1454 gene of Cryptococcus neoformans results in a pleiotropic phenotype with changes in expres- ${ }_{1455}$ sion of virulence factors. Research in microbiology. 2014;165(6):399-410.

[185] Cavalheiro M, Pais P, Galocha M, Teixeira MC. Host-pathogen interactions mediated by ${ }_{1457}$ MDR transporters in fungi: as pleiotropic as it gets! Genes. 2018;9(7):332.

[186] So YS, Lee DG, Idnurm A, laniri G, Bahn YS. The TOR Pathway Plays Pleiotropic Roles in 1459 Growth and Stress Responses of the Fungal Pathogen Cryptococcus neoformans. Genetics. 1460 2019;212(4):1241-1258.

[187] Lev S, Li C, Desmarini D, Sorrell TC, Saiardi A, Djordjevic JT. Fungal kinases with a sweet ${ }_{1462}$ tooth: Pleiotropic roles of their phosphorylated inositol sugar products in the pathogenicity ${ }_{1463}$ of Cryptococcus neoformans present novel drug targeting opportunities. Frontiers in cellular ${ }_{1464}$ and infection microbiology. 2019;9:248.

[188] Oliver F, Christians JK, Liu X, Rhind S, Verma V, Davison C, et al. Regulatory Varia- 1466 tion at Glypican-3 Underlies a Major Growth QTL in Mice. PLOS Biology. 2005;3(5):e135. 1467 doi:10.1371/journal.pbio.0030135.

[189] Lee JT, Coradini ALV, Shen A, Ehrenreich IM. Layers of Cryptic Genetic Variation Underlie a 1469 Yeast Complex Trait. Genetics. 2019;211(4):1469-1482. doi:10.1534/genetics.119.301907. 1470

[190] Jakobson CM, Jarosz DF. Molecular Origins of Complex Heritability in Natural Genotype-to- 1471 Phenotype Relationships. Cell Syst. 2019;8(5):363-379.e3. doi:10.1016/j.cels.2019.04.002. 1472

[191] Li Z, Sillanpää MJ. Overview of LASSO-related penalized regression methods for 1473 quantitative trait mapping and genomic selection. Theoretical and applied genetics. 1474 2012;125(3):419-435.

[192] Bloom JS, Boocock J, Treusch S, Sadhu MJ, Day L, Oates-Barker H, et al. Rare variants 1476 contribute disproportionately to quantitative trait variation in yeast. Elife. 2019;8.

[193] Feretzaki M, Hardison SE, Wormley Jr FL, Heitman J. Cryptococcus neoformans hyperfila- 1478 mentous strain is hypervirulent in a murine model of cryptococcal meningoencephalitis. PloS ${ }_{1479}$ one. 2014;9(8):e104432.

[194] Singhal S, Gupta P, Lamba BS, Singh P, Chouhan MI, Meher D. Rare case of ampho- ${ }_{1481}$ tericin B resistant cryptococcal meningitis in HIV non reactive patient. International Journal ${ }_{1482}$ of Infectious Diseases. 2016;45:199-200. doi:https://doi.org/10.1016/j.jid.2016.02.459. 
[195] Govender NP, Patel J, van Wyk M, Chiller TM, Lockhart SR. Trends in antifungal drug sus- ${ }_{1484}$ ceptibility of Cryptococcus neoformans isolates obtained through population-based surveil- ${ }_{1485}$ lance in South Africa in 2002-2003 and 2007-2008. Antimicrobial Agents and Chemotherapy. 1486 2011;55(6):2606-2611. doi:10.1128/AAC.00048-11.

[196] Espinel-Ingroff A, Chowdhary A, Cuenca-Estrella M, Fothergill A, Fuller J, Hagen F, et al. ${ }_{1488}$ Cryptococcus neoformans-Cryptococcus gattii species complex: an international study of ${ }_{1489}$ wild-type susceptibility endpoint distributions and epidemiological cutoff values for ampho- 1490 tericin B and flucytosine. Antimicrobial Agents and Chemotherapy. 2012;56(6):3107-3113. 1491 doi:10.1128/AAC.06252-11.

[197] Fisher MC, Hawkins NJ, Sanglard D, Gurr SJ. Worldwide emergence of resistance to antifun- 1493 gal drugs challenges human health and food security. Science. 2018;360(6390):739-742. 1494

[198] Li H, Durbin R. Fast and accurate short read alignment with Burrows-Wheeler transform. 1495 Bioinformatics. 2009;25(14):1754-1760.

[199] Li H. A statistical framework for SNP calling, mutation discovery, association mapping ${ }_{1497}$ and population genetical parameter estimation from sequencing data. Bioinformatics. 1498 2011;27(21):2987-2993.

[200] Garrison E, Marth G. Haplotype-based variant detection from short-read sequencing. ArXiv 1500 Preprint. 2012; p. arXiv:1207.3907.

[201] Xu S. Genetic mapping and genomic selection using recombination breakpoint data. Ge- 1502 netics. 2013;195(3):1103-15. doi:10.1534/genetics.113.155309.

[202] Perfect JR, Casadevall A. Cryptococcosis. Infectious disease clinics of North America. 1504 2002;16(4):837-74.

[203] Gallagher N, Wise G. A theoretical analysis of the properties of median filters. 1506 IEEE Transactions on Acoustics, Speech, and Signal Processing. 1981;29(6):1136-41. 1507 doi:10.1109/TASSP.1981.1163708.

[204] Friedman J, Hastie T, Tibshirani R. The elements of statistical learning. vol. 1. Springer ${ }_{1509}$ Series in Statistics New York; 2001.

[205] Visscher PM, Thompson R, Haley CS. Confidence intervals in QTL mapping by bootstrap- 1511 ping. Genetics. 1996;143(2):1013-1020.

[206] Ramsay JO, Silverman BW. Functional Data Analysis: Second edition. Springer. 2005;. 1513

[207] Pedregosa F, Varoquaux G, Gramfort A, Michel V, Thirion B, Grisel O, et al. Scikit-learn: 1514 Machine Learning in Python. Journal of Machine Learning Research. 2011;12:2825-2830. 1515

[208] Loftus BJ, Fung E, Roncaglia P, Rowley D, Amedeo P, Bruno D, et al. The genome 1516 of the basidiomycetous yeast and human pathogen Cryptococcus neoformans. Science. 1517 2005;307(5713):1321-24.

[209] Kent WJ. BLAT—-the BLAST-like alignment tool. Genome research. 2002;12(4):656-664. $\quad 1519$ 
bioRxiv preprint doi: https://doi.org/10.1101/2020.08 21.259861: this version posted December 16,2020 . The copyright holder for this preprint (which was not certified by peer review) is the author/funder, who has granted bioRxiv a license to display the preprint in perpetuity. It is made available under aCC-BY-NC-ND 4.0 International license.

[210] Fu C, Heitman J. PRM1 and KAR5 function in cell-cell fusion and karyogamy to drive dis- ${ }_{1520}$ tinct bisexual and unisexual cycles in the Cryptococcus pathogenic species complex. PLoS ${ }_{1521}$ Genetics. 2017;13(11):e1007113.

1522

[211] Kwon-Chung KJ, Edman JC, Wickes BL. Genetic association of mating types and virulence ${ }_{1523}$ in Cryptococcus neoformans. Infection and immunity. 1992;60(2):602-605. 


\section{Supplemental information}

S1 Table. Genotypes of parental and transformant strains. QTL-L and QTL-R refer to intergenic regions on chromosome 2 at 797,055 - 797,281 bp, between genes CNB02680 and CNB02690 and at 1,047,138 - 1,047,346 bp, between genes CNB03490 and CNB03500, respectively.

\begin{tabular}{cclr}
\hline Strain & Name & Genotype & Source \\
\hline SSA837 & $431 \alpha$ & wild type & {$[92]$} \\
SSB830 & XL280a & wild type & {$[211]$} \\
SSA853 & XL280 $\alpha S S$ & MAT $\alpha$, ura5::NAT & {$[92,141]$} \\
PMY2408 & CF1705 & MAT $\alpha, Q T L-R:: N E O(431 \alpha)$ & this study \\
PMY2420 & CF1706 & MAT $\alpha, Q T L-R:: N E O(431 \alpha)$ & this study \\
PMY2432 & CF1707 & MAT $\alpha$, QTL-R::NEO $(431 \alpha)$ & this study \\
PMY2444 & CF1730 & MATa, QTL-L::NAT $(\mathrm{XL280a})$ & this study \\
PMY2552 & $1 a-12$ & MAT $\alpha$, SSK1::NAT $(431 \alpha)$ & this study \\
PMY2553 & $4 b-2$ & MATa, SSK1::NAT $(\mathrm{XL280a})$ & this study \\
PMY2554 & $5 a-8$ & MATa, SSK1::NAT $(\mathrm{XL280a})$ & this study \\
PMY2555 & $5 b-1$ & MATa, SSK1::NAT $(\mathrm{XL280a)}$ & this study \\
\hline
\end{tabular}

\section{S2 Table. Primer sequences used in this study.}

S3 Table. Predicted ORF and summaries of genetic variants within QTL regions. For each gene, the number of genetic variants within and upstream of a predicted gene is provided. For each gene the upstream distance from the 5' UTR was taken as the intergenic distance (maximum 500 $\mathrm{bp}$ ) between flanking genes on the same strand. For those genes containing genetic variants the predicted protein length of the reference strain JEC21 $\alpha$, parental strain XL280a, and the parental strain $431 \alpha$ is listed. The number of predicted stop-codons in the parental strains, non-synonymous changes, and variants within UTRs, exons and introns are also listed. Gene names are given in the C. deneoformans reference strain JEC21 $\alpha$ background [208]. The position and strand are relative to the $\mathrm{XL280} \alpha$ strain [137]. 

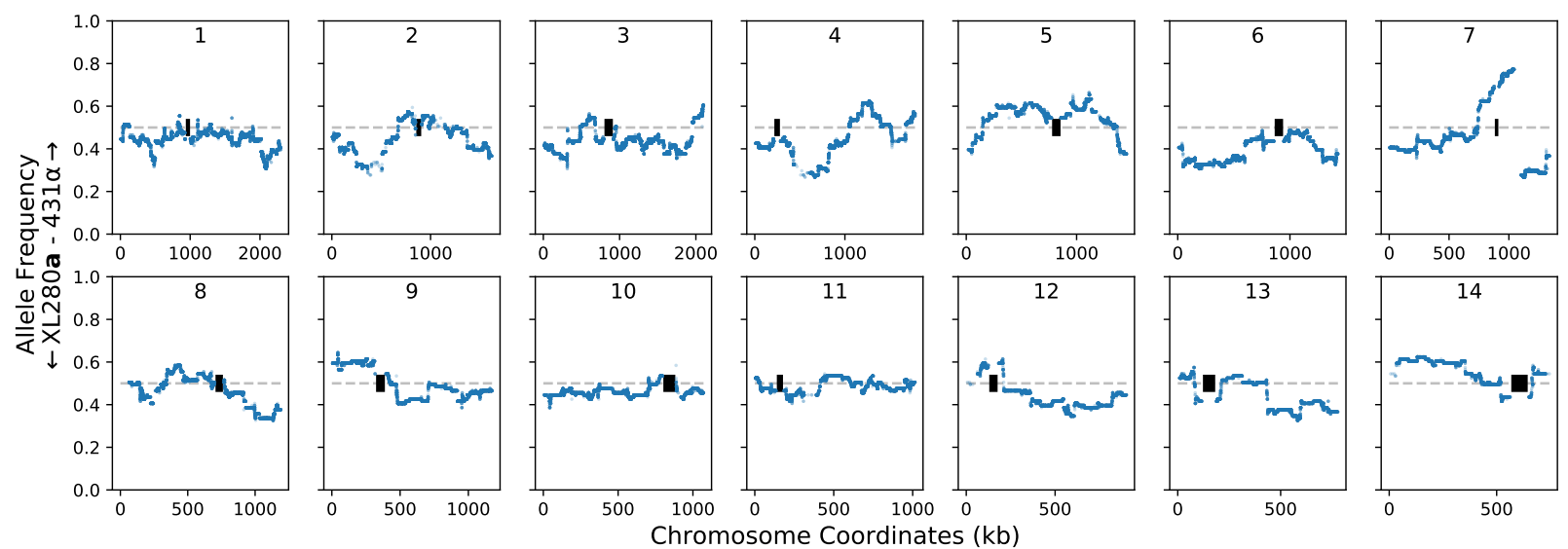

S1 Fig. Genome-wide haplotype allele frequencies. C. deneoformans strains XL280a and XL280 $\alpha$ SS were crossed with $431 \alpha$ in a- $\alpha$ bisexual and $\alpha-\alpha$ unisexual matings, generating 101 segregants. Between the parental strains there are 92,103 bi-allelic genetic variants (see methods) and these genetic variants are collapsed across the segregants, based on genetic exchange events, generating 3,108 unique haplotypes across the genome. The allele frequencies of these haplotypes (blue dots) per chromosome are shown for each of the 14 chromosomes (numbers denote chromosome). A horizontal, grey dashed line marks an allele frequency of 0.5 . Centromere locations are marked by black rectangles. The bias present on the right of chromosome 7 is due to selectable genetic markers used to generate progeny from the $\alpha-\alpha$ unisexual cross [141]. 

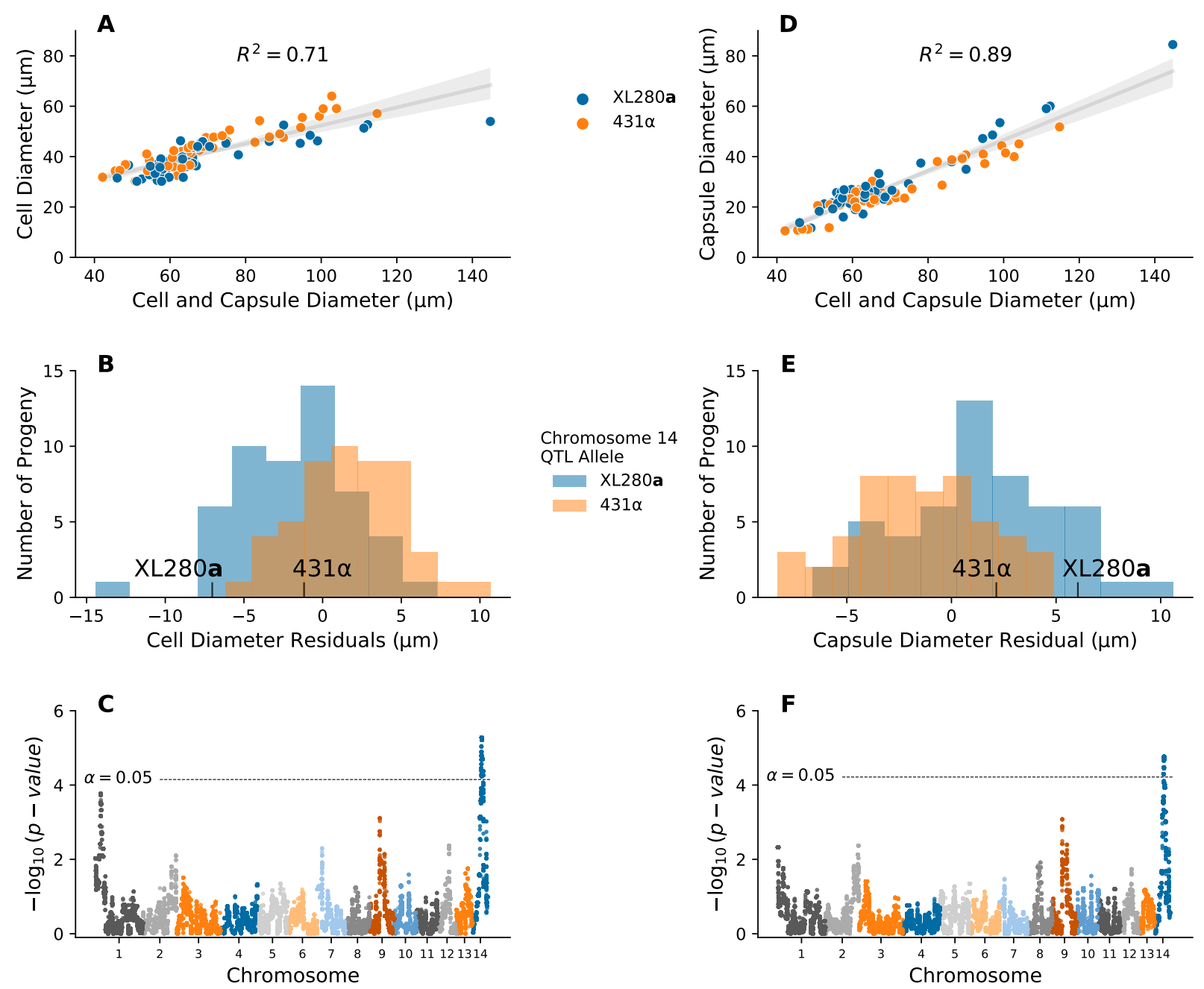

S2 Fig. QTL analysis of variation in cell and capsule size. A - C) Analysis of variation in cell diameter (y-axis) as a function of cell and capsule diameter ( $\mathrm{x}$-axis, $\mathbf{A}$ ), a histogram of the cell diameter residuals used in QTL mapping (B), and associated Manhattan plot (C). D - F) Analysis of variation in capsule diameter ( $y$-axis) as a function of cell and capsule diameter ( $\mathrm{x}$-axis, D), a histogram of the capsule diameter residuals used in QTL mapping (E), and temporaassociated Manhattan plot (F). Grey lines and shaded regions in $\mathbf{A}$ and $\mathbf{D}$ represent regression models and associated $95 \%$ confidence intervals. The variation explained by these models is annotated within each plot. For both cell and capsule diameter residuals a QTL is detected on chromosome 14. Dotted horizontal lines represent significance thresholds from permutation tests. Progeny cell and capsule diameter and cell and capsule diameter residual values are colored by the chromosome 14 QTL allele. 

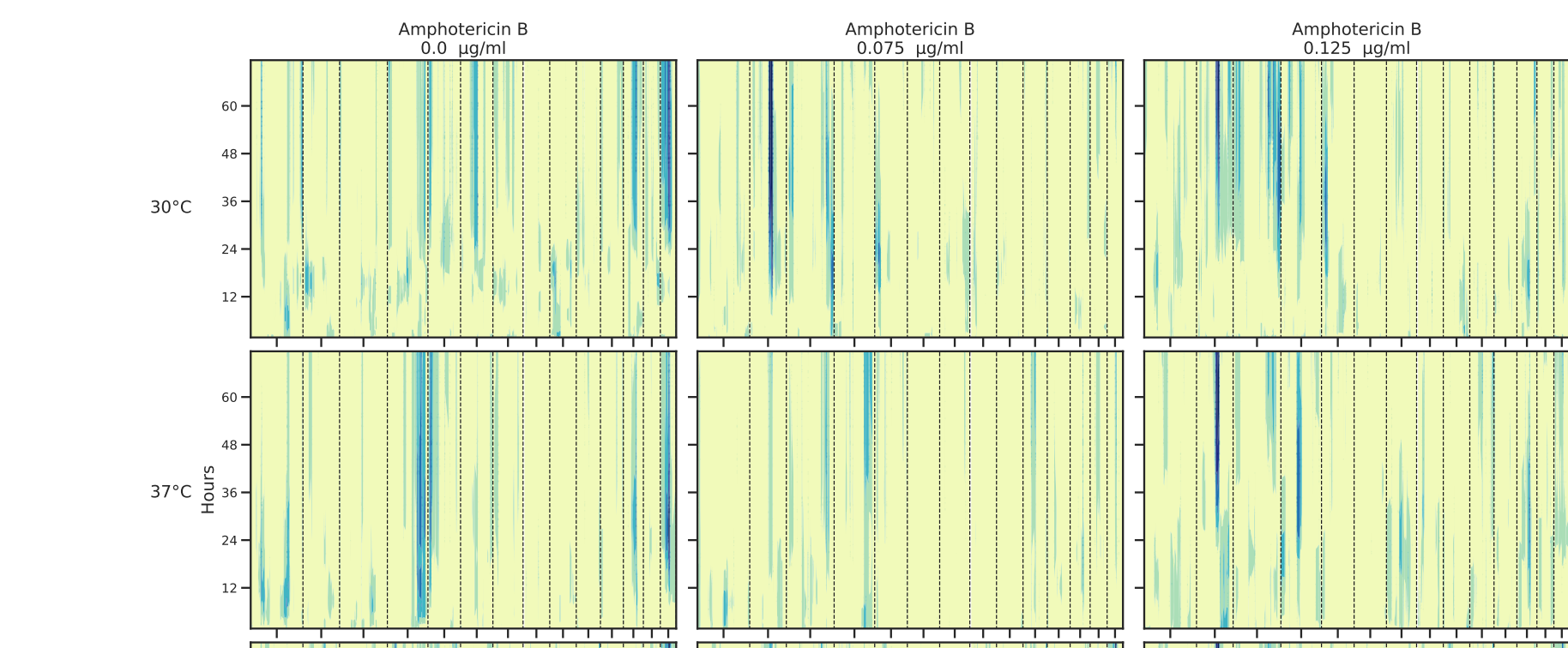

\&
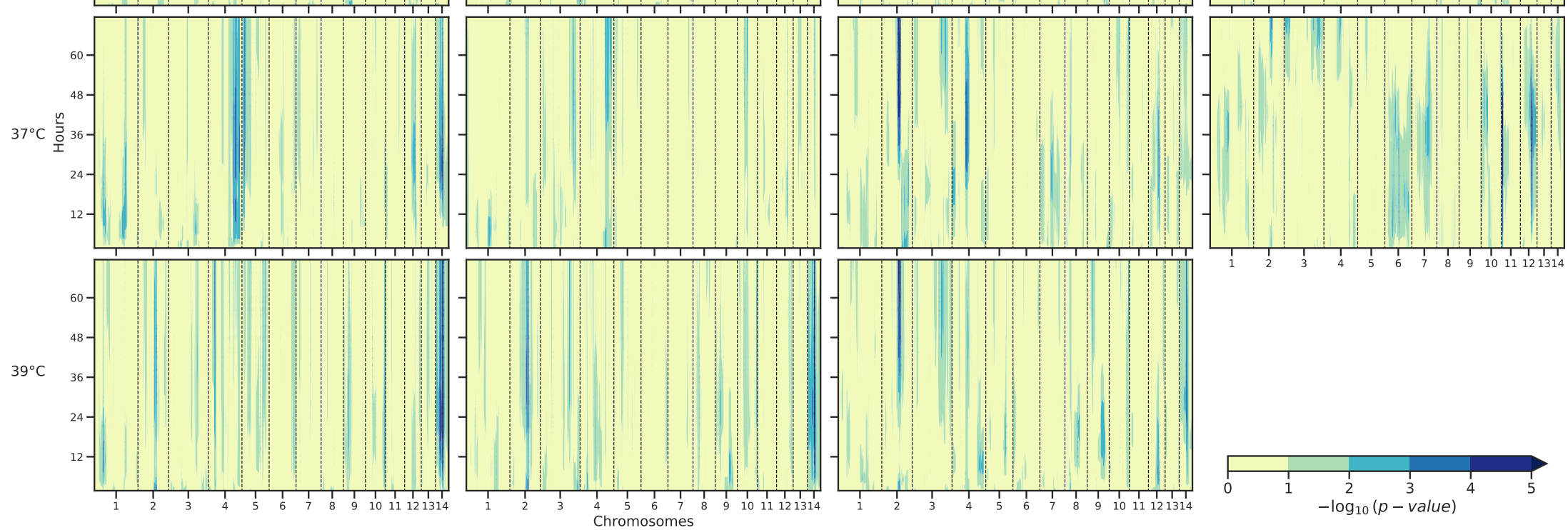

S3 Fig. Genome-wide temporal Manhattan plot. Genome-wide Manhattan heat maps of association between genotype and phenotype across 72 hour for combinations of temperature (rows) and amphotericin B (columns) concentrations in Fig 3. Across combinations of temperature and amphotericin B stress, the median growth AUC of segregants, calculated every 15 minutes for each 72 -hour time course, was regressed onto the parental genotypes of XL280a and $431 \alpha$. The yellow to blue colors depict the strength in association (as measured by the $-\log _{10}(p-$ value $)$ from the linear regression) between the growth AUC values and 3,108 bi-allelic haploblocks across segregants (x-axis) along the 72-hour time course (y-axis). 


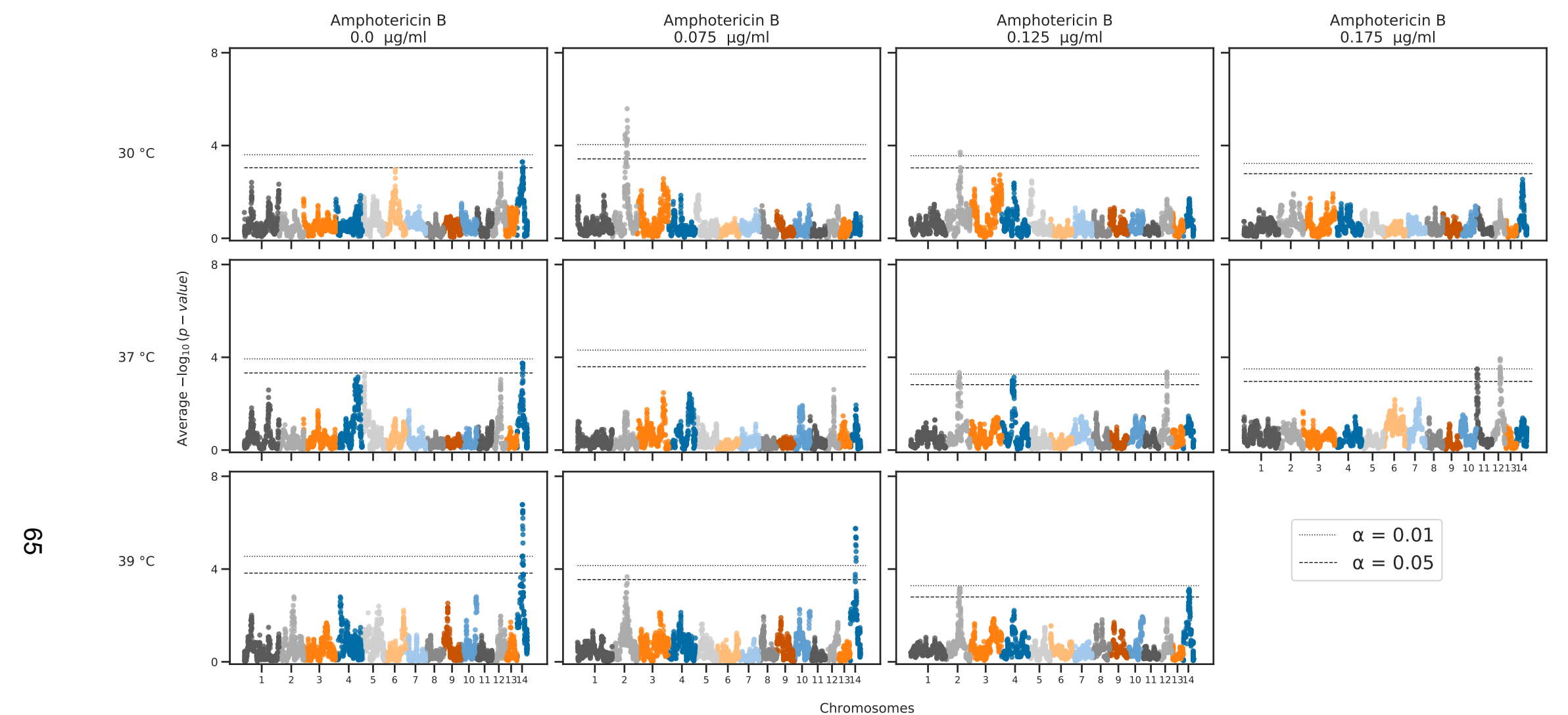

S4 Fig. Genome-wide temporal average Manhattan plot. Genome-wide Manhattan plots of average association across time between genotype and phenotype for combinations of temperature (rows) and amphotericin B (columns) stress. For each experimental condition in Fig 3, the median growth AUC of segregants across the 72-hour time course was regressed onto the parental genotypes of XL280a and $431 \alpha$. The $x$-axis represents positions along chromosomes (separated by colors) of 3,108 bi-allelic genetic variant sites, collapsed into haploblocks across segregants, and the y-axis is the average association across the 72-hour time course between genotype and the growth AUC values. Significance thresholds (horizontal dashed and dotted lines) were determined via permutation. 


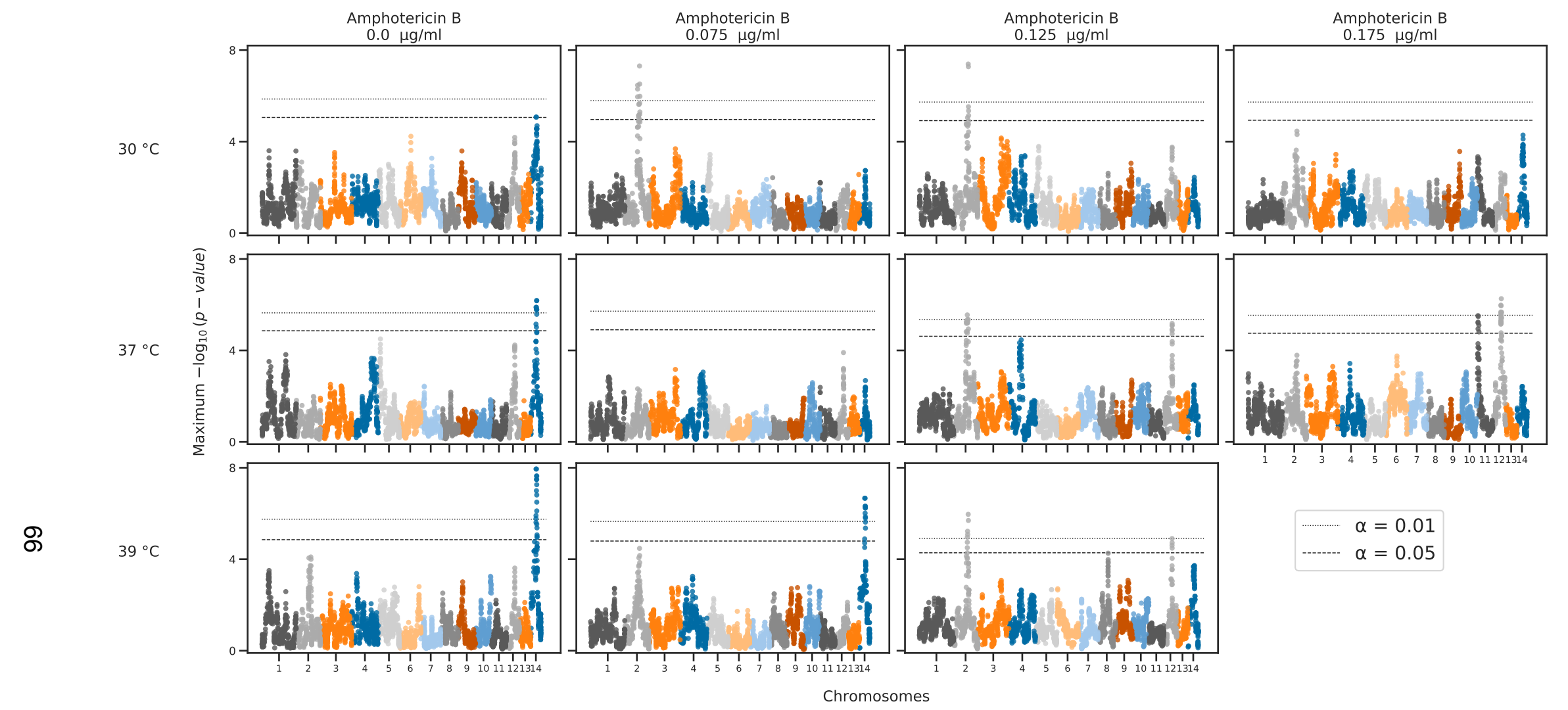

S5 Fig. Genome-wide temporal maximum Manhattan plot. Genome-wide Manhattan plots of maximum association across time between genotype and phenotype for combinations of temperature (rows) and amphotericin B (columns) stress. For each experimental condition in Fig 3, the median growth AUC of segregants across the 72-hour time course was regressed onto the parental genotypes of $\mathrm{XL280} a$ and $431 \alpha$. The $\mathrm{x}$-axis represents positions along chromosomes (separated by colors) of 3,108 , bi-allelic genetic variant sites, collapsed into haploblocks across segregants, and the y-axis is the maximum association across the 72-hour time course between genotype and the growth AUC values. Significance thresholds (horizontal dashed and dotted lines) were determined via permutation. 


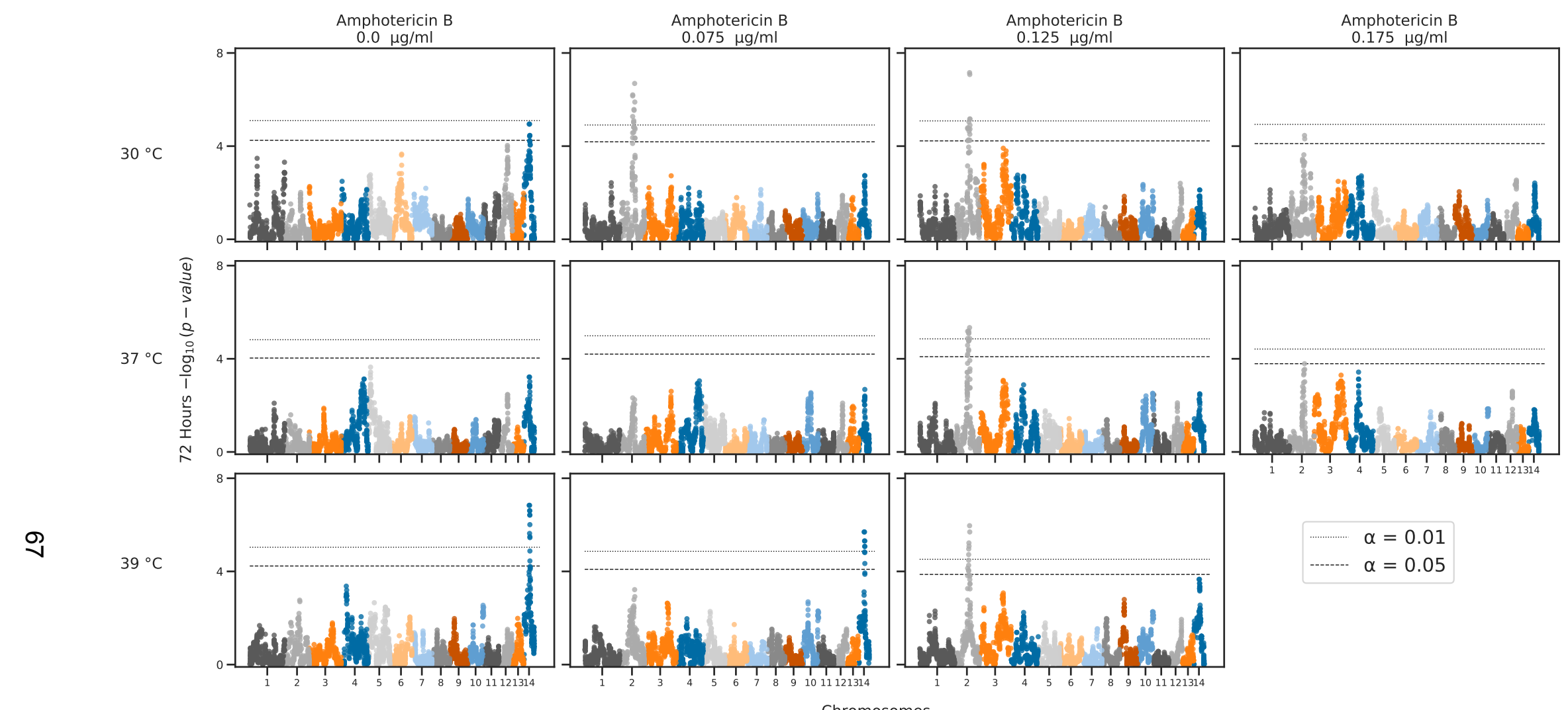

Chromosomes

S6 Fig. Genome-wide 72-hour Manhattan plot. Genome-wide Manhattan plots of association between genotype and phenotype for combinations of temperature (rows) and amphotericin B (columns) stress. For each experimental condition in Fig 3 , the median growth AUC of segregants at 72 hours of segregants was regressed onto the parental genotypes of XL280a and $431 \alpha$. The X-axis represents positions along chromosomes (separated by colors) of 3,108 bi-allelic genetic variant sites, collapsed into haploblocks across segregants and the $y$-axis is the association between genotype and the growth AUC values at 72 hour. Significance thresholds (horizontal dashed and dotted lines) were determined via permutation. 
$\mathbf{A}$

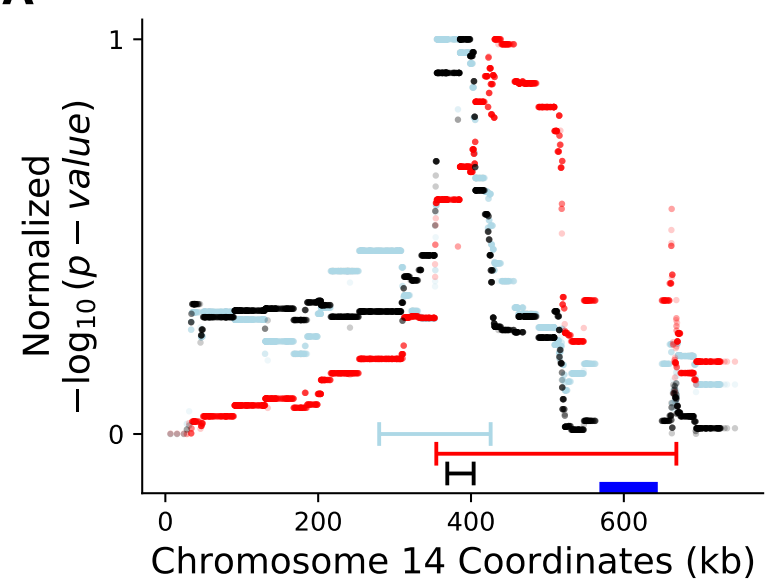

B

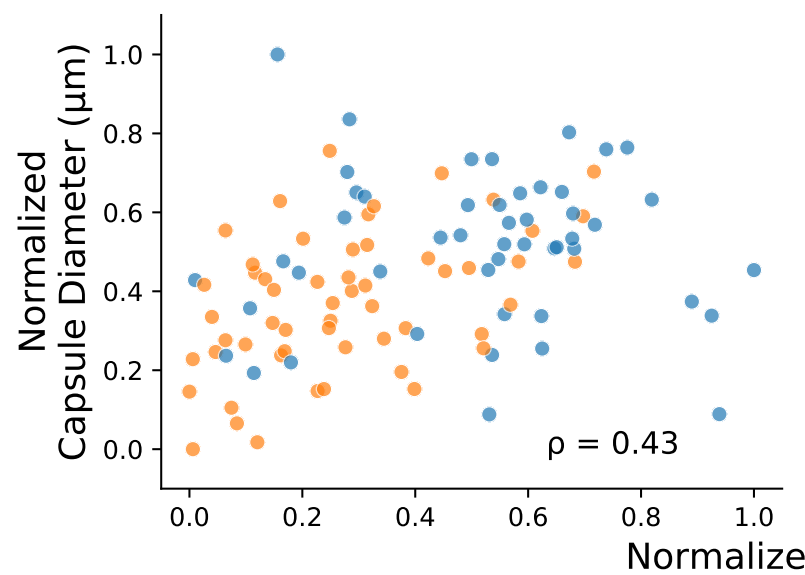

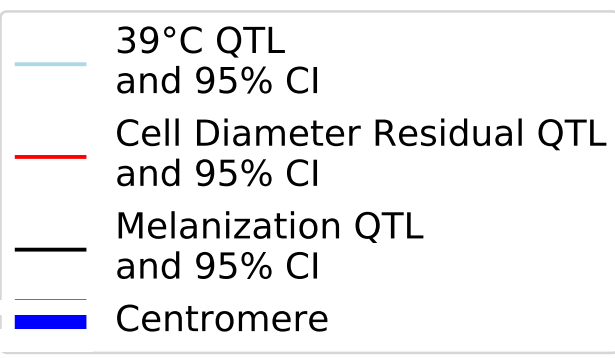
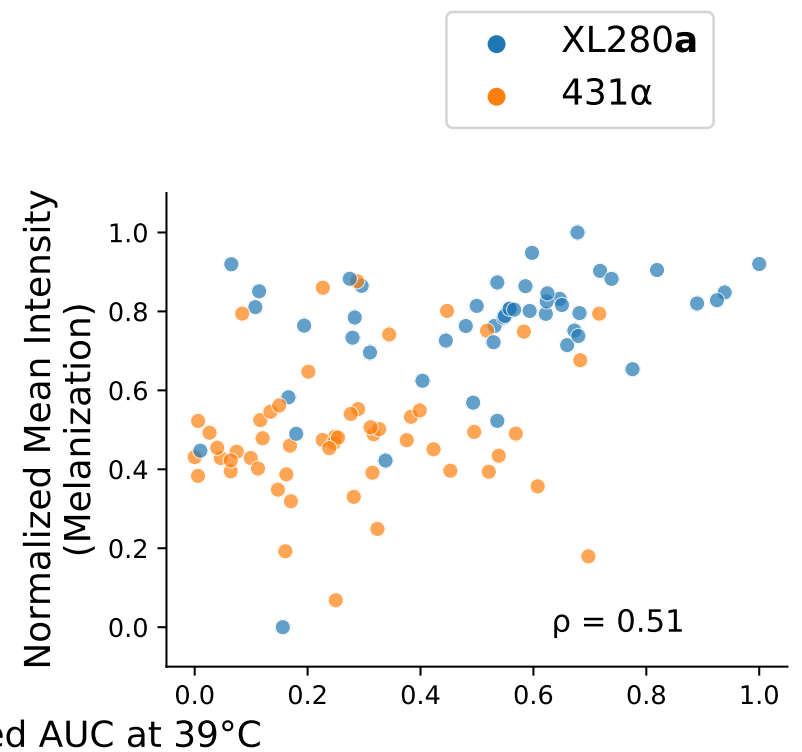

S7 Fig. Pleiotropic QTL along chromosome 14. A) The three QTL and associated confidence intervals $(\mathrm{Cl})$ for area under the curve at $39^{\circ} \mathrm{C}$ (light blue), cell diameter residuals (red), and melanization (black). Horizontal bar bells represent $95 \%$ confidence intervals. The location of the centromere on chromosome 14 is marked by a horizontal blue bar. B) Phenotypic relationships between capsule diameter and melanization ( $y$-axis of left and right panels, respectively) as a function of growth at $39^{\circ} \mathrm{C}$ (x-axis). Progeny values are colored by the allele at peak of the melanization QTL in A; blue for XL280a and orange for $431 \alpha$. The Spearman rank correlation $(\rho)$ between each pair of phenotypes is annotated within each plot. All QTL and phenotypic values in A and B (respectively) are re-scaled - in order to share the same scale - using max-min normalization. 


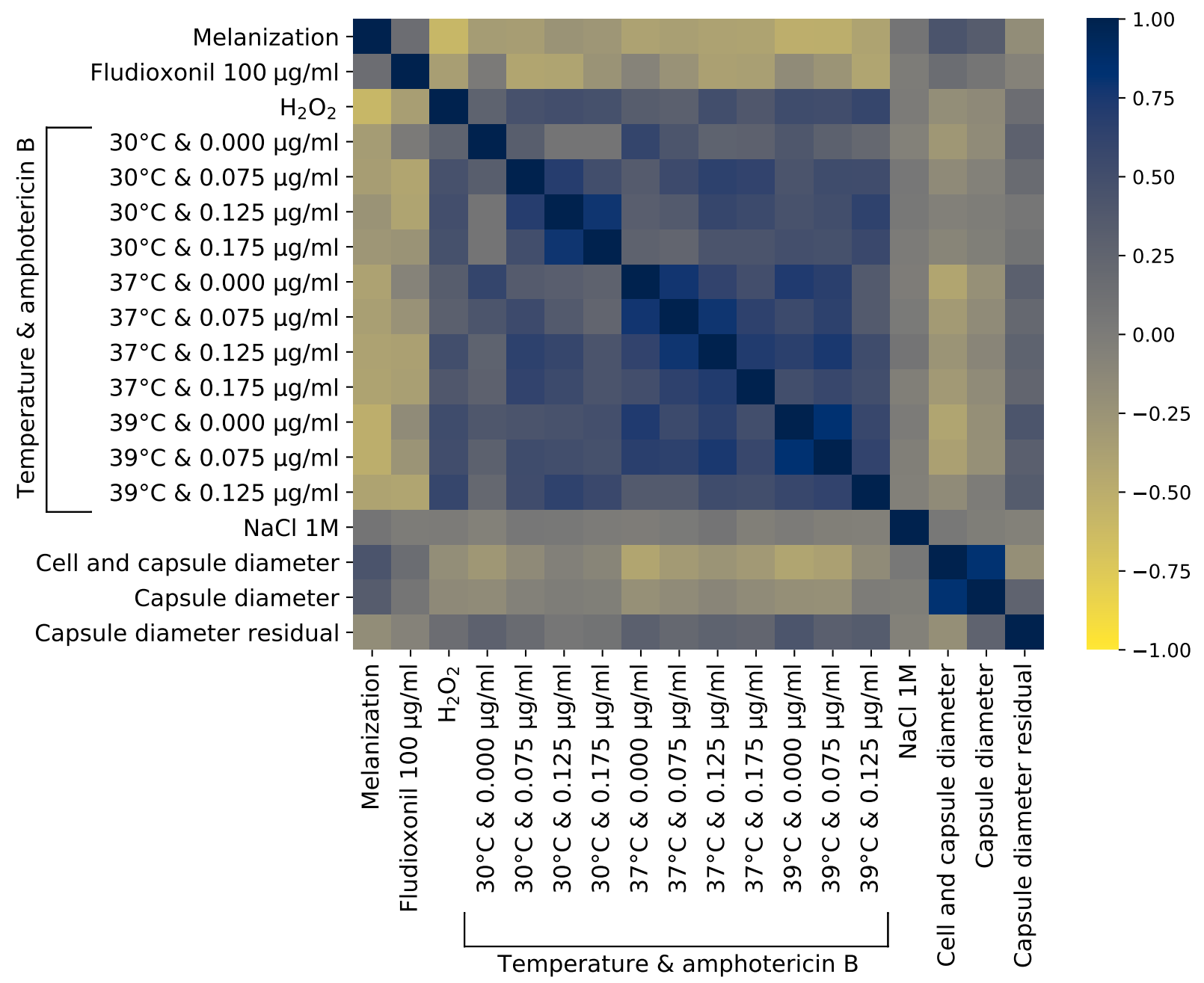

S8 Fig. Phenotypic correlations. Spearman rank correlations between C. deneoformans phenotypes. 

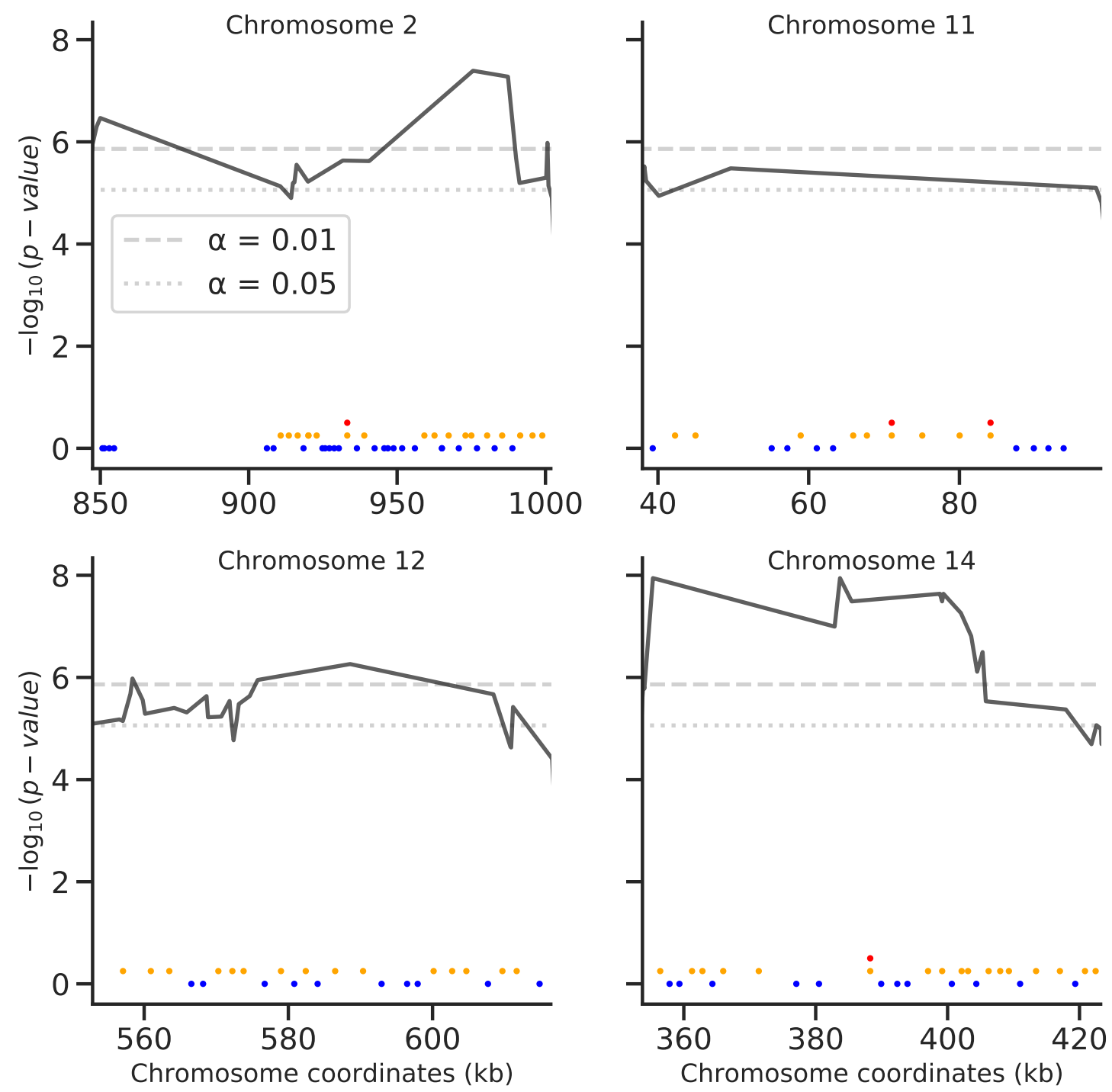

Chromosome coordinates $(\mathrm{kb})$

Predicted Changes
- Synonymous $\bullet$ Missense - Nonsense

S9 Fig. Changes in genes near QTL. Locations of genes, relative to the four identified QTL (black curves). From the JEC21 $\alpha$ reference genome [208], features were aligned to the XL280 $\alpha$ reference [137] and the changes and differences in protein sequence between XL280a, XL280 $\alpha$, and $431 \alpha$ were predicted. Dots along the $x$-axis represent location of mapped genes, colors indicate predicted change between the XL280a (or XL280 $\alpha$ ) and 431 $\alpha$ parental strains. 


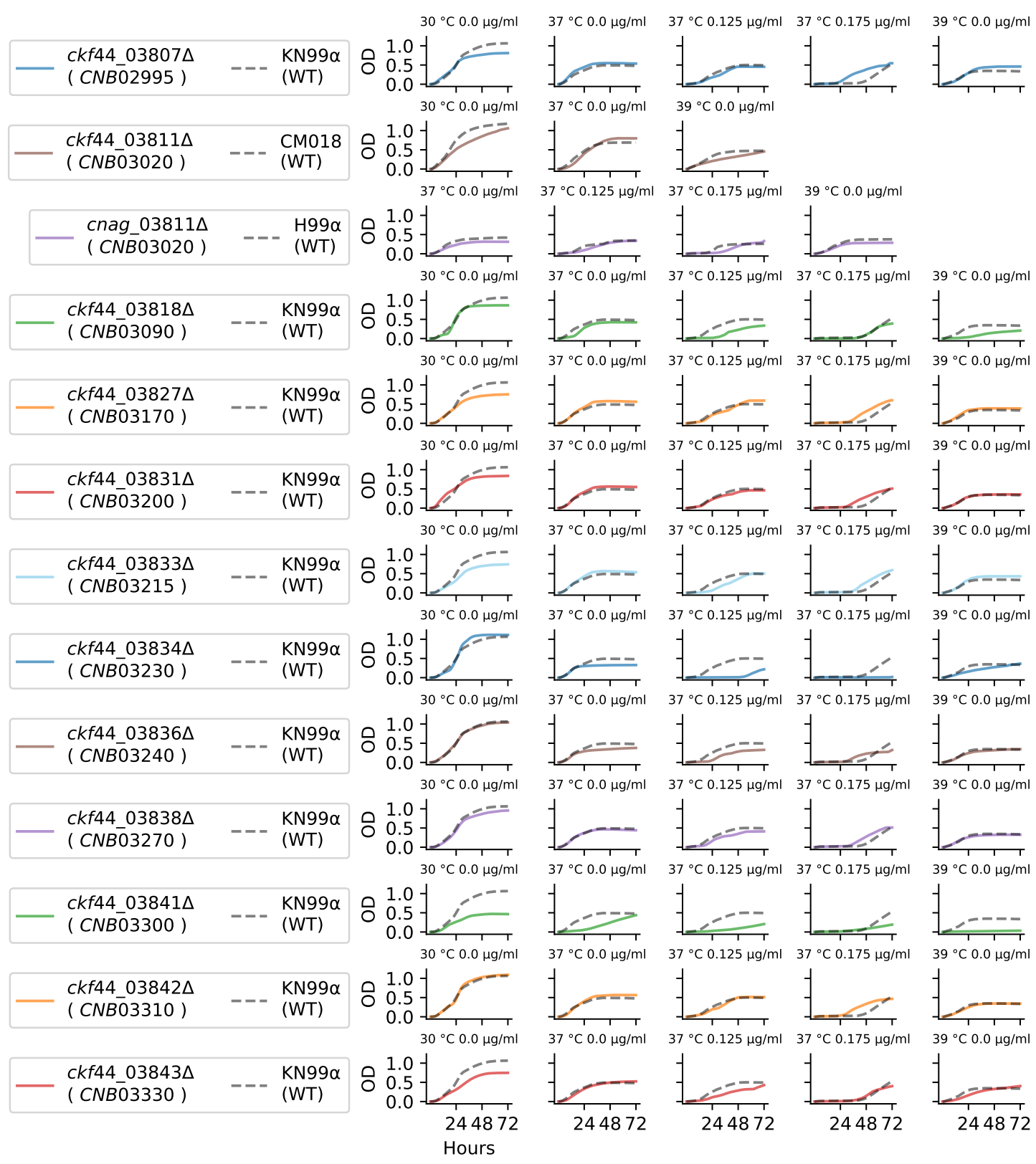

S10 Fig. Phenotypes of chromosome 2 candidate deletion mutants. Growth of candidate deletion mutant strains for genes within the QTL along chromosome 2. The available deletion mutants (rows, solid curves) of genes within the QTL and the corresponding wild type, C. neoformans strain, were assayed for growth in liquid culture for 72 hour at high temperatures $\left(30^{\circ}\right.$, $37^{\circ}$ and $39^{\circ} \mathrm{C}$ ) and in the presence of amphotericin B (at 0.125 and $0.175 \mu \mathrm{g} / \mathrm{ml}$ ). Legends on the far left show the gene names in the $C$. neoformans strain background with the corresponding C. deneoformans gene name. 


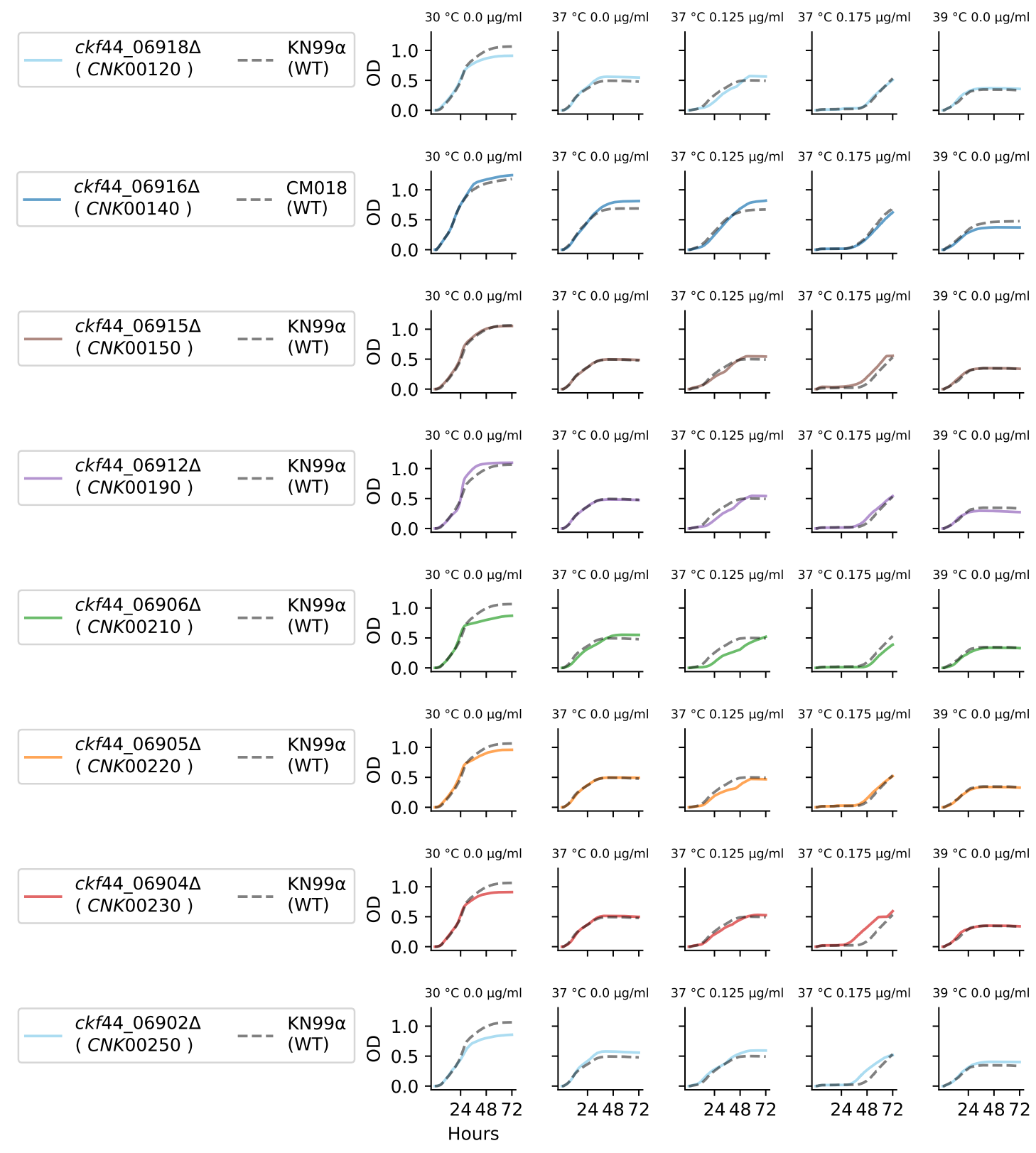

S11 Fig. Phenotypes of chromosome 11 candidate deletion mutants. Growth of candidate deletion mutant strains for genes within the QTL along chromosome 11. The available deletion mutants (rows, solid curves) of genes within the QTL and the corresponding wild type, C. neoformans strain, were assayed for growth in liquid culture for 72 hour at high temperatures $\left(30^{\circ}\right.$, $37^{\circ}$ and $39^{\circ} \mathrm{C}$ ) and in the presence of amphotericin B (at 0.125 and $0.175 \mu \mathrm{g} / \mathrm{ml}$ ). Legends on the far left show the gene names in the $C$. neoformans strain background with the corresponding C. deneoformans gene name. 


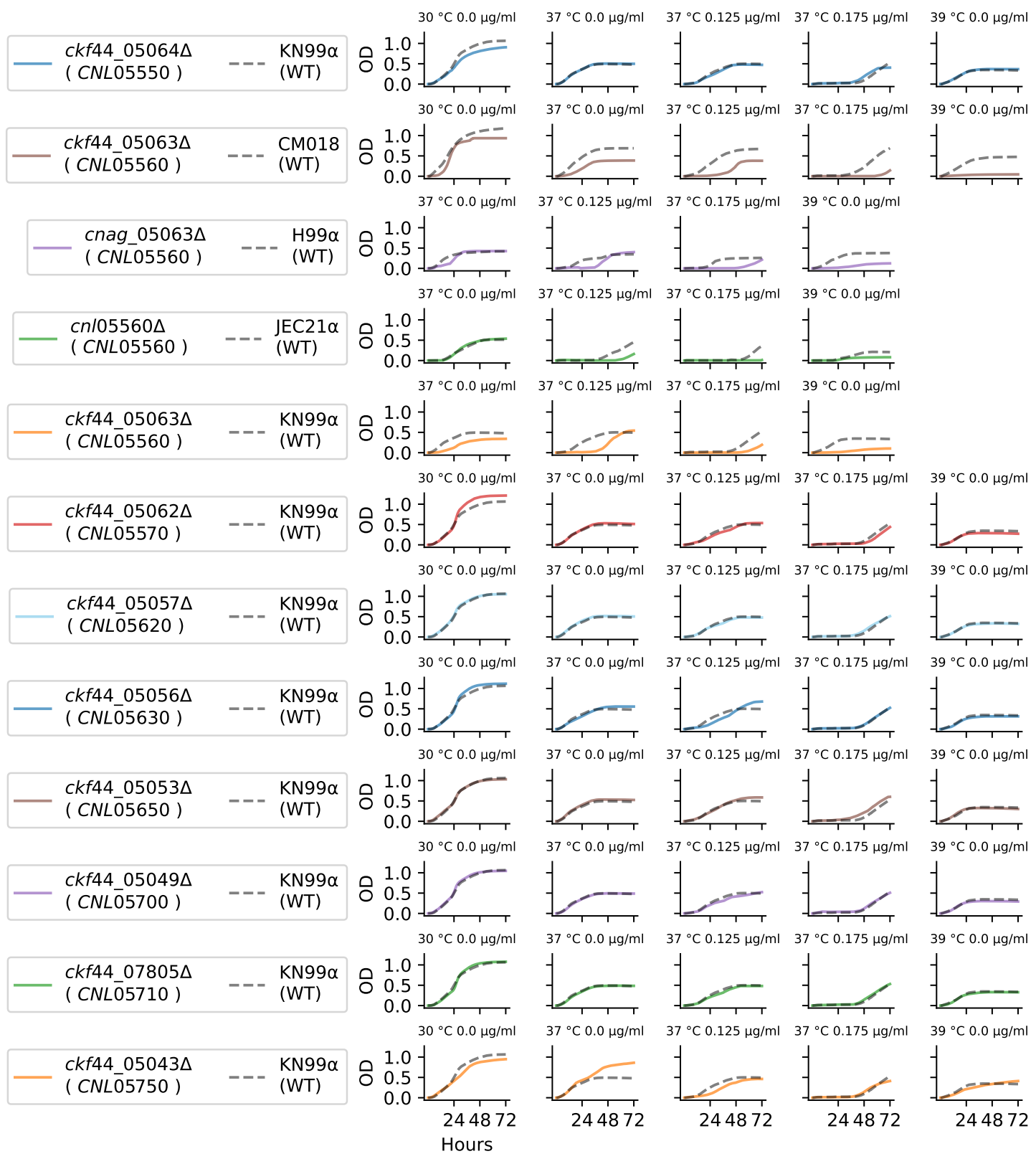

S12 Fig. Phenotypes of chromosome 12 candidate deltion mutants. Growth of candidate deletion mutant strains for genes within the QTL along chromosome 12. The available deletion mutants (rows, solid curves) of genes within the QTL and the corresponding wild type, C. neoformans strain, were assayed for growth in liquid culture for 72 hour at high temperatures $\left(30^{\circ}\right.$, $37^{\circ}$ and $39^{\circ} \mathrm{C}$ ) and in the presence of amphotericin B (at 0.125 and $0.175 \mu \mathrm{g} / \mathrm{ml}$ ). Legends on the far left show the gene names in the $C$. neoformans strain background with the corresponding C. deneoformans gene name. 


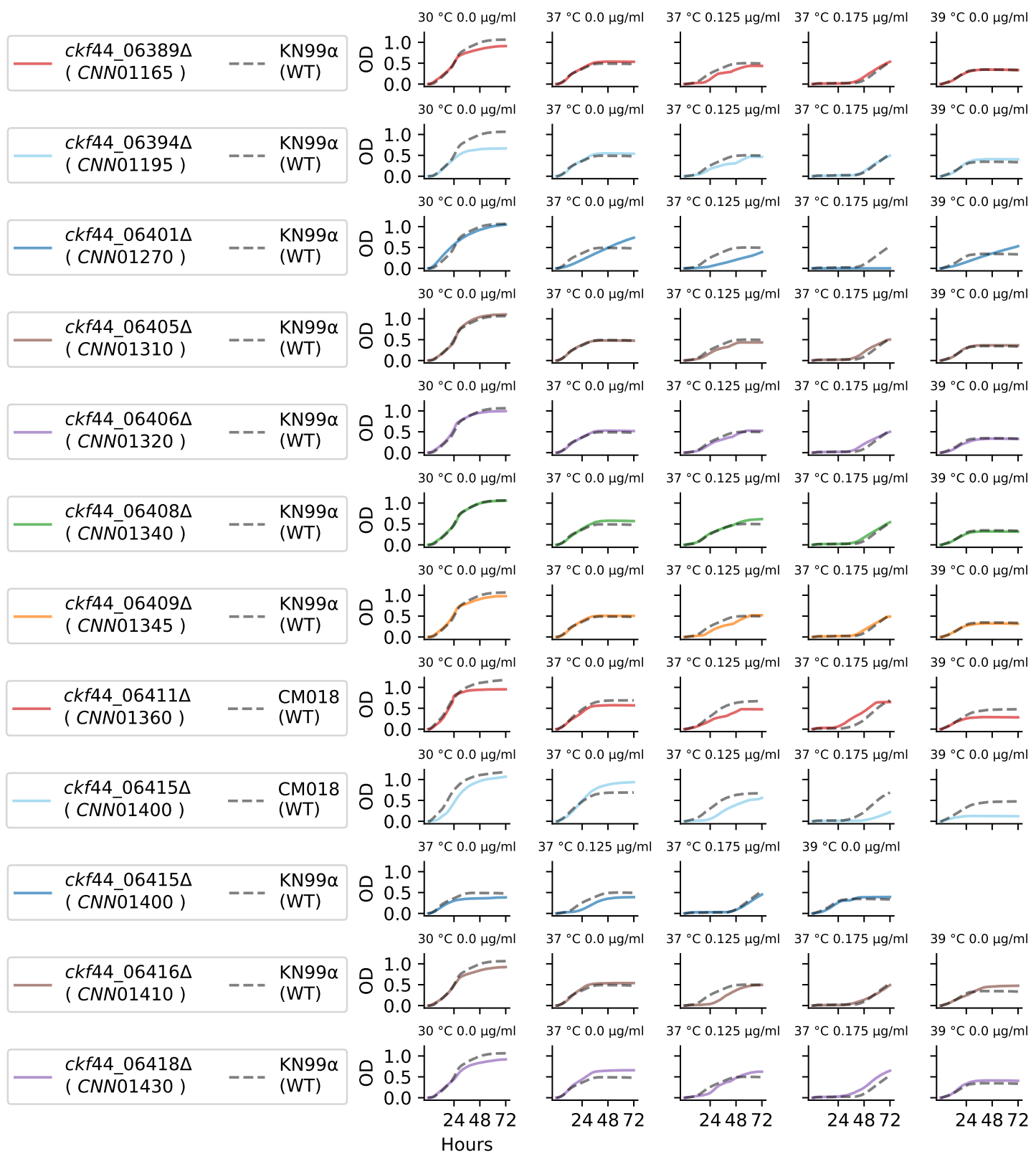

S13 Fig. Phenotypes of chromosome 14 candidate deltion mutants. Growth of candidate deletion mutant strains for genes within the QTL along chromosome 14. The available deletion mutants (rows, solid curves) of genes within the QTL and the corresponding wild type, C. neoformans strain, were assayed for growth in liquid culture for 72 hour at high temperatures $\left(30^{\circ}\right.$, $37^{\circ}$ and $39^{\circ} \mathrm{C}$ ) and in the presence of amphotericin B (at 0.125 and $0.175 \mu \mathrm{g} / \mathrm{ml}$ ). Legends on the far left show the gene names in the $C$. neoformans strain background with the corresponding C. deneoformans gene name. 


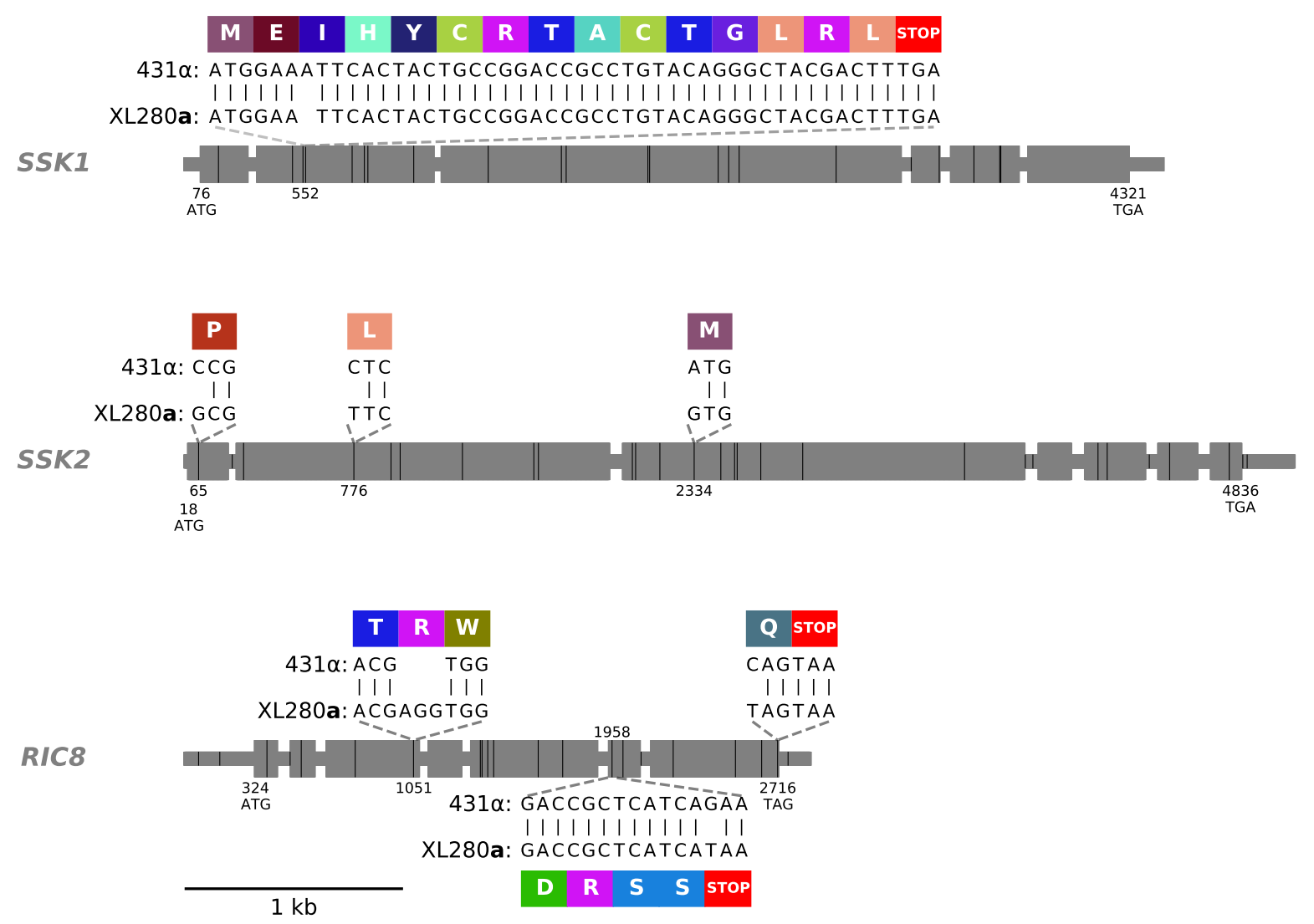

S14 Fig. SSK1, SSK2, and RIC8 gene models. Exons are shown as large grey rectangles, while the introns, 5' UTR, and 3' UTR are shown as grey, horizontal lines. The positions of bi-allelic genetic variants between the parental strains, $431 \alpha$ and XL280a are marked by black, vertical lines. The positions of the predicted start and stop codons are annotated along the bottom of the gene bodies. Within the second exon of SSK1, an insertion site of a single nucleotide, present in the $431 \alpha$ parental strain is annotated and this insertion is predicted to cause a frame shift that leads to a downstream early stop-gain. Within the first, second, and third exons of SSK2, three SNPs are annotated that lead to non-synonymous changes. The allelic states of the last two non-synonymous changes in SSK2 have been previously identified by [143]. Within the third and last exon of $R / C 8$, an in-frame codon deletion and shift in the predicted stop-codon (respectively) are seen in the $431 \alpha$ parental strain background. In the second to last exon of RIC8, a single-nucleotide polymorphism is present in the XL280a parental strain that is predicted to cause a premature stop. The local, predicted translations of the regions near these non-synonymous, genetic variants and associated amino acids are annotated in colored rectangles. 


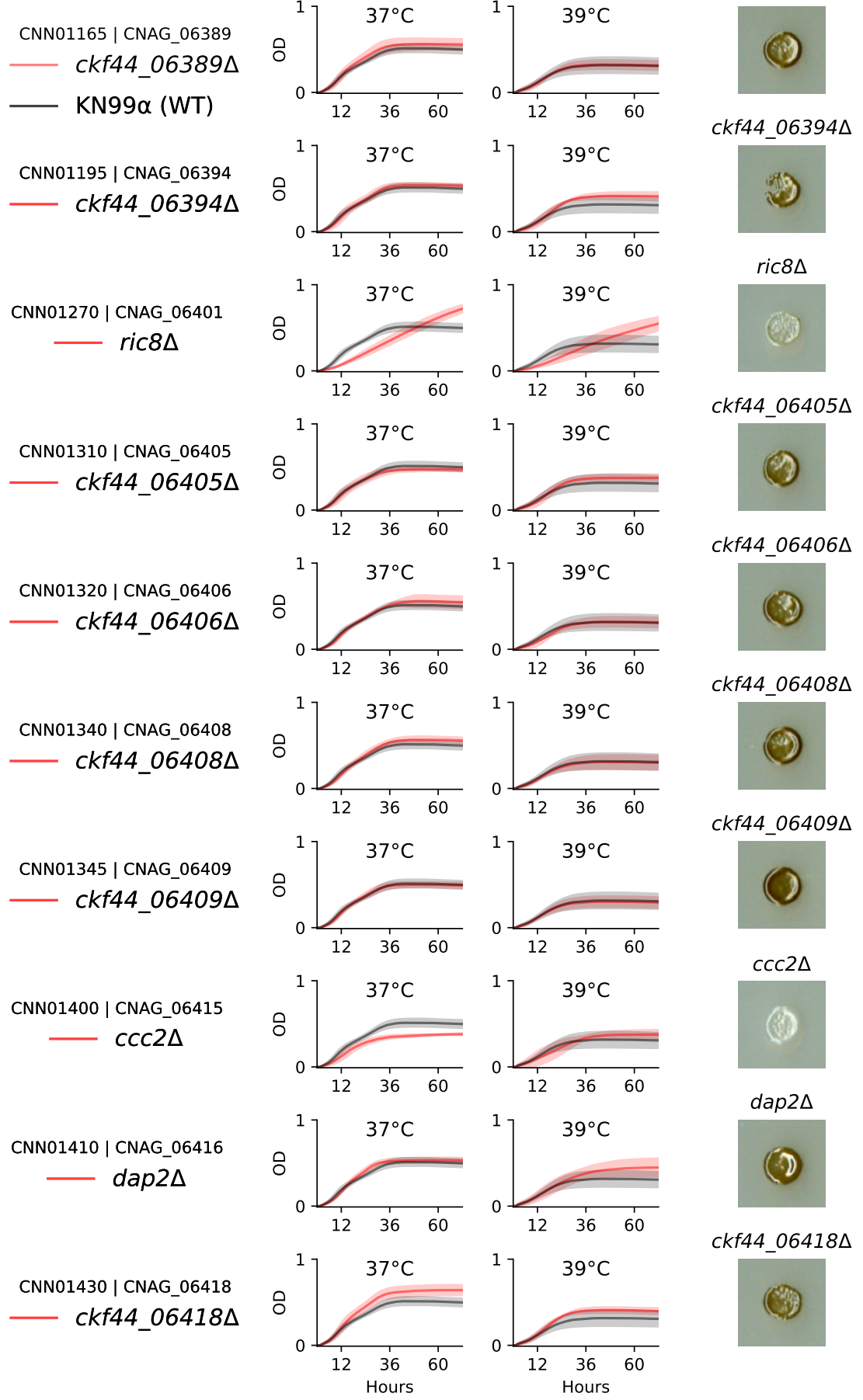

S15 Fig. High temperature growth and melanization phenotypes. High temperature growth and melanization phenotypes of chromosome 14 candidate quantitative trait genes. The available deletion strains (rows) in the KN99 $\alpha$ strain background of orthologous genes within the chromosome 14 QTL were assayed for high temperatures growth $\left(37^{\circ}\right.$ and $\left.39^{\circ} \mathrm{C}\right)$ in liquid culture and melanization on L-DOPA plates (columns, left to right respectively). Legends on the far left list the orthologous gene names in the C. deneoformans (JEC21 $\alpha$ ) and C. neoformans $(\mathrm{H} 99 \alpha)$ background. Red and black curves display mean high temperature growth for the deletion strain and KN99 $\alpha$ wild type (WT) strain (respectively) and shaded regions represent $95 \%$ confidence intervals. 

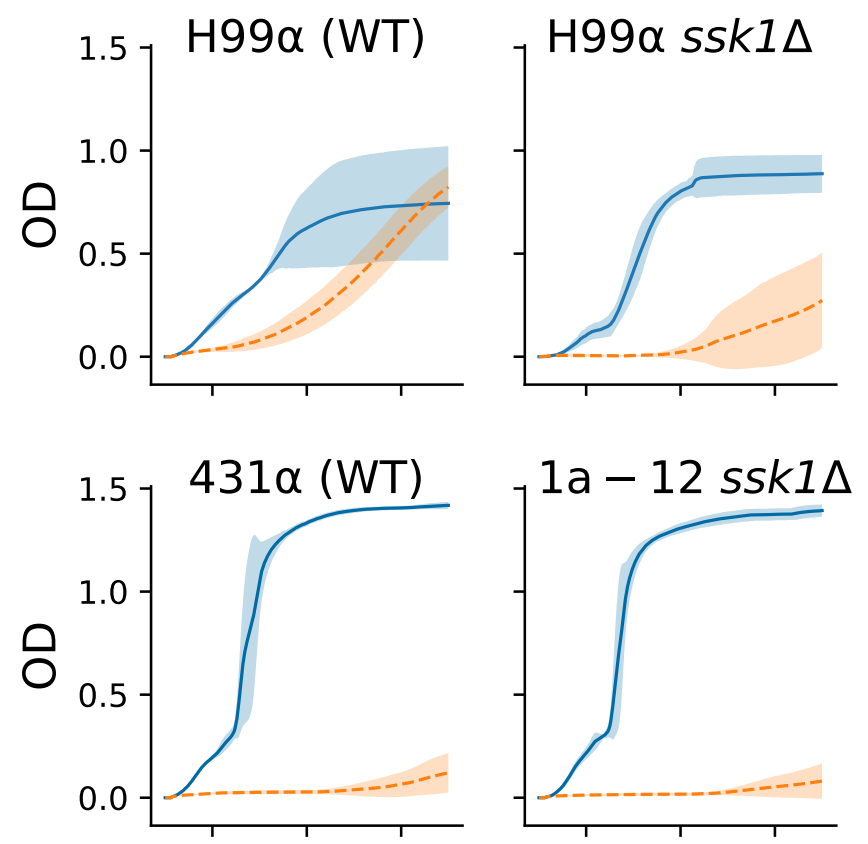

Amphotericin B $(\mu \mathrm{g} / \mathrm{ml})$
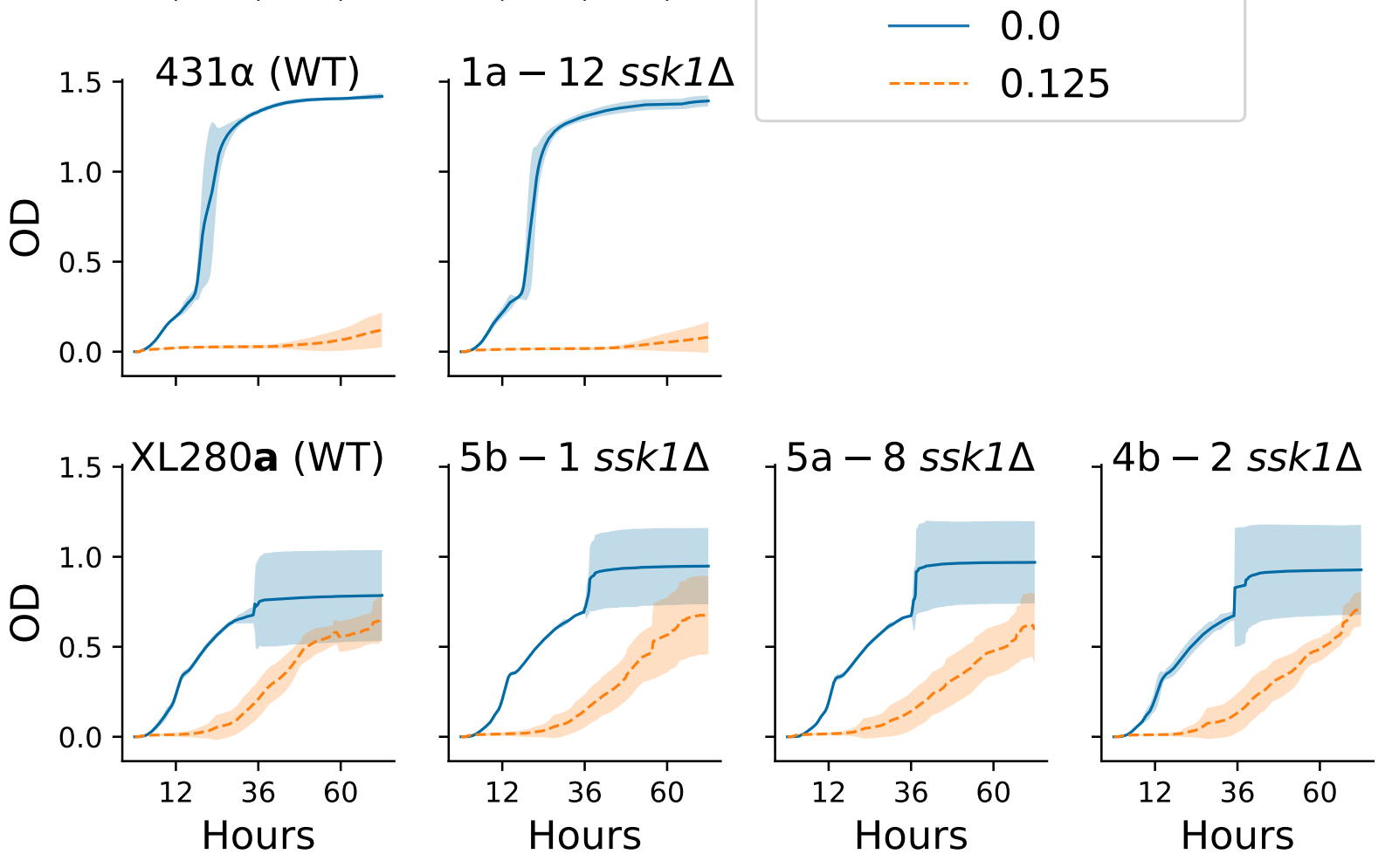

S16 Fig. C. neoformans and C. deneoformans ssk1 deletion mutant strain phenotypes. Growth of $C$. neoformans and $C$. deneoformans ssk1 deletion mutant strains. Across the rows and columns, the growth in liquid culture of wild type (WT) and ssk1 $\Delta$ strains (y-axis, optical density, $595 \mathrm{~nm}$ ) incubated for 72 hour (x-axis) at $30^{\circ} \mathrm{C}$ with and without amphotericin $\mathrm{B}(0.125 \mu \mathrm{g} / \mathrm{ml})$. Rows separate strain backgrounds. Growth curves are shown in the first column for the $C$. neoformans WT strain, $\mathrm{H} 99 \alpha$ (first row) and the C. deneoformans strains XL280a and $431 \alpha$ (last two rows, respectively). The growth curves of ssk1 $1 \Delta$ strains, per background, are depicted in the second, third, and fourth columns. Solid blue curves and dashed orange curves represent mean growth curves with 0.0 and $0.125 \mu \mathrm{g} / \mathrm{ml}$ of amphotericin B (respectively) and shaded regions are $95 \%$ confidence intervals. 


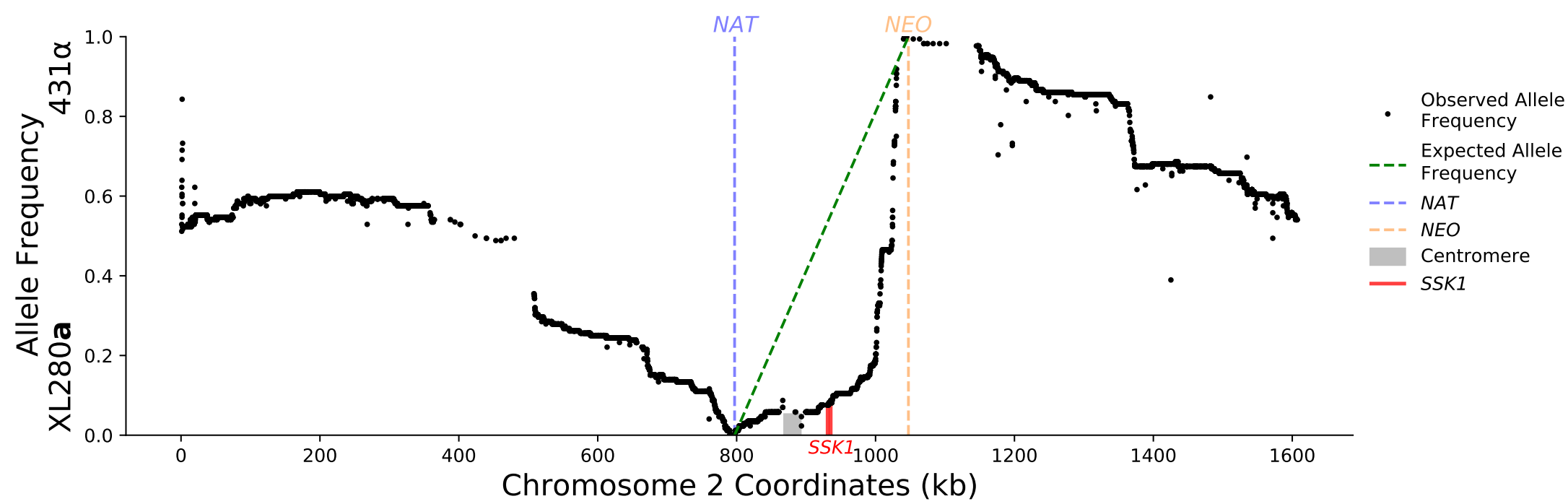

S17 Fig. Chromosome 2 allele frequencies. Allele frequency across bi-allelic, genetic variant sites of chromosome 2 . Across chromosome 2 (x-axis) for the progeny generated from fine mapping, the position and allele frequency (y-axis) of genetic variants between the parental strains XL280a and $431 \alpha$ are shown. The position of the selectable markers transformed within the parental backgrounds are shown by vertical, blue and orange, dashed lines. The expected allele frequency in this region given the marker locations is shown with a green, dashed line. The positions of the centromere and the SSK1 gene are shown by grey and red rectangles, respectively. 
$\mathbf{A}$
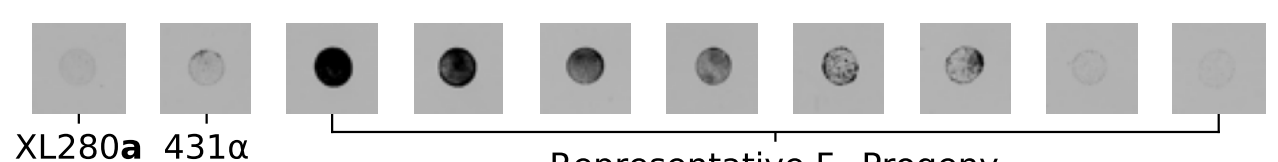

Representative $F_{1}$ Progeny

B

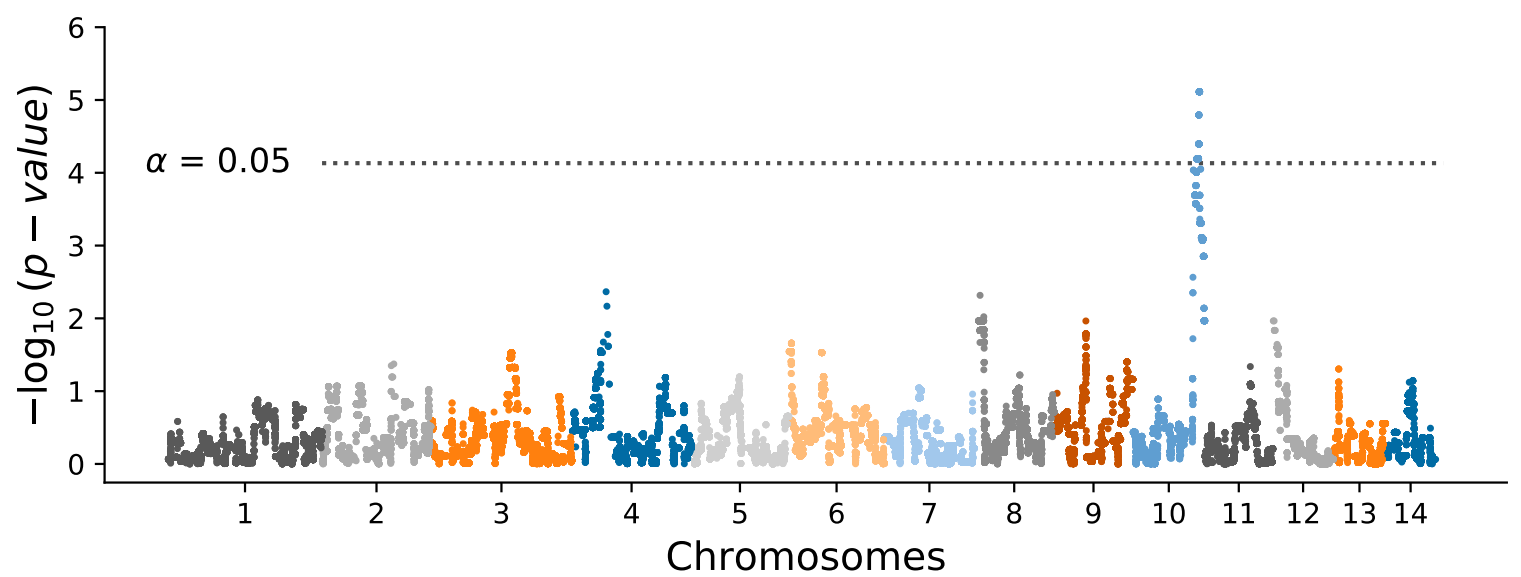

C

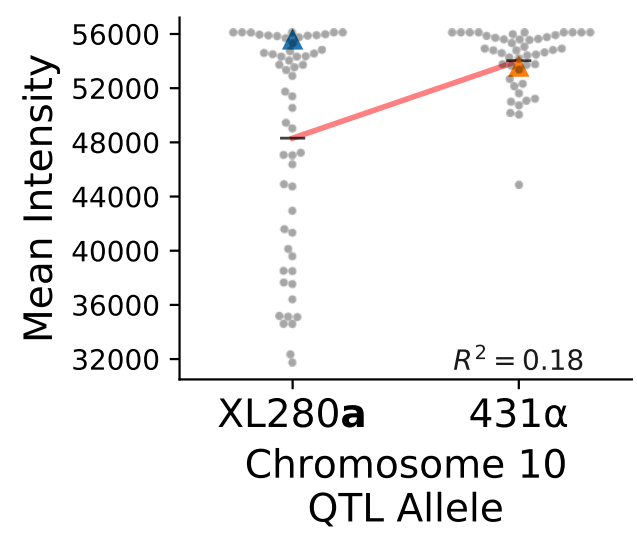

S18 Fig. Osmotic shock QTL. QTL analysis of variation in response to osmotic shock. A) Growth phenotypes of parental strains grown on media with $1 \mathrm{M} \mathrm{NaCl}$ and range of phenoytpes of their segregants. B) Manhattan plot of the association between genotype and growth in response to osmotic shock. The $x$-axis represents chromosomal locations of haploblocks and the $y$-axis represents the strength in association between genotype and variation in growth as measured by the mean intensity from translucent scans. C) Mean intensity (arbitrary units) of segregants (gray dots) from translucent scans as a function of allele at the peak of chromosome 10 QTL. The parental phenotypes are displayed by blue and orange triangles. Black horizontal lines denote the phenotypic means by allele and a red line represents a regression model relating genotype to phenotype. The heritablity - estimated from this regression model - is $\sim 18$ and annotated in black. 


\begin{tabular}{cccc}
\hline $\begin{array}{c}\text { SSK1 } \\
\text { Allele }\end{array}$ & $\begin{array}{c}\text { SSK2 } \\
\text { Allele }\end{array}$ & $\begin{array}{c}\text { \# of } \\
\text { Resistant }\end{array}$ & $\begin{array}{c}\text { \# of } \\
\text { Sensitive }\end{array}$ \\
\hline XL280a & XL280a & 20 & 0 \\
$431 \alpha$ & XL280a & 7 & 0 \\
XL280a & $431 \alpha$ & 10 & 130 \\
$431 \alpha$ & $431 \alpha$ & 6 & 0 \\
\hline
\end{tabular}

S19 Fig. Fludioxonil phenotypes of additional C. deneoformans progeny. The segregants represent the possible combinations of the SSK1 and SSK2 alleles from the XL280a strain, CF1730, and the $431 \alpha$ strains, CF1705, CF1706, CF1707. Within these progeny, of the 140 progeny with the SSK1 allele from XL280a parental strain and the SSK2 allele from the $431 \alpha$ parental strains (second to last row), 130 (93\%) demonstrated sensitivity to fludioxonil (100 $\mu \mathrm{g} / \mathrm{ml}$ ). All other combinations of the parental alleles in the fine-mapping progeny demonstrated resistance to fludioxonil. 
A Phenotype \& QTL Amphotericin $\mathrm{B}$ resistance $-=\left(37^{\circ} \mathrm{C}\right.$ and $\left.0.125 \mu \mathrm{g} / \mathrm{ml}\right)$

$-\mathrm{H}_{2} \mathrm{O}_{2}$ resistance

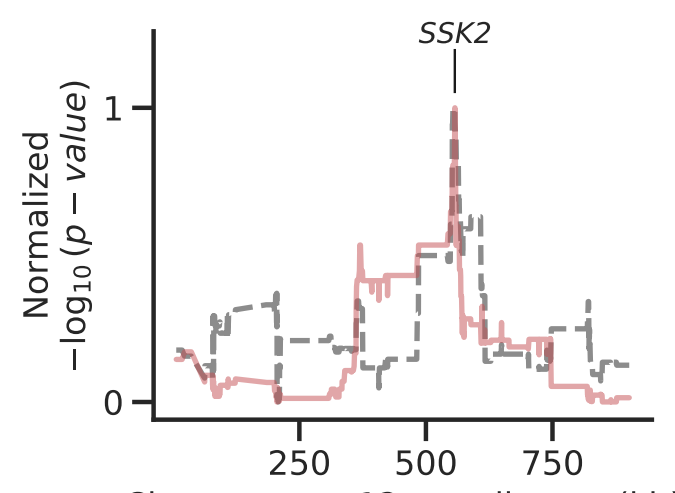

Chromosome 12 coordinates $(\mathrm{kb})$
B

Chromosome 12

QTL allele

- XL280a

- $431 \alpha$

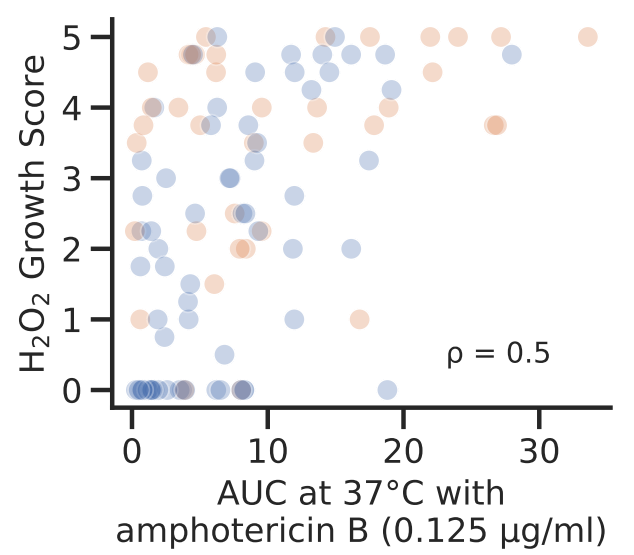

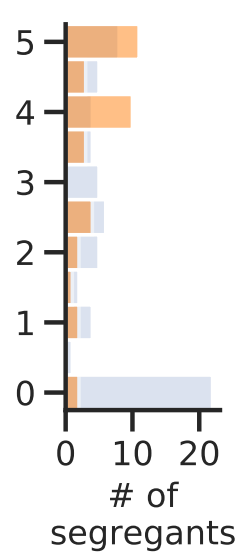

S20 Fig. Pleiotropic chromosome 12 QTL. Chromosome $12 \mathrm{QTL}$ and phenotypes for $\mathrm{H}_{2} \mathrm{O}_{2}$ and amphotericin $\mathrm{B}$ resistance. A) Normalized strength in association (y-axis) for the Chromosome 12 QTL for $\mathrm{H}_{2} \mathrm{O}_{2}$ (red) and amphotericin $\mathrm{B}$ resistance (black). The location of the candidate QTG, SSK2 is annotated. B) Median $\mathrm{H}_{2} \mathrm{O}_{2}$ growth score (y-axis) as a function of $A U C$ at $37^{\circ} \mathrm{C}$ with $0.125 \mu \mathrm{g} / \mathrm{ml}$ of amphotericin B (x-axis). The Spearman rank correlation is annotated within the plot. Segregant values are colored by their peak allele at chromosome 12 and a histogram in the right panel counts the number of segregants (x-axis) per $\mathrm{H}_{2} \mathrm{O}_{2}$ score. 
A

\begin{tabular}{l|ccc} 
SSK2 & XL280a & $431 \alpha$ & Total \\
RIC8 & & & \\
\hline XL280a & 23 & 26 & 49 \\
$431 \alpha$ & 42 & 13 & 55 \\
Total & 65 & 39 & 104
\end{tabular}

B

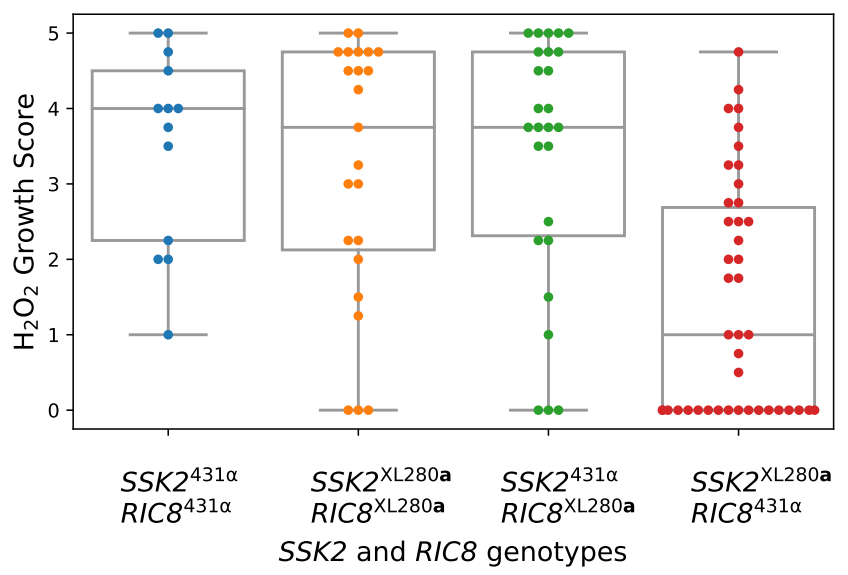

C
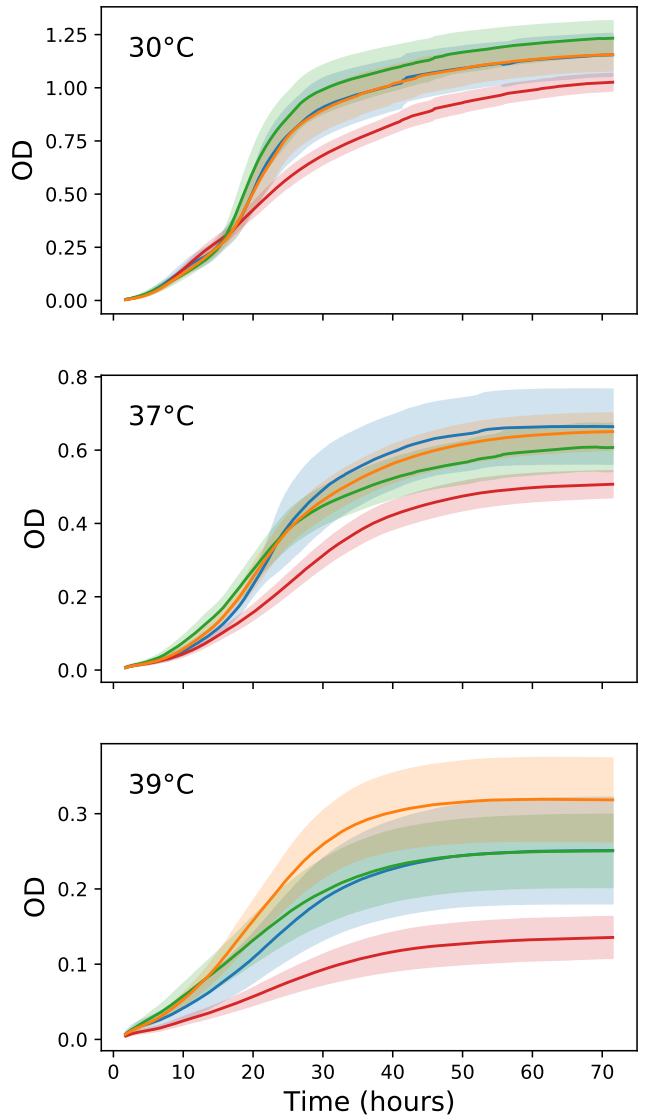

S21 Fig. Effect of SSK2 and RIC8 alleles on $\mathrm{H}_{2} \mathrm{O}_{2}$ and thermal tolerance. A) Contingency table of SSK2 (columns) and RIC8 (rows) alleles across segregants. B) Box- and swarm-plots of $\mathrm{H}_{2} \mathrm{O}_{2}$ growth scores (y-axis) by allelic combinations of SSK2 and RIC8 (x-axis). C) The mean growth curves (solid lines) and 95\% pointwise confidence intervals (shaded regions) per allelic combination of SSK2 and RIC8 across temperatures. OD is optical density sampled at $595 \mathrm{~nm}$. In panels $\mathbf{B}$ and $\mathbf{C}$, phenotypes are color coded by the combinations of SSK2 and RIC8 alleles listed in $\mathbf{A}$. 